\title{
Geodesic Flows on Manifolds of Negative Curvature with Smooth Horospheric Foliations
}

\author{
Thesis by \\ Renato Feres \\ In Partial Fulfillment of the Requirements \\ for the Degree of Doctor of Philosophy
}

California Institute of Technology 253-37

Pasadena, CA 91125

1989

(Submitted May 10, 1989) 


\section{Acknowledgment}

It is a pleasure to express my deep gratitude to Anatoly Katok - my research advisor and coauthor of [FK1] and [FK2] - for his vigorous guidance and generosity in sharing his time and ideas. I owe to him my taste for Dynamics, Ergodic Theory, and Russian cuisine.

I wish to thank Masahiko Kanai for helpful conversations and foremost for having provided the stuff upon which this work is based. I also owe to Livio Flaminio many delightful hours of intense discussion and to my friend and officemate Boris Hasselblatt his patience in helping me solve the mysteries of the word processor.

I am indebted to the Math department of Caltech for providing a stimulating work environment and financial support and to the A. P. Sloan Foundation for the financial support during the academic year of $1988 / 89$. 


\begin{abstract}
We improve a result due to $\mathrm{M}$. Kanai on the rigidity of geodesic flows on closed Riemannian manifolds of negative curvature whose stable or unstable (horospheric) foliation is smooth. More precisely, the main result proven here is: Let $M$ be a closed $\mathrm{C}^{\infty}$ Riemannian manifold of negative sectional curvature. Assume the stable or unstable foliation of the geodesic flow $\varphi_{t}: \mathrm{V} \rightarrow \mathrm{V}$ on the unit tangent bundle $\mathrm{V}$ of $\mathrm{M}$ is $\mathrm{C}^{\infty}$. Assume moreover that either (a) the sectional curvature of $\mathrm{M}$ satisfies $-4<\mathrm{K} \leq-1$ or (b) the dimension of $M$ is odd. Then the geodesic flow of $M$ is $C^{\infty}$-isomorphic (i. e., conjugate under a $\mathrm{C}^{\infty}$ diffeomorphism between the unit tangent bundles) to the geodesic flow on a closed Riemannian manifold of constant negative curvature.
\end{abstract}




\section{TABLE OF CONTENTS}

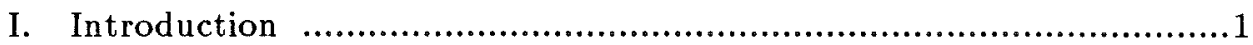

II. Definitions, Notations and Basic Facts ....................................

III. An Invariant Affine Connection on V ........................................ 18

IV. An Outline of Kanai's Work ....................................................225

V. Some Smooth Ergodic Theory .................................................... 30

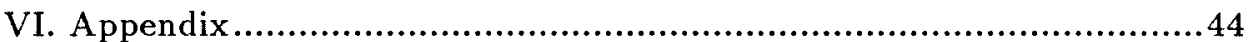




\section{Introduction}

On a compact $\mathrm{C}^{\infty}$ manifold $\mathrm{V}$, let us consider a $\mathrm{C}^{\infty}$ flow $\varphi_{t}: \mathrm{V} \rightarrow \mathrm{V}$. To simplify the notation we may, at times, denote the flow by $\varphi$.

$\varphi$ is an Anosov flow if for a given Riemannian norm $\|\cdot\|$ on $\mathrm{TV}$ the tangent bundle of $\mathrm{V}$ decomposes as a direct sum of continuous flow-invariant subbundles:

$$
\mathrm{TV}=\mathrm{E}^{+} \oplus \mathrm{E}^{-} \oplus \mathrm{E}^{0}
$$

( $\oplus$ denotes the Whitney sum of vector bundles) where for each $v \in V, E^{0}$ is the direction spanned by

$$
\dot{\varphi}(\mathrm{v})=\left.\frac{\mathrm{d}}{\mathrm{dt}}\right|_{t=0} \varphi_{t}(\mathrm{v})
$$

which is assumed to be everywhere nonzero, and there are constants $a>0$ and $b \geq 1$ such that for all $\mathrm{t} \geq 0$,

$$
\begin{gathered}
\left\|\left.\mathrm{D} \varphi_{-t}\right|_{\mathrm{E}^{+}}\right\| \leq \mathrm{b} \cdot \mathrm{e}^{-a t} \\
\left\|\left.\mathrm{D} \varphi_{t}\right|_{\mathrm{E}^{-}}\right\| \leq \mathrm{b} \cdot \mathrm{e}^{-a t}
\end{gathered}
$$

(see $[\mathrm{A}] \cdot$ )

Consider the distributions of linear subspaces of TV:

$\mathrm{E}^{+}$(resp. $\mathrm{E}^{+0}=\mathrm{E}^{+} \oplus \mathrm{E}^{0}$ ) - the strong (resp. weak) unstable distribution,

$\mathrm{E}^{-}$(resp. $\mathrm{E}^{-0}=\mathrm{E}^{+} \oplus \mathrm{E}^{0}$ ) - the strong (resp. weak) stable distribution.

These distributions are continuous and integrable. Denote the corresponding foliations by $\mathrm{W}^{+}\left(\mathrm{W}^{+0}\right)$ and $\mathrm{W}^{-}\left(\mathrm{W}^{-0}\right)$. We will refer to these foliations as the Anosov foliations of $\varphi$ and to the decomposition (1) as the Anosov splitting of TV.

A natural example of an Anosov flow is the geodesic flows on Riemannian manifolds of negative curvature. More precisely, the following proposition holds:

Proposition 1. (see, e.g., [Kl]) Let $\varphi_{t}: V \rightarrow V, t \in \mathbb{R}$, be the geodesic flow on the 
unit tangent bundle $V$ of a compact, $C^{\infty}$ Riemannian manifold $M$ of dimension $n$ and let $\|\cdot\|$ be a Riemannian norm on $V$. Let us suppose the existence of positive constants $k_{0}$ and $k_{1}$ such that for any two-plane $\alpha$ tangent to $M$, the sectional curvature $K(\alpha)$ of $\alpha$ satisfies

$$
-k_{0}^{2} \leq K(\alpha) \leq-k_{1}^{2}
$$

Then there exists a continuous, $\varphi$-invariant decomposition

$$
T V=E^{+} \oplus E^{-} \oplus E^{0}
$$

where $E^{0}$ is spanned by the geodesic spray $\dot{\varphi}$ and a constant $c \geq 1$ such that for all $t \geq 0$,

$$
\begin{gathered}
c^{-1} \cdot e^{-k_{0} t} \leq\left\|\left.D \varphi_{t}\right|_{E^{-}}\right\| \leq c \cdot e^{-k_{1} t} \\
c \cdot e^{k_{0} t} \geq\left\|\left.D \varphi_{t}\right|_{E^{+}}\right\| \geq c^{-1} \cdot e^{k_{1} t} .
\end{gathered}
$$

One basic question regarding Anosov flows in general and geodesic flows on manifolds of negative curvature in particular is to understand the regularity properties of the Anosov foliations (or the horospheric foliations, in the latter case).

In general, one should not expect the stable and unstable foliations of an Anosov flow to be very regular. It was proven by Anosov that they are always Hölder continuous but may fail to be $C^{1}$, even if the flow itself is $C^{\infty}$.

The following theorem of $\mathrm{B}$. Hasselblatt $[\mathrm{H}]$ justifies this claim. Recall that, given a periodic point $\mathrm{p}$ of a flow $\varphi$ and a hypersurface through $\mathrm{p}$ transverse to the flow, one can define the first return map - the map which assigns, to each point $\mathrm{p}^{\prime}$ near $\mathrm{p}$ that is also contained in the hypersurface, the next point in the forward orbit of $\mathrm{p}^{\prime}$ that intersects that same hypersurface.

Definition. Let $\mathfrak{S}$ denote the set of Anosov flows on a compact Riemannian manifold $\mathrm{V}$, equipped with an invariant symplectic form on $\mathrm{E}=\mathrm{E}^{+} \oplus \mathrm{E}^{-}$and give $\mathcal{S}$ the $\mathrm{C}^{\infty}$-topology. For $\varphi \in \mathcal{S}$, a periodic orbit $\mathrm{p}$ is called $\beta$-spread if the differential $\mathrm{D} \Phi$ of 
the return map $\Phi$ to a hypersurface through $p$ transverse to the flow has eigenvalues $\lambda_{1}$ and $\lambda_{2}$ satisfying $1<\left|\lambda_{2}\right|^{\beta}<\left|\lambda_{1}\right|$. Define

$$
\mathcal{S}_{\beta}=\{\varphi \in \mathcal{S}: \varphi \text { has a } \beta \text {-spread periodic orbit }\} \text {. }
$$

Theorem ([H]). For $\beta>1$, flows in $\mathfrak{S}_{\beta}$ generically do not have unstable distribution with Hölder exponent $2 / \beta$. ( If $2 / \beta \geq 1$, this means $C^{1+x}$ for $x=2 / \beta-1$.)

It can be shown as a consequence that in an open dense set of $\mathfrak{S}$, the Anosov foliations are not $\mathrm{C}^{2}$.

For a $\mathrm{C}^{\infty}$ Anosov flow on a three-dimensional, closed Riemannian manifold $\mathrm{M}$ preserving a smooth volume form, it was shown by S. Hurder and A. Katok [HK] that the Anosov foliations are always of class $\mathrm{C}^{1, \alpha}$, for $\alpha(\mathrm{x})=\mathrm{x} \log \mathrm{x}$ and if they are $\mathrm{C}^{2}$ it follows that they are, in fact, $\mathrm{C}^{\infty}$. In the case of geodesic flows on surfaces of negative curvature the last conclusion implies constant curvature (see [G]).

The only known examples of geodesic flows on Riemannian manifolds of negative curvature that have $\mathrm{C}^{2}$ Anosov foliations are the geodesic flows on Riemannian locally symmetric spaces of $\mathbb{R}$-rank 1 and negative curvature. These spaces are locally isometric to the hyperbolic spaces $\mathbb{R H}^{n}, \mathbb{C H}^{n}, \mathbb{H H}^{n}$, and $\mathbb{C a H}^{2}([\mathrm{Ht}])$. In these examples the Anosov foliations are, in fact, $C^{\omega}$.

In view of these facts, it makes sense to ask whether these geodesic flows on Riemannian locally symmetric spaces are the only examples of geodesic flows on manifolds of negative curvature with smooth horospheric (Anosov) foliations.

Concerning this question, the first result that yields information about manifolds of a arbitrary dimension was proven by M. Kanai:

Proposition $2[K]$. Let $M$ be a compact $C^{\infty}$ Riemannian manifold of dimension 
$n \geq 2$. Assume that the sectional curvature of $M$ satisfies

$$
-\frac{9}{4}<K \leq-1
$$

and that the Anosov foliations in the unit tangent bundle $V$ of $M$ are $C^{\infty}$. Then the geodesic flow $\varphi_{t}: V \rightarrow V, t \in \mathbb{R}$, is $C^{\infty}$-isomorphic to the geodesic flow $\hat{\varphi}_{t}: \hat{V} \rightarrow \hat{V}$ on the unit tangent bundle $\hat{V}$ of a closed Riemannian manifold $\hat{M}$ of constant negative curvature, i.e., there exists a $C^{\infty}$ diffeomorphism $\Phi: V \rightarrow \hat{V}$ such that $\Phi \circ \varphi_{t}=\hat{\varphi}_{t} \circ \Phi$.

The main purpose of the present work is to improve Kanai's result by relaxing the requirement on the sectional curvature $\mathrm{K}$.

Before we explain the weaker conditions under which Proposition 2 holds, we observe that (1) If $M$ is a Riemannian locally-symmetric space of strictly negative curvature, which does not have constant curvature, its sectional curvature $\mathrm{K}$ must satisfy

$$
\frac{\sup K}{\inf K}=\frac{1}{4}
$$

(2) If the dimension of $M$ is odd ( $M$ being a Riemannian locally-symmetric space of negative curvature) then it is locally isometric to $\mathbb{R H}^{n}$.

Therefore the optimal assumptions on $\mathrm{K}$ in order to have the same conclusion as in Proposition 2 are expected to be: (1) either the sectional curvature $\mathrm{K}$ must satisfy the pinching condition

$$
-4<K \leq-1
$$

(we can always achieve sup $K=-1$ by a homothetic change of metric) or (2) If the dimension of $M$ is odd, no restriction on $K$ should be necessary.

It turns out that these conditions are enough:

Theorem. Let $M$ be a closed $C^{\infty}$ Riemannian manifold of negative sectional 
curvature and dimension greater than two. Assume the stable or unstable foliation of the geodesic flow $\varphi_{t}: V \rightarrow V$ on the unit tangent bundle $V$ of $M$ is $C^{\infty}$. Assume moreover that either (a) $-4<K \leq-1$ or else (b) the dimension of $M$ is odd. Then the geodesic flow of $M$ is $C^{\infty}$-isomorphic to the geodesic flow on a closed Riemannian manifold of constant negative curvature.

The proof of the Theorem under condition (a) was given in [FK1] by A. Katok and myself (it will follow from our Proposition 13). Some of the techniques used in case (b) generalize ideas already employed in our two papers [FK1] and [FK2]. This case constitutes the core of the present paper.

The organization of this work is as follows. In section II, we collect a number of basic facts concerning geodesic flows and Anosov flows and define the notation we will be using throughout this work.

In section III we define the fundamental technical object of our analysis - an invariant affine connection on $\mathrm{V}$, for a contact Anosov flow $\varphi_{t}: \mathrm{V} \rightarrow \mathrm{V}$. This connection, introduced (in a slightly different way than in this paper) by $M$. Kanai in [K], is a smooth object if the Anosov foliations are themselves smooth.

Section IV is dedicated to a brief summary of Kanai's work. The core of his work is the proof that if the invariant connection $\nabla$ (defined in section III) is locally symmetric (i.e., $\nabla \mathrm{R} \equiv 0, \mathrm{R}$ being the curvature tensor associated to $\nabla$ ) then the conclusion of Proposition 2 holds. Therefore our task should be to establish $\nabla R \equiv 0$ under the weaker assumptions of the theorem.

The purpose of section $\mathrm{V}$ is to relate the existence of nontrivial invariant smooth tensor fields on $\mathrm{V}$, like $\nabla \mathrm{R}$ (assuming it does not vanish), to properties of the 
Lyapunov exponents and the Oseledec decomposition of TV (these notions are defined at the beginning of section $\mathrm{V}$ ). The fundamental result obtained there is that, if the Anosov foliations of a contact Anosov flow are $C^{\infty}$ and there exists a $C^{\infty}$ tensor field $\tau$, which is invariant and such that $\nabla \tau \neq 0$, then there exists a smooth, flow invariant distribution of $k$-planes contained in $\mathrm{E}^{+}$or $\mathrm{E}^{-}$, where $1 \leq \mathrm{k} \leq \mathrm{dim} \mathrm{E}^{+}-1$. Moreover, it is shown that there exists no invariant $\mathrm{C}^{\infty}$ tensor field $\tau$ on $\mathrm{V}$ such that $\nabla \tau \neq 0$ if we assume that at almost every point the maximal Lyapunov exponent is strictly less than twice the minimum positive Lyapunov exponent.

Finally, in section VI we conclude the proof of the Theorem. Under the hypothesis $M=$ odd, which is the case not considered in our previous works [FK1] and [FK2], this is accomplished by showing how the distributions mentioned above (obtained from the assumption $\nabla R \neq 0$ ) can be used to construct a continuous nontrivial subbundle of an $\mathrm{n}$-1-dimensional sphere. Since this is impossible if $\mathrm{n}$ is odd, we obtain a contradiction, which yields the proof in case (b).

More generally, as pointed out before, if no restriction on the sectional curvature $K$ is assumed, then one expects to have:

Conjecture. Let $M$ be a closed $C^{\infty}$ Riemannian manifold of negative sectional curvature and dimension greater than two. Assume the Anosov foliations of the geodesic flow $\varphi_{t}: V \rightarrow V$ on the unit tangent bundle $V$ of $M$ are $C^{\infty}$. Then the geodesic flow of $M$ is $C^{\infty}$-isomorphic to the geodesic flow on a closed locally-symmetric Riemannian manifold of negative curvature.

We collect in an appendix at the end a few scattered results, which provide some evidence to this conjecture in the particular case of manifolds of dimension $\equiv 2(\bmod 4)$. 


\section{Definitions, Notations, and Basic Facts}

In this section we mention a number of basic facts about geodesic flows and Anosov flows and prove a simple technical fact (Proposition 6) concerning the extension of invariant distributions of $k$-planes, needed in the proof of the Theorem.

For the reader's convenience, we cast below the notation we are going to use throughout the work.

\section{Notation}

M - a closed (compact, boundary-less) smooth (that is, $\mathrm{C}^{\infty}$ ) manifold

$\sigma$ - a smooth Riemannian metric on $\mathrm{M}$

$\pi^{\prime}: \mathrm{TM} \rightarrow \mathrm{M}-$ the tangent bundle of $\mathrm{M}$

$\pi: \mathrm{TTM} \rightarrow \mathrm{TM}$ - the tangent bundle of TM

$\mathrm{V}=\left\{\mathrm{v} \in \mathrm{TM}: \sigma_{x}(\mathrm{v}, \mathrm{v})=1, \mathrm{x}=\pi^{\prime}(\mathrm{v})\right\}$ - the unit tangent bundle of $\mathrm{M}, \sigma$. At times, $\mathrm{V}$ will denote a more general smooth manifold carrying an Anosov flow.

$\widetilde{M}, \widetilde{V}$ - the universal coverings of $M, V$

$\Gamma$ - the fundamental group of $M$, represented as a discrete subgroup of the isometry group of $\tilde{M} . \Gamma$ also acts on $\widetilde{V}$ and we have $M=\tilde{M} / \Gamma, V=\widetilde{V} / \Gamma$.

$\mathrm{J}: \mathrm{V} \rightarrow \mathrm{V}$ - the flip map. $\mathrm{J}$ is the diffeomorphism of $\mathrm{V}$, which sends $\mathrm{v} \in \mathrm{V} \rightarrow-\mathrm{v}$.

$\theta$ - the contact form of TM or V

$\Omega$ - the symplectic form of TM or E

$\varphi_{t}: \mathrm{TM} \rightarrow \mathrm{TM}\left(\varphi_{t}: \mathrm{V} \rightarrow \mathrm{V}\right), \mathrm{t} \in \mathbb{R}$ - the geodesic flow of $(\mathrm{M}, \sigma)$. It will also denote, at times, a more general Anosov flow on a manifold V.

$\dot{\varphi}$ - the vector field generating the flow, i.e., $\dot{\varphi}(\mathrm{v})=\left.\frac{\mathrm{d}}{\mathrm{dt}}\right|_{t=0} \varphi_{t}(\mathrm{v})$

$\mathrm{TV}=\mathrm{E}^{+} \oplus \mathrm{E}^{-} \oplus \mathrm{E}^{0}-$ the Anosov splitting of $\mathrm{V}$

$\lambda=|\theta \wedge \mathrm{d} \theta \wedge \cdots \wedge \mathrm{d} \theta|=\left|\theta \wedge \Omega^{n-1}\right|$ - the Liouville measure on V. $\lambda$ is a geodesic flow- 
invariant, finite, smooth measure on $\mathrm{V}$

$\mathrm{E}^{+}\left(\mathrm{W}^{+}\right)$- the strong unstable distribution (foliation)

$\mathrm{E}^{-}\left(\mathrm{W}^{-}\right)$- the strong stable distribution (foliation)

$\mathrm{E}^{+0}=\mathrm{E}^{+} \oplus \mathrm{E}^{0}\left(\mathrm{~W}^{+0}\right)$ - the weak unstable distribution (foliation)

$\mathrm{E}^{-0}=\mathrm{E}^{-} \oplus \mathrm{E}^{0}\left(\mathrm{~W}^{-0}\right)$ - the weak stable distribution (foliation)

$\mathrm{E}=\mathrm{E}^{-} \oplus \mathrm{E}^{+}$

$\mathrm{P}=\widetilde{\mathrm{V}} / \varphi-$ the space of orbits of $\varphi_{t}: \widetilde{\mathrm{V}} \rightarrow \widetilde{\mathrm{V}}$

$\mathrm{p}: \widetilde{\mathrm{V}} \rightarrow \mathrm{P}$ - the natural projection

$\mathrm{TP}=\mathrm{F}^{+} \oplus \mathrm{F}^{-}-$the hyperbolic splitting of TP

$\epsilon$ - the sign + or -

$\mathrm{F}^{\epsilon}=\mathrm{Dp} \mathrm{E}^{\epsilon}$

$\mathscr{F}^{\epsilon}-$ the foliation associated to $\mathrm{F}^{\epsilon}$

B - the ideal boundary of the manifold $\widetilde{M}$ (of negative curvature)

$36_{u v}^{\gamma}$ - the holonomy map ( or canonical map) along a curve $\gamma$ contained in some (weak)

stable or unstable leaf joining the points $u$ and $v$

$\mathrm{H}_{u v}^{\gamma}(\mathrm{w})$ - the differential of $\mathfrak{K}_{u v}^{\gamma}(\mathrm{w})$ at $\mathrm{w}$ (if $\mathrm{w}$ is omitted, the differential is understood to be taken at $v$ itself)

$\pi^{\epsilon}: \mathrm{TV} \rightarrow \mathrm{E}^{\epsilon}, \epsilon=+$ or -- the linear projections

$\nabla$ - the Kanai connection on $\mathrm{V}(\tilde{\mathrm{V}})$

$\nabla^{p}$ - the Kanai connection on $\mathrm{P}$

$\mathrm{x}, \mathrm{y}, \mathrm{z}$, etc. - points of $\mathrm{M}$

$\mathrm{u}, \mathrm{v}, \mathrm{w}$, etc. -points of $\mathrm{V}$

$\xi, \eta, \zeta$, etc. - tangent vectors (vector fields) of $\mathrm{V}$

$\xi^{*}$ - the horizontal lift to $\widetilde{V}$ of the vector field $\xi$ on $P$

$\mathrm{C}_{i n v}^{l}\left(\stackrel{r}{\otimes} \mathrm{E}^{*}\right)$ - the set of flow-invariant $\mathrm{C}^{l}$ tensor fields $\tau$ on $\mathrm{V}$ of type $(0, \mathrm{r})$ such that 
$\tau\left(\xi_{1}, \cdots, \xi_{r}\right)=0$ if $\xi_{i} \in \mathrm{E}^{0}$ for some $\mathrm{i}$

$W_{q}^{\epsilon}(v)$ - the connected component of $W^{\epsilon}(v) \cap u$ containing $v$

$W^{\epsilon}(v)$ - the leaf of $W^{\epsilon}$ containing $v$

$\mathrm{L}^{\perp}$ - the skew-orthogonal complement of $\mathrm{L}$ with respect to the symplectic form $\Omega$

$£_{\xi}$ - the Lie derivative along the vector field $\xi$

$\mathcal{Q}, \mathcal{\Upsilon}, \mathcal{W}$, etc. - open sets in $\mathrm{V}$ or in $\mathrm{P}$

Remark. The following notation will also be used frequently: If $\mathrm{L}$ and $\mathrm{N}$ are smooth distributions of linear spaces in $T V$, then $\nabla_{L} N$, [L, N], etc., will stand for the set of all vector fields $\nabla_{\xi} \eta,[\xi, \eta]$, etc., such that $\xi \in L, \eta \in N$.

Geodesic Flows. (see, e.g., [Kl]) For a given closed $\mathrm{C}^{\infty}$ Riemannian manifold $\mathrm{M}$ with a complete $\mathrm{C}^{\infty}$ metric $\sigma$, define the contact form $\theta$ as the smooth 1 -form on TM, $\theta \in \mathrm{C}^{\infty}\left(\mathrm{T}^{*} \mathrm{TM}\right)$, such that for $\mathrm{v} \in \mathrm{TM}, \pi^{\prime}(\mathrm{v})=\mathrm{x}$, and for each $\xi \in \mathrm{T}_{v} \mathrm{TM}$,

$$
\theta_{v}(\xi)=\sigma_{x}\left(\mathrm{v}, \mathrm{D} \pi^{\prime}{ }_{v} \xi\right)
$$

where $\mathrm{D} \pi^{\prime}{ }_{v}: \mathrm{T}_{v} \mathrm{TM} \rightarrow \mathrm{T}_{x} \mathrm{M}$ denotes the differential of the projection map $\pi^{\prime}$ at $\mathrm{v}$. The same symbol $\theta$ will refer to the restriction of the contact form to $\mathrm{V}$ - the unit tangent bundle of $\mathbf{M}$.

Let $\Omega$ be the smooth 2 -form on TM defined by

$$
\Omega=\mathrm{d} \theta \in \mathrm{C}^{\infty}\left(\Lambda^{2} \mathrm{~T}^{*} \mathrm{TM}\right) \text {. }
$$

$\Omega$ defines a symplectic form on $\mathrm{TM}$, that is, it is a closed, nondegenerate 2-form on TM. In particular $\Omega^{n}=\Omega \wedge \cdots \wedge \Omega$ is nonzero everywhere, for $n=\operatorname{dim} M$.

The geodesic flow $\varphi_{t}: \mathrm{TM} \rightarrow \mathrm{TM}\left(\varphi_{t}: \mathrm{V} \rightarrow \mathrm{V}\right), \mathrm{t} \in \mathbb{R}$ - or simply $\varphi$ - is the flow on TM generated by the vector field $\dot{\varphi}$ given as follows. Consider the smooth function $\mathrm{E}: \mathrm{TM} \rightarrow \mathbb{R}$ defined by

$$
\mathrm{E}(\mathrm{v})=\frac{1}{2} \sigma_{x}(\mathrm{v}, \mathrm{v}),
$$


for $\mathrm{x}=\pi^{\prime}(\mathrm{v})$ (so that $\mathrm{V}=\{\mathrm{v} \in \mathrm{TM}: \mathrm{E}(\mathrm{v})=1 / 2\}$ ). Denote by $\dot{\varphi}$ the Hamiltonian vector field associated with $\mathrm{E}$; that is to say that $\dot{\varphi}$ is defined by the equation $\Omega(\dot{\varphi}, \cdot)=\mathrm{dE}$. Let $\varphi_{t}: \mathrm{TM} \rightarrow \mathrm{TM}, \mathrm{t} \in \mathbb{R}$, be the flow on $\mathrm{TM}$, which integrates $\dot{\varphi}$. Observe that

$$
\frac{\mathrm{d}}{\mathrm{dt}} \mathrm{E}\left(\varphi_{t}(\mathrm{v})\right)=\mathrm{DE}_{\varphi_{t}(v)} \dot{\varphi}\left(\varphi_{t}(\mathrm{v})\right)=\Omega_{\varphi_{t}(v)}(\dot{\varphi}, \dot{\varphi})=0,
$$

hence $\mathrm{E}\left(\varphi_{t}(\mathrm{v})\right)$ is constant as a function of $\mathrm{t}$ and $\varphi_{t}$ can therefore be restricted to the unit tangent bundle $\mathrm{V}$ of $\mathrm{M}$. It can be shown that the flow lines $\varphi_{t}(\mathrm{v})$ of the geodesic flow associated to a complete Riemannian metric are defined for all $t \in \mathbb{R}$ and they project under $\pi^{\prime}$ onto geodesics in $M$ with initial velocity $\mathrm{v}$.

The forms $\theta$ and $\Omega$ are geodesic flow-invariant, i.e.,

$$
\varphi_{t}^{*} \theta=\theta, \varphi_{t}^{*} \Omega=\Omega \text {, }
$$

([Kl] Proposition 3.1.10) where $\varphi_{t}{ }^{*}$ denotes the pull-back of forms. $\theta$ and $\Omega$ will be viewed as forms on $\mathrm{V}$ and we will only consider the geodesic flow $\varphi_{t}: \mathrm{V} \rightarrow \mathrm{V}$ on the unit tangent bundle of $\mathrm{M}$ rather than on TM.

The tangent bundle TV of $\mathrm{V}$ possesses a smooth flow-invariant direct sum decomposition $\mathrm{TV}=\mathrm{E} \oplus \mathrm{E}^{0}$ into smooth subbundles $\mathrm{E}$ and $\mathrm{E}^{0}$, where $\mathrm{E}^{0}$ is the onedimensional line field spanned by $\dot{\varphi}$. Here, $\oplus$ denotes the Whitney sum of subbundles and by flow invariance we mean, for example, $\mathrm{D} \varphi_{t} \mathrm{E}(\mathrm{v})=\mathrm{E}\left(\varphi_{t}(\mathrm{v})\right)$ for all $\mathrm{t} . \mathrm{E}(\mathrm{v})$ is the $\Omega$-skew complement of $\mathrm{E}^{0}(\mathrm{v})$ in $\mathrm{T}_{v} \mathrm{TM}$, i.e.,

$$
\mathrm{E}(\mathrm{v})=\mathrm{E}^{0}(\mathrm{v})^{\perp}=\left\{\xi \in \mathrm{T}_{v} \mathrm{TM}: \Omega(\xi, \dot{\varphi}(\mathrm{v}))=0\right\} .
$$

$\Omega$ restricted to $\mathrm{E}(\mathrm{v})$ is nondegenerate for all $\mathrm{v}$ and we also have

$$
\mathrm{E}(\mathrm{v})=\left\{\xi \in \mathrm{T}_{v} \mathrm{~V}: \theta(\xi)=0\right\}
$$

In particular

$$
\lambda=\left|\theta \wedge \Omega^{n-1}\right|
$$

defines a flow-invariant volume element on $\mathrm{V}$, sometimes called the Liouville measure. 
The Flip Map. It is the diffeomorphism of $\mathrm{V}$ defined by $\mathrm{J} v=-\mathrm{v}$. The basic

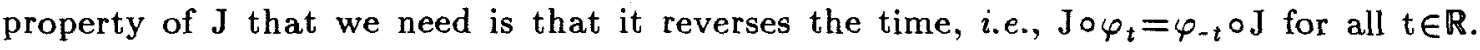
Taking this as the defining property of $\mathrm{J}$, we immediately obtain:

(i) $(\mathrm{DJ}) v \dot{\varphi}(\mathrm{v})=-\dot{\varphi}(\mathrm{J}(\mathrm{v}))$

(ii) (DJ) $v \mathrm{E}^{\epsilon}(\mathrm{v})=\mathrm{E}^{-\epsilon}(\mathrm{J}(\mathrm{v})),(\mathrm{DJ}){ }_{v} \mathrm{~W}^{\epsilon}(\mathrm{v})=\mathrm{W}^{-\epsilon}(\mathrm{J}(\mathrm{v}))$.

(iii) $\mathrm{J}^{*} \theta=-\theta, \mathrm{J}^{*} \Omega=-\Omega$.

From now on, $\sigma$ will denote a Riemannian metric of strictly negative curvature on M. The fundamental fact regarding the dynamics of geodesic flows on negatively curved manifolds is expressed in Proposition 1, which characterizes these flows as being Anosov flows. Since most of our work actually belongs to the more general setting of contact Anosov flows, we would like to devote the next paragraph to this class of systems.

Contact Anosov Flows. Let $\varphi_{t}: \mathrm{V} \rightarrow \mathrm{V}$ be an Anosov flow on a closed smooth manifold $\mathrm{V}$ of dimension $2 \mathrm{n}-1$. In the following discussion assume that the Anosov foliations are of class $C^{r}$, for $r \geq 1$.

We say that the flow $\varphi$ is a contact flow if $V$ possesses a smooth 1 -form $\theta \in \mathrm{C}^{\infty}\left(\mathrm{T}^{*} \mathrm{~V}\right)$ such that

(a) $\theta$ is $\varphi$-invariant,

(b) $\mathrm{d} \theta$ is nondegenerate on $\mathrm{E}=\mathrm{E}^{+} \oplus \mathrm{E}^{-}$and

(c) $\theta(\dot{\varphi}(\mathrm{v}))=1$.

We have the following easy observations:

(i) $\theta(E)=0$. In fact, if $\theta$ is any continuous flow invariant 1 -form on $V, \xi \in E^{\epsilon}(v)$ for $\epsilon \in\{+,-\}$ and $v \in V$ then for all $t \geq 0$ 


$$
\left|\theta_{v}(\xi)\right|=\left|\left(\varphi_{-\epsilon t}^{*} \theta\right)_{v}(\xi)\right|=\left|\theta_{\varphi_{-\epsilon t}(v)}\left(\mathrm{D} \varphi_{-\epsilon t} \xi\right)\right| \leq\|\theta\| \cdot\left\|\mathrm{D} \varphi_{-\epsilon t} \xi\right\| \leq \text { const. }\|\xi\| \cdot e^{-a t}
$$

so that $\theta_{v}(\xi)=0$. It follows that there exists at most one differential 1-form on $\mathrm{V}$ satisfying (a), (b), and (c) above.

(ii) $\mathrm{d} \theta\left(\mathrm{E}^{\epsilon}, \mathrm{E}^{\epsilon}\right)=0$ and $\mathrm{d} \theta\left(\mathrm{E}^{0}, \mathrm{E}^{\epsilon}\right)=0$, for $\epsilon=+$ and - . This is shown exactly as in (i). In particular, $\mathrm{E}^{+}$and $\mathrm{E}^{-}$are Lagrangian distributions with respect to the symplectic form $\Omega=\left.\mathrm{d} \theta\right|_{\mathrm{E}}$. Since $\Omega$ is nondegenerate on $\mathrm{E}$ and $\Omega\left(\mathrm{E}^{\epsilon 0}, \mathrm{E}^{\epsilon 0}\right)=0$, one obtains a linear isomorphism between $\mathrm{E}^{\epsilon}$ and $\left(\mathrm{E}^{-\epsilon}\right)^{*}$, given by

$$
\xi \in \mathrm{E}^{\epsilon}(\mathrm{v}) \rightarrow \Omega(\xi, \cdot) \in\left(\mathrm{E}^{-\epsilon}(\mathrm{v})\right)^{*}
$$

$\mathrm{E}^{+}$and $\mathrm{E}^{-}$therefore have the same dimension.

(iii) $\theta \wedge \mathrm{d} \theta \wedge \cdots \wedge \mathrm{d} \theta=\theta \wedge(\mathrm{d} \theta)^{n-1} \neq 0(\operatorname{dim} \mathrm{V}=2 \mathrm{n}-1)$. This is immediate from the assumption that $\mathrm{d} \theta$ is nondegenerate on $\mathrm{E}$ and from (i) and (ii). It follows that $\lambda=\left|\theta \wedge(\mathrm{d} \theta)^{n-1}\right|$ is a flow-invariant volume element on $\mathrm{V}$.

Other properties of Anosov flows are collected in the following proposition.

Proposition 3 (Anosov [A]) If $\varphi_{t}: V \rightarrow V$ is a $C^{\infty}$ Anosov flow on a compact manifold $V$, which preserves a smooth probability measure $\lambda$, then

(i) $\lambda$ is an ergodic measure

(ii) The set of periodic points is dense in $V$

(iii) For all $v \in V, W^{\epsilon 0}(v)$ (the leaf of $W^{\epsilon 0}$ through $v$ ) is dense in $V$ for $\epsilon=+$ and - . For contact flows all the leaves $W^{\epsilon}$ are also dense in $V$.

Next, we focus our attention on the Anosov foliations $W^{\epsilon}$ and $W^{\epsilon 0}$.

Holonomy. The foliations $\mathrm{W}^{+}$and $\mathrm{W}^{-}$are assumed, here, to be $\mathrm{C}^{r}$ for $\mathrm{r} \geq 1$. Observe that, in the case of geodesic flows, these foliations must have the same regularity since they are permuted by the diffeomorphism $\mathrm{J}$. 
$\mathrm{W}^{\epsilon}$ and $\mathrm{W}^{-\epsilon}$ are transverse to each other and have complementary dimensions ( $\mathrm{n}-1$ and $\mathrm{n}$, respectively).

By a foliation chart $(f, \mathcal{U})$ we mean a $C^{r}$ diffeomorphism $f$ of an open subset $\mathcal{U}$ of $V$ onto the cube $I^{n-1} \times I^{n}$, where $I$ is the interval $\{t \in \mathbb{R}:-1<t<1\}$, and $f$ : $\mathrm{x} \rightarrow\left(\mathrm{f}_{1}(\mathrm{x}), \mathrm{f}_{2}(\mathrm{x})\right) \in \mathrm{I}^{n-1} \times \mathrm{I}^{n}$ is such that the connected components of $\mathrm{W}^{\epsilon} \cap \mathcal{U}$ are given by $f_{2}=$ constant and the connected components of $W^{-\epsilon 0} \cap \mathcal{Q}$ are given by $f_{1}=$ constant.

Let $\mathcal{U}$ be the domain of a foliation chart (also called, here, a foliated domain) for $\mathrm{W}^{\epsilon}$ and $\mathrm{W}^{-\epsilon 0}$ and consider two points $\mathrm{v}$ and $\mathrm{w}$ in $\mathcal{U}$ belonging to the same connected component of $W^{\epsilon} \cap \mathcal{~}$. We write $w \in W_{\mathcal{U}}^{\epsilon}(v)$.

Define the holonomy map (or canonical map) along the foliation $\mathrm{W}^{-\epsilon 0}$ to be the $\mathrm{C}^{r}$-diffeomorphism (see the figure)

$$
J_{w v}^{\mathcal{u}}: \mathrm{u} \in \mathrm{W}_{\mathcal{U}}^{\epsilon}(\mathrm{v}) \mapsto \mathrm{u}^{\prime} \in \mathrm{W}_{\mathcal{u}}^{-\epsilon 0}(\mathrm{u}) \cap \mathrm{W}_{\mathcal{U}}^{\epsilon}(\mathrm{w})
$$

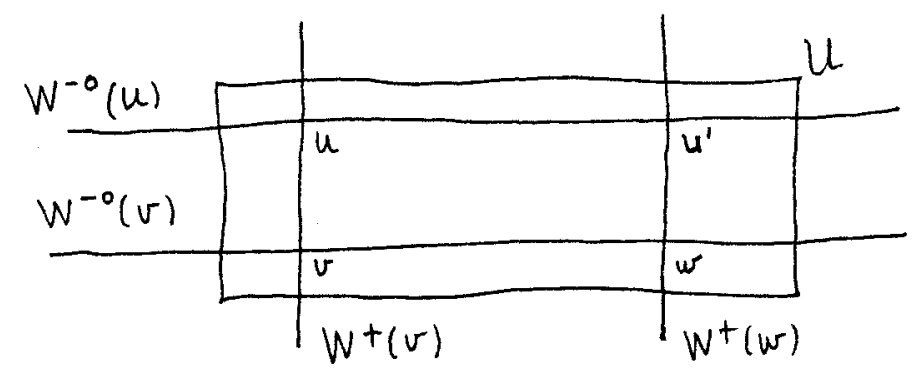

More generally, if $\mathrm{w} \in \mathrm{W}^{-\epsilon 0}(\mathrm{v})$ and $\gamma$ is a smooth curve joining $\mathrm{w}$ and $\mathrm{v}$ such that $\gamma(\mathrm{t}) \in \mathrm{W}^{-\epsilon 0}(\mathrm{v})$ for all $\mathrm{t}$, we can define $J_{w v}^{\gamma}$ by covering the image of $\gamma$ with sets $\mathcal{Q}_{i}, \mathrm{i}=1, \cdots, \mathrm{k}$, where one can define $\jmath_{6}{ }^{\mathcal{U}_{i}}$, and taking the composition 


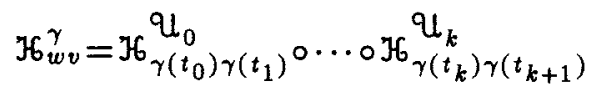

so that $\gamma\left(t_{i}\right), \gamma\left(t_{i+1}\right) \in \mathcal{U}_{i}\left(J^{\gamma}\right.$ only depends on the homotopy class of $\left.\gamma \in \mathrm{W}^{-\epsilon 0}(\mathrm{v})\right)$.

The following two properties are immediate consequences of the definitions.

(i) $3 G_{w v}^{\gamma} \circ 3 G_{v u}^{\gamma}=3 G_{w u}^{\gamma}$, for $\mathrm{w}, \mathrm{v}$ and $\mathrm{u}$ on the image of $\gamma$ (the composition being welldefined, if we take the domain of $\mathcal{F}_{v u}^{\gamma}$ appropriately small).

(ii) $\varphi_{t} \circ J_{f_{v}}^{\gamma} \circ \varphi_{-t}=J_{\varphi_{t}}^{\varphi_{t} \circ \gamma}(w) \varphi_{t}(v)$, for all $t \in \mathbb{R}$. This property follows from the flowinvariance of the Anosov foliations.

It is a well-known fact (and trivial, in our case, since the holonomy maps are differentiable) that $J_{w v}^{\gamma}$ is absolutely continuous with respect to the Lebesgue measure on leaves.

We can also consider the linear isomorphism

$$
\mathrm{H}_{w v}^{\gamma}(\mathrm{u})=\left(\mathrm{D} \mathcal{K}_{w v}^{\gamma}\right)_{u}: \mathrm{E}^{\epsilon}(\mathrm{u}) \rightarrow \mathrm{E}^{\epsilon}\left(\mathcal{H}_{w v}^{\gamma}(\mathrm{u})\right) .
$$

(The above constructions are clearly also defined on the universal covering $\widetilde{V}$ of $V$. .)

We say that an object defined on the leaves of $\mathrm{W}^{\epsilon}$ is invariant under the holomy transport along the leaves of $\mathrm{W}^{-\epsilon 0}$ if it is invariant under the map $J_{6}^{\gamma}$ just defined.

The Space of Leaves $\widetilde{V} / W^{\epsilon 0}$. (See also $[K]$ and references therein.) In $\tilde{V}$, define the equivalence relation that identifies points on a same leaf of $W^{\epsilon 0}$. The quotient, equipped with the quotient topology, can naturally be called the space of leaves of $\mathrm{W}^{\epsilon 0}$ (in $\widetilde{V}$ ) and will be denoted by $\widetilde{V} / W^{\epsilon 0}$.

$\widetilde{V} / W^{+0}$ and $\widetilde{V} / W^{-0}$ are not necessarily well-behaved (say, Hausdorff) spaces for a general Anosov flow. However, for the geodesic flow on the unit tangent bundle of a complete Riemannian manifold $M$ of negative curvature the following is true: $\widetilde{V} / W^{+0}$ 
and $\tilde{V} / W^{+0}$ are homeomorphic to an $(n-1)$-dimensional sphere, which can be naturally identified with the ideal boundary $B$ of $\widetilde{M}(\operatorname{dim} M=n)$. If the foliations are $C^{r}, r \geq 1$, then $\widetilde{V} / W^{+0}$ and $\widetilde{V} / W^{-0}$ are $C^{r}$-diffeomorphic to an $(n-1)$-dimensional sphere (observe that if we use local leaves of $\mathrm{W}^{-}$to define coordinate systems for $\tilde{\mathrm{V}} / \mathrm{W}^{+0}$, then the change of coordinates is given by the holonomy map).

Note that if $v \in \widetilde{V} \mapsto F(v) \subset E^{\epsilon}(v)$ is a continuous distribution of linear subspaces of $\mathrm{E}^{\epsilon}$, which is invariant under the holonomy transport along the leaves of $\mathrm{W}^{-\epsilon 0}$, then $\mathrm{F}$ descends to a continuous distribution on $\tilde{\mathrm{V}} / \mathrm{W}^{-\epsilon 0}$.

The Space of Orbits $P[K]$. Denote by $P$ the quotient space $\widetilde{V} / \varphi$, where two points on $\widetilde{V}$ are identified if and only if they lie on a same orbit of $\varphi$.

If $\mathrm{V}$ is the unit tangent bundle of a complete Riemannian manifold of negative curvature, its space $P$ has a unique differential structure that makes the natural projection $p: \widetilde{V} \rightarrow P$ smooth and it can be identified (is homeomorphic) with the space

$$
\left\{\left(\mathrm{b}^{+}, \mathrm{b}^{-}\right): \mathrm{b}^{+}, \mathrm{b}^{-} \in \mathrm{B} \text { and } \mathrm{b}^{+} \neq \mathrm{b}^{-}\right\} \sim \mathrm{S}^{n-1} \times \mathrm{S}^{n-1} \text {-diagonal }
$$

where $B$ is the ideal boundary of $\tilde{M}$. Observe that $P$ is simply connected if $n \geq 3$.

We also have the following facts:

(i) The exterior derivative $\mathrm{d} \theta$ of the contact form $\theta$ on $\widetilde{\mathrm{V}}$ can be pushed forward to define a symplectic form $\Omega$ on $P$ (this is because $d \theta$ is flow invariant and $\mathrm{d} \theta(\dot{\varphi}, \cdot) \equiv 0)$

(ii) The distributions $\mathrm{F}^{+}$and $\mathrm{F}^{-}$are integrable. Denote by $\mathcal{F}^{+}$and $\mathcal{F}^{-}$the corresponding foliations. They constitute a pair of transverse Lagrangian foliations with respect to $\Omega$.

The Holonomy Span of a Distribution. In this paragraph, let $\varphi_{t}: \mathrm{V} \rightarrow \mathrm{V}$ denote 
any $\mathrm{C}^{\infty}$ Anosov flow on a compact smooth manifold $\mathrm{V}$ possessing a smooth, flowinvariant volume form $\lambda$.

Given a distribution of linear spaces

$$
\mathrm{v} \in \mathrm{U} \subset \mathrm{V} \mapsto \mathrm{K}(\mathrm{v}) \subset \mathrm{E}^{\epsilon}(\mathrm{v})
$$

defined on an open subset $น \neq \emptyset$, we can define the holonomy span of $\mathrm{K}$, denoted by hspan $\mathrm{K}$, as

$$
\begin{gathered}
\mathrm{v} \in \mathrm{V} \mapsto(\mathrm{h} \operatorname{span} \mathrm{K})(\mathrm{v})=\operatorname{span}\left\{\mathrm{H}_{v u}^{\gamma}(\mathrm{u}) \mathrm{K}(\mathrm{u}): \mathrm{u} \in \mathcal{U} \cap \mathrm{W}^{-\epsilon 0}(\mathrm{v}) \text { and } \gamma\right. \text { is } \\
\text { a smooth curve in } \left.\mathrm{W}^{-\epsilon 0}(\mathrm{v}) \text { joining } \mathrm{w} \text { and } \mathrm{v}\right\} .
\end{gathered}
$$

In words: For each $v \in V$ and for every $u \in \mathcal{U}$ belonging to the weak $\epsilon$-leaf of $v$, consider the holonomy transport of $K(u)$ from $u$ to $v$. The linear span of all the subspaces of $E^{\epsilon}(v)$ obtained in this way is by definition $(h \operatorname{span} K)(v)$.

Proposition 6. Let $\varphi_{t}: V \rightarrow V(t \in \mathbb{R})$ denote any $C^{\infty}$ Anosov flow on a closed manifold $V$, preserving a smooth probability measure $\lambda$. Let $E^{\epsilon}$ and $E^{\epsilon 0}$ for $\epsilon=+,-$ denote the tangent bundles of $W^{\epsilon}$ and $W^{\epsilon 0}$. Assume the Anosov foliations are $C^{r}$, $r \geq 1$, and let $v \in \mathcal{U} \mapsto K(v) \subset E^{\epsilon}$, for some $\epsilon, a C^{r-1}$ distribution of linear subspaces of $E^{\epsilon}$ defined on an open subset $น \neq \emptyset$ of $V$. Let $v \in V \mapsto F(v)$ be a measurable distribution of linear subspaces of $E^{\epsilon}$ such that $F$ is invariant under the holonomy transport along the leaves of $W^{-\epsilon 0}$ and assume that $K(v) \subset F(v)$ for almost every point $v$ in 4 . Then $u \in V \mapsto L(v)=h \operatorname{span} K(u)$ is a $C^{r-1}$ distribution, which is invariant under the holonomy transport along $W^{-\epsilon 0}$ and for almost every $u \in V, L(u) \subset F(u)$. Moreover, if $K$ is flowinvariant, so is $L$.

Proof. Let $v \in V$ be a point where $L=h \operatorname{span} K$ has the maximal dimension. There exists a neighborhood $\checkmark$ of $\mathrm{v}$ where the dimension of $\mathrm{L}$ does not drop.

If $\mathrm{u}$ is any other point in $\mathrm{V}$, we have by Proposition 3 


$$
W^{-\epsilon 0}(v) \cap q \neq \emptyset
$$

so that the dimension of $\mathrm{L}$ is actually constant everywhere, hence $\mathrm{L}$ is continuous everywhere.

Let $\mathrm{v}$ be any point in $\mathrm{V}$. It is also clear (since $E^{\epsilon}(v)$ is finite dimensional) that the holonomy span of $K$ at $v$ can be defined by means of only finitely many points in $\mathrm{W}^{-\epsilon 0}(\mathrm{v})$, that is, one can find points $\mathrm{u}_{0}, \cdots, \mathrm{u}_{k} \in \mathrm{W}^{-\epsilon 0}(\mathrm{v}) \cap \mathrm{u}$ such that $\mathrm{u}_{0}=\mathrm{v}, \mathrm{u}_{i}=\gamma\left(\mathrm{t}_{i}\right)$ for a smooth curve $\gamma \subset W^{-\epsilon 0}(v)$ and

$$
\mathrm{L}(\mathrm{v})=\mathrm{h} \operatorname{span}\left\{\mathrm{H}_{v u_{i}}^{\gamma} \mathrm{K}\left(\mathrm{u}_{i}\right): \mathrm{i}=0, \cdots, \mathrm{k}\right\}
$$

By the continuity of $K$ and the smoothness of the Anosov foliation, there will be an open neighborhood $\mathfrak{u}_{0}$ of $\mathrm{v}$ and $\mathfrak{u}_{i} \subset \mathcal{u}$ of $u_{i}$, for $\mathrm{i}=\mathrm{i} \cdots, \mathrm{k}$, which are sufficiently small so that for any $\mathrm{v}^{\prime} \in \mathcal{U}_{0}$ and any curve $\gamma^{\prime} \subset W^{-\epsilon 0}\left(\mathrm{v}^{\prime}\right)$ close enough to $\gamma$ and any points $u_{i}{ }^{\prime} \in \mathcal{U}_{i}$ on the image of $\gamma$, we have

$$
\mathrm{L}\left(\mathrm{v}^{\prime}\right)=\mathrm{h} \operatorname{span}\left\{\mathrm{H}_{v^{\prime} u_{i}}^{\gamma^{\prime}} \mathrm{K}\left(\mathrm{u}_{i}{ }^{\prime}\right): \mathrm{i}=0, \cdots, \mathrm{k}\right\} \text {. }
$$

Therefore $\mathrm{L}$ is smooth if $\mathrm{K}$ and the holonomy maps are smooth.

Since (a) $K(u) \subset F(u)$ for almost all $u \in \mathbb{Q}$, (b) $F$ is invariant under the holonomy transport along $\mathrm{W}^{-\epsilon 0}$, and (c) the holonomy maps are absolutely continuous, we obtain: For almost all $\mathrm{v}^{\prime} \in \mathcal{U}_{0}$ there will be points $u_{i}^{\prime} \in \mathcal{U}_{i} \cap \mathrm{W}^{-\epsilon 0}\left(\mathrm{v}^{\prime}\right)$ and a smooth curve $\gamma^{\prime}$ close to $\gamma$ such that $u_{i}^{\prime}=\gamma^{\prime}\left(t_{i}^{\prime}\right)$ and

$$
\begin{gathered}
\mathrm{H}_{v^{\prime} u_{i}{ }^{\prime}}^{\gamma^{\prime}} \mathrm{L}\left(\mathrm{u}_{i}{ }^{\prime}\right) \subset \mathrm{F}\left(\mathrm{v}^{\prime}\right), \\
\mathrm{L}\left(\mathrm{v}^{\prime}\right)=\mathrm{h} \operatorname{span}\left\{\mathrm{H}_{v^{\prime} u_{i}{ }^{\prime}}^{\gamma^{\prime}} \mathrm{K}\left(\mathrm{u}_{i}{ }^{\prime}\right): \mathrm{i}=0, \cdots, \mathrm{k}\right\} .
\end{gathered}
$$

Hence $L\left(v^{\prime}\right) \subset F\left(v^{\prime}\right)$ for almost all $v^{\prime} \in \mathcal{U}_{0}$. But $\mathcal{U}_{0}$ was a neighborhood of an arbitrary point of $\mathrm{V}$. Therefore $\mathrm{L} \subset \mathrm{F}$ almost everywhere. 


\section{An Invariant Affine Connection on V}

In this section, unless specified otherwise, $\varphi_{t}: \mathrm{V} \rightarrow \mathrm{V}$ will denote a contact Anosov flow on a compact smooth manifold V.

Denote by $\pi^{\epsilon}: \mathrm{TV} \rightarrow \mathrm{E}^{\epsilon}, \epsilon=+,-$, or 0 , the natural projections and assume the distributions $\mathrm{E}^{\epsilon}$ are differentiable of class $\mathrm{C}^{r}, \mathrm{r} \geq 1$.

The Kanai connection is an affine connection $\nabla$ on $\mathrm{V}$ such that

(i) The 2 -form $\Omega=\mathrm{d} \theta$ is parallel: $\nabla \Omega \equiv 0$. That is to say that given arbitrary smooth vector fields $\xi, \eta$, and $\zeta$ on $\mathrm{V}$ we have $\xi \Omega(\eta, \zeta)-\Omega\left(\nabla \nabla_{\xi} \eta, \zeta\right)-\Omega\left(\eta, \nabla_{\xi} \zeta\right)=0$.

(ii) The projections $\pi^{\epsilon}$ are parallel: $\nabla \pi^{\epsilon} \equiv 0, \epsilon=+,-, 0$. This is equivalent to requiring that if $\xi$ is a vector field such that $\xi(v) \in E^{\epsilon}(v)$ for all $v$ and if $\eta$ is an arbitrary smooth vector field on $\mathrm{V}$, we have

$$
\left(\nabla_{\eta} \xi\right)(\mathrm{v}) \in \mathrm{E}^{\epsilon}(\mathrm{v})
$$

for all $v$. We will simply write $\nabla E^{\epsilon} \subset E^{\epsilon}$.

(iii) $\nabla$ is transversely torsion-free, meaning that the torsion tensor $\mathrm{T}$ of $\nabla$ only has nonzero component along $\mathrm{E}^{0}$ (thus $\mathrm{T}(\xi, \eta)=\mathrm{f}(\xi, \eta) \cdot \dot{\varphi}$, where $\mathrm{f}$ is an antisymmetric bilinear function).

(iv) $\dot{\varphi}$ is a parallel vector field: $\nabla \dot{\varphi}=0$.

(v) The covariant derivative along $\dot{\varphi}$ is the Lie derivative along the flow.

Remark. The requirements (iv) and (v) are somewhat arbitrary and it would suffice for what follows to define a covariant derivative for vector fields tangent to $\mathrm{E}=\mathrm{E}^{+} \oplus \mathrm{E}^{-}$(derivated along vector fields of the same kind). Yet we find it convenient to have a covariant derivative defined for arbitrary smooth vector fields.

Proposition 7. (i) There exists a unique affine connection $\nabla$ on $V$ satisfying 
$(i)-(v) . \nabla$ is $\varphi_{t}$-invariant and is of class $C^{r-1}$ if the bundles $E^{++}$are of class $C^{r}$.

(ii) Let $\xi \in E^{\epsilon}(v)$ and $w \in W^{-\epsilon 0}(v)$. Consider a differentiable curve $t \mapsto \gamma(t) \in W^{-0}(v)$ such that $\gamma(0)=v$ and $\gamma(1)=w$. Denote by $P_{w v}^{\gamma} \xi$ the parallel transport of $\xi$ to $T_{w} V$ along $\gamma$. Then

$$
P_{w v}^{\gamma} \xi=H_{w v}^{\gamma} \xi
$$

(that is, "parallel transport=holonomy").

Proof. We first show the existence of a unique covariant derivative of vector fields in $\mathrm{E}$, along vectors in $\mathrm{E}$, satisfying the properties (i)-(iii).

Let us define $\mathrm{c}=\pi^{+}-\pi^{-}$and let $\mathrm{g}=\Omega(\cdot, \mathrm{c} \cdot)$. $\mathrm{g}$ is a bilinear, nondegenerate symmetric form on $\mathrm{E}$ and one can define the corresponding Levi-Civita connection - the unique torsion-free connection $\nabla^{\prime}$ with respect to which $\mathrm{g}$ is a parallel tensor field, i.e., $\nabla^{\prime} \mathrm{g} \equiv 0$. Note that $\nabla^{\prime} \mathrm{c} \equiv 0$ is equivalent to $\nabla^{\prime} \pi^{\epsilon} \equiv 0, \epsilon=+,-$, which in turn is equivalent to $\nabla^{\prime}$ preserving the subbundles $E^{\epsilon}, \epsilon=+,-$. To prove this last property, consider the following well-known formulas (see, e.g., [KN] vol.I, p. 36 and [He] Theorem 9.1, p. 48). Given vector fields $\xi, \eta, \nu$ in $\mathrm{E}$,

(a) $2 \mathrm{~g}\left(\nabla^{\prime}{ }_{\xi} \eta, \nu\right)=\xi \mathrm{g}(\eta, \nu)+\eta \mathrm{g}(\xi, \nu)-\nu \mathrm{g}(\xi, \eta)-\mathrm{g}([\eta, \nu], \xi)-\mathrm{g}([\xi, \nu], \eta)+\mathrm{g}([\xi, \eta], \nu)$

(b) $3 \mathrm{~d} \Omega(\xi, \eta, \nu)=\xi \Omega(\eta, \nu)-\eta \Omega(\xi, \nu)+\nu \Omega(\xi, \eta)-\Omega([\eta, \nu], \xi)+\Omega([\xi, \nu], \eta)-\Omega([\xi, \eta], \nu)$.

From (a), (b), and recalling that $\mathrm{d} \Omega=0$ we obtain: For given vector fields $\xi^{\epsilon}$, $\eta^{\epsilon}, \nu^{\epsilon}$ in $\mathrm{E}^{\epsilon}$, for $\epsilon=+,-$

$$
\begin{aligned}
& \mathrm{g}\left(\nabla_{\xi^{\epsilon}}^{\prime} \eta^{\epsilon}, \nu^{\epsilon}\right)=0, \text { hence } \nabla_{\xi^{\epsilon}} \eta^{\epsilon \in \mathrm{E}^{\epsilon}} \text { and } \\
& \mathrm{g}\left(\nabla_{\xi^{\epsilon}}^{\prime} \eta^{-\epsilon}, \nu^{-\epsilon}\right)=\epsilon 3 / 2 \mathrm{~d} \Omega\left(\xi^{\epsilon}, \eta^{-\epsilon}, \nu^{-\epsilon}\right)=0 \text {, hence } \nabla_{\xi^{\epsilon}} \eta^{-\epsilon} \in \mathrm{E}^{-\epsilon} .
\end{aligned}
$$

(One should keep in mind the property $\Omega\left(E^{\epsilon 0}, E^{\epsilon 0}\right)=0$.) It also follows from a simple computation that if $\nabla^{\prime} \mathrm{c} \equiv 0$ and $\nabla^{\prime} \mathrm{g} \equiv 0$ then $\nabla^{\prime} \Omega \equiv 0$. Therefore $\nabla^{\prime}$ satisfies (i), (ii), and (iii). 
Given arbitrary vector fields $\xi=\xi_{1}+f \dot{\varphi}$ and $\eta=\eta_{1}+\mathrm{h} \dot{\varphi}$ for real functions $\mathrm{f}$ and $\mathrm{h}$, and vector fields $\xi_{1}, \eta_{1}$ in $\mathrm{E}$, define

$$
\nabla_{\xi} \eta=\nabla_{\xi_{1}}^{\prime} \eta_{1}+\mathrm{f} \dot{\varphi}_{\dot{\varphi}} \eta+\left(\xi_{1} \mathrm{~h}\right) \dot{\varphi}
$$

It is not difficult to check that $\nabla$ so defined is the unique affine connection on $V$ that satisfies (i)-(v). Moreover, the connection $\varphi_{t}^{*} \nabla$, defined by

$$
\left(\varphi_{t}^{*} \nabla\right)_{\xi} \eta:=\mathrm{D} \varphi_{-t} \nabla_{\mathrm{D} \varphi_{t} \xi} \mathrm{D} \varphi_{t} \eta
$$

also can be shown to have the same properties. By uniqueness, we must have $\nabla=\varphi_{t}^{*} \nabla$, so that $\nabla$ is flow-invariant (note that all the objects defining $\nabla$ are $\varphi$-invariant).

By computing the Christoffel symbols of $\nabla$, one readily sees that $\nabla$ is $\mathrm{C}^{r-1}$ if the foliations are $\mathrm{C}^{r}$ (observe that the involution $\mathrm{c}$ has the same smoothness as the distributions $\mathrm{E}^{+}$and $\mathrm{E}^{-}$).

In order to prove (ii), it suffices to show that a vector field $\xi$ tangent to $\mathrm{E}^{\epsilon}$, which is invariant under the canonical map $\mathrm{H}$, satisfies $\nabla_{\eta} \xi=0$ for any vector field $\eta$ along $\mathrm{E}^{-\epsilon}$.

Let $\xi$ be a vector field defined on a neighborhood $\mathcal{W}$ of $v \in V$ such that $\xi(v) \in E^{\epsilon}(v)$ and

$$
\mathrm{H}_{w v}^{\mathcal{W}}(\mathrm{u}) \xi(\mathrm{u})=\xi\left(J \mathcal{G}_{w v}^{\mathcal{W}}(\mathrm{u})\right)
$$

for $u$ in $W$ and $w \in W^{-\epsilon 0}(v)$. Let $f$ be any differentiable function on $W$ that is constant along the leaves of $\mathrm{W}^{-\epsilon 0}$ (i.e., foJ $\mathcal{G}_{w v}=\mathrm{f}$ ). Then

$$
(\xi \mathrm{f}) \circ \mathfrak{H} \underset{w v}{W}=\xi \mathrm{f}
$$

Now, if $\eta$ is another vector field defined near $\mathrm{v}$ and tangent to $\mathrm{E}^{-\epsilon}$, we have $\eta \mathrm{f}=0$ and $\eta(\xi \mathrm{f})=0$ so that $[\xi, \eta] \mathrm{f}=0$ for every such $\mathrm{f}$. Therefore $[\xi, \eta]$ must be a vector field tangent to $E^{-\epsilon 0}$. On the other hand

$$
\nabla_{\xi} \eta-\nabla_{\eta} \xi=[\xi, \eta]-\pi^{0}[\xi, \eta] \in \mathrm{E}^{-\epsilon} \text { and } \nabla_{\xi} \eta \in \mathrm{E}^{-\epsilon} \text { and } \nabla_{\eta} \xi \in \mathrm{E}^{\epsilon}
$$


therefore $\nabla_{\eta} \xi=0$, proving the claim.

The next proposition refers to the particular case of the geodesic flow on a closed Riemannian manifold of negative curvature.

Recall the flip map $J$ and the fibration $p: \widetilde{V} \rightarrow P$, where $P$ is the space of orbits of the flow $\varphi$. Given a vector field $\xi$ on $\mathrm{P}$, denote by $\xi^{*}$ the unique flow invariant vector field on $\widetilde{V}$ such that $\xi^{*} \in \mathrm{E}=\mathrm{E}^{+} \oplus \mathrm{E}^{-}$and $\xi^{*}$ projects onto $\xi$ under Dp. We will refer to $\xi^{*}$ as the lift of $\xi$.

Proposition 8. (i) The invariant connection $\nabla$ pushes forward to an affine connection $\nabla^{p}$ on $P$, which can be characterized by: (a) $\nabla^{p} \Omega \equiv 0,(b) \nabla^{p} F^{\epsilon} \subset F^{\epsilon}$, for $\epsilon=+$ and - , and $(c) \nabla^{p}$ is torsion-free. Moreover, if $\xi$ and $\eta$ are vector fields on $P$ we have $\nabla_{\xi^{*}} \eta^{*}=\left(\nabla_{\xi}^{p} \eta\right)^{*}$.

(ii) Denote by $R$ and $R^{p}$ the curvature tensors of $\nabla$ and $\nabla^{p}$ respectively. Then $\left(\nabla^{p} R^{p}\right)_{u}=0$ if and only if $\left(\nabla_{\xi} R\right)_{v}(\eta, \zeta)=0$ for arbitrary vectors $\xi, \eta$, and $\zeta$ in $E^{+}(v) \oplus E^{-}(v)$ and any $v$ in the orbit $u \in P$.

(iii) $\nabla$ is invariant under $J$.

Proof. (i) Define an affine connection $\nabla^{p}$ on $\mathrm{P}$ by: Given vector fields $\xi$ and $\eta$ on $\mathrm{P}$,

$$
\left(\nabla_{\xi}^{p} \eta\right)^{*}=\nabla_{\xi^{*}} \eta^{*}
$$

Properties (a), (b), and (c) easily follow from the corresponding properties of $\nabla$. As an example we show that $\nabla^{p}$ is torsion-free. First we observe that, as $[\xi, \eta]^{*}$ and $\left[\xi^{*}, \eta^{*}\right]$ project onto the same vector field on $\mathrm{P}$ it follows

$$
[\xi, \eta]^{*}=\left[\xi^{*}, \eta^{*}\right]-\pi^{0}\left[\xi^{*}, \eta^{*}\right]
$$

Therefore

$$
\left(\nabla_{\xi}^{p} \eta-\nabla_{\eta}^{p} \xi-[\xi, \eta]\right)^{*}=\nabla_{\xi^{*}} \eta^{*}-\nabla_{\eta^{*}} \xi^{*}-[\xi, \eta]^{*}=
$$




$$
\begin{aligned}
& =\nabla_{\xi^{*}} \eta^{*}-\nabla_{\eta^{*}} \xi^{*}-\left(\left[\xi^{*}, \eta^{*}\right]-\pi^{0}\left[\xi^{*}, \eta^{*}\right]\right) \\
& =\mathrm{T}\left(\xi^{*}, \eta^{*}\right)+\pi^{0}\left[\xi^{*}, \eta^{*}\right] \in \mathrm{E}^{0} \\
& =0, \text { since } \pi^{0} \zeta^{*}=0 \text { for any } \zeta
\end{aligned}
$$

(ii) For vector fields $\xi, \eta$, and $\zeta$ on $\mathrm{P}$, we have

$$
\begin{aligned}
& \left(\mathrm{R}^{p}(\xi, \eta) \zeta\right)^{*}=\left(\nabla_{\xi}^{p} \nabla_{\eta}^{p} \zeta\right)^{*}-\left(\nabla_{\eta}^{p} \nabla_{\xi}^{p} \zeta\right)^{*}-\left(\nabla_{[\xi, \eta]^{p}}^{p}\right)^{*} \\
& \quad=\nabla_{\xi^{*}} \nabla_{\eta^{*}} \zeta^{*}-\nabla_{\eta^{*}} \nabla_{\xi^{*}} \zeta^{*}-\nabla_{[\xi, \eta]^{*}} \zeta^{*} \\
& \quad=\mathrm{R}\left(\xi^{*}, \eta^{*}\right) \zeta^{*}+\nabla_{\pi^{0}\left[\xi^{*}, \eta^{*}\right]^{*}} \\
& \quad=\mathrm{R}\left(\xi^{*}, \eta^{*}\right) \zeta^{*}
\end{aligned}
$$

since $\nabla_{\dot{\varphi}} \zeta^{*}=\left[\dot{\varphi}, \xi^{*}\right]=0$. It follows that

$$
\left(\left(\nabla_{\mu}^{p} \mathrm{R}^{p}\right)(\xi, \eta) \zeta\right)^{*}=\left(\nabla_{\mu^{*}} \mathrm{R}\right)\left(\xi^{*}, \eta^{*}\right) \zeta^{*},
$$

from which the claim follows (note: $\left(\nabla_{\xi_{1}} \mathrm{R}\right)\left(\xi_{2}, \xi_{3}\right) \xi_{4}=0$ if $\xi_{i} \in E_{0}$ for some i).

(iii) Follows from observing that the connection $\mathrm{J}^{*} \nabla$ defined by

$$
\left(\mathrm{J}^{*} \nabla\right) \xi^{\eta=\mathrm{DJ}} \nabla_{\mathrm{DJ}} \xi^{\mathrm{DJ} \eta}
$$

satisfies the same properties that characterize $\nabla$.

Let $g$ be the symmetric, nondegenerate bilinear form on $E$ introduced in the proof of Proposition 7 (i): $g=\Omega(\cdot, c \cdot)$, where $\mathrm{c}$ is the involution of $\mathrm{E}$ given by $\mathrm{c}=\pi^{+}-\pi^{-}$, that is, if $\xi=\xi^{+}+\xi^{-} \in \mathrm{E}^{+} \oplus \mathrm{E}^{-}$then $\mathrm{c} \xi=\xi^{+}-\xi^{-}$.

Denote by $R$ the curvature tensor of the Kanai connection $\nabla$ and consider also the tensor field of type $(0,4)$ defined by $\check{\mathrm{R}}=\mathrm{g}(\mathrm{R}(\cdot, \cdot) \cdot, \cdot)$. Its covariant derivative $\omega=\nabla \check{R}$ is a $(0,5)$-tensor field and we have $\omega \equiv 0$ if and only if $\nabla R \equiv 0$. Similarly, define $\mathrm{R}^{p}$ and $\check{\mathrm{R}}^{p}$ associated to the affine connection $\nabla^{p}$ on $\mathrm{P}$.

Proposition 9. ([FK 2] Lemma 2) (1) $R, \breve{R}$, and $\omega$ are flow-invariant tensor fields on $V$.

(2) If $\omega_{v} \neq 0$ for some $v \in V$, there exist $\epsilon \in\{+,-\}, \xi_{1}, \xi_{3}, \xi_{5} \in E^{\epsilon}(v)$ and $\xi_{2}, \xi_{4} \in E^{-\epsilon}(v)$ 
such that

$$
\omega_{v}\left(\xi_{1}, \cdots, \xi_{5}\right) \neq 0
$$

For geodesic flows, this same fact is true for $\epsilon=+$ and - .

(3) For any $v \in V, \epsilon \in\{+,-\}$ and vectors $\xi_{1}, \xi_{3}, \xi_{5} \in E^{\epsilon}(v)$ and $\xi_{2}, \xi_{4} \in E^{-\epsilon}(v)$, we have

$$
\omega_{v}\left(\xi_{1}, \cdots, \xi_{5}\right)=\omega_{v}\left(\xi_{\mu(1)}, \cdots, \xi_{\mu(5)}\right)
$$

where $\mu$ is any permutation of $\{1, \cdots, 5\}$ such that $\mu=\mu_{1} \circ \mu_{2}$ - the product of a permutation $\mu_{1}$ of $\{1,3,5\}$ and a permutation $\mu_{2}$ of $\{2,4\}$.

Proof. (1) follows naturally from the $\varphi$-invariance of $\nabla$ and g. In order to show the other properties, we need to consider the algebraic symmetries of $\omega$. First, let us observe that $\check{\mathrm{R}}_{v}\left(\xi_{1}, \xi_{2}, \xi_{3}, \xi_{4}\right)=0$ whenever $\xi_{1}$ and $\xi_{2}$, or $\xi_{3}$ and $\xi_{4}$ belong to the same subbundle $\mathrm{E}^{\epsilon 0}$ for $\epsilon=+$ or - . In fact, as $\mathrm{R}\left(\xi_{1}, \xi_{2}\right) \mathrm{E}^{\epsilon 0} \subset \mathrm{E}^{\epsilon 0}$ and $\Omega\left(\mathrm{E}^{\epsilon 0}, \mathrm{E}^{\epsilon 0}\right)=0$, we have $\check{\mathrm{R}}_{v}\left(\xi_{1}, \ldots, \xi_{4}\right)=\Omega\left(\mathrm{R}_{v}\left(\xi_{1}, \xi_{2}\right) \xi_{3}, \xi_{4}\right)=0$, whenever $\xi_{3}$ and $\xi_{4}$ belong to the same space $\mathrm{E}^{\epsilon}$. On the other hand, it is well known (see, e.g., [He]) that the curvature tensor of an affine connection associated to an indefinite metric satisfies

$$
\check{\mathrm{R}}_{v}\left(\xi_{1}, \xi_{2}, \xi_{3}, \xi_{4}\right)=\check{\mathrm{R}}\left(\xi_{3}, \xi_{4}, \xi_{1}, \xi_{2}\right)
$$

so that the same property holds for the first pair.

$\check{\mathrm{R}}$ also satisfies the following symmetries, true for any curvature tensor with an indefinite metric (see, e.g., [He]). We use the abbreviation

$$
\left(\begin{array}{llll}
1 & 2 & 3 & 4
\end{array}\right):=\check{\mathrm{R}}\left(\xi_{1}, \xi_{2}, \xi_{3}, \xi_{4}\right) \text {. }
$$

Then one has

$$
\left(\begin{array}{llll}
1 & 2 & 3 & 4
\end{array}\right)=\left(\begin{array}{llll}
3 & 4 & 1 & 2
\end{array}\right)=-\left(\begin{array}{llll}
2 & 1 & 3 & 4
\end{array}\right)=-\left(\begin{array}{llll}
1 & 2 & 4 & 3
\end{array}\right)
$$

$$
\left(\begin{array}{llll}
1 & 2 & 3 & 4
\end{array}\right)+\left(\begin{array}{llll}
2 & 3 & 1 & 4
\end{array}\right)+\left(\begin{array}{llll}
3 & 1 & 2 & 4
\end{array}\right)=0 \text { (first Branchi identity). }
$$

From these properties and the second Bianchi identity ((vi) below) we obtain, for

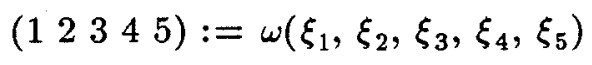




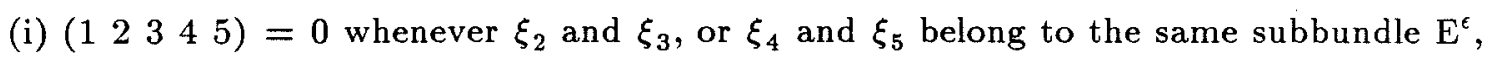
$\epsilon=+$ or -

(ii)

$\left(\begin{array}{lllll}1 & 2 & 3 & 4 & 5\end{array}\right)=-\left(\begin{array}{lllll}1 & 3 & 2 & 4 & 5\end{array}\right)$

(iii)

$\left(\begin{array}{lllll}1 & 2 & 3 & 4 & 5\end{array}\right)=-\left(\begin{array}{lllll}1 & 2 & 3 & 5 & 4\end{array}\right)$

(iv)

$$
\left(\begin{array}{lllll}
1 & 2 & 3 & 4 & 5
\end{array}\right)=\left(\begin{array}{lllll}
1 & 4 & 5 & 2 & 3
\end{array}\right)
$$

(v)

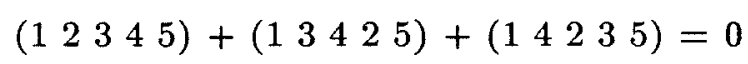

(vi)

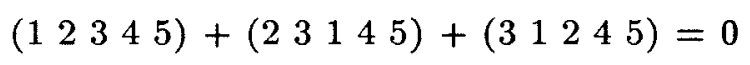

The identities (i) - (v) are easily obtained from the corresponding properties of $\check{\mathrm{R}}$ by using that $\nabla E^{\epsilon} \subset E^{\epsilon}$ and the formula

$$
\omega_{v}\left(\xi_{1}, \ldots, \xi_{5}\right)=\xi_{1} \check{\mathrm{R}}\left(\tilde{\xi}_{2}, \ldots, \tilde{\xi}_{5}\right)-\sum_{i=2}^{5} \check{\mathrm{R}}\left(\xi_{2}, \ldots, \nabla_{\xi_{1}} \tilde{\xi}_{i}, \ldots, \xi_{5}\right),
$$

where $\xi_{i} \in \mathrm{E}^{\delta}(\mathrm{v})$ and $\tilde{\xi}_{\mathrm{i}}$ is any smooth vector field in $\mathrm{E}^{\delta}$ that extends $\xi_{i}$ near $\mathrm{v}$. It is now immediate to show that (i) - (vi) imply (2) and (3). 


\section{An Outline of Kanai's Work}

In this section we indicate how the conclusion of the Theorem follows after we establish $\nabla R \equiv 0$, where $R$ is the curvature tensor associated to the invariant affine connection $\nabla$ (which is a $\mathrm{C}^{\infty}$ connection, since we are assuming the Anosov foliations are $\mathrm{C}^{\infty}$ ). This constitutes the bulk of $[\mathrm{K}]$ and the reader may wish to consult that paper for the details.

Let us first look at the model space $\mathbb{R H}^{n}$ - the real hyperbolic space - for inspiration. This space can be represented as the homogeneous manifold

$$
\mathbb{R H}^{n}=\mathrm{SO}_{0}(\mathrm{n}, 1) / \mathrm{SO}(\mathrm{n}, \mathbb{R}),
$$

where $\mathrm{G}=\mathrm{SO}_{0}(\mathrm{n}, 1)$ is the connected component of the identity of the orthogonal group associated to the quadratic form $\mathrm{Q}(\mathrm{x})=\mathrm{x}_{1}^{2}+\cdots+\mathrm{x}_{n}^{2}-\mathrm{x}_{n+1}^{2}$ and $\mathrm{K}=\mathrm{SO}(\mathrm{n}, \mathbb{R})$ is its maximal compact subgroup. The unit tangent bundle $\hat{V}$ of $\mathbb{R} H^{n}$ is given by

$$
\hat{\mathrm{V}}=\mathrm{SO}_{0}(\mathbf{n}, 1) / \mathrm{SO}(\mathbf{n}-1, \mathbf{R}) \text {. }
$$

In order to describe the geodesic flow on $\hat{\mathrm{V}}$, we observe that the Lie algebra $\mathfrak{F}$ of $\mathrm{G}$ can be shown to decompose as the direct sum

$$
\mathfrak{G}=\Re_{-1}+\mathfrak{\beta}_{0}+\Re_{1}
$$

which defines the structure of a graded Lie algebra, i. e., if we set $\Re_{k}=0$ for $k \neq-1,0$, or 1 , then

$$
\left[\mathfrak{P}_{i}, \Re_{j}\right] \subset \mathfrak{B}_{i+j}, \text { for } \mathrm{i}, \mathrm{j} \in\{-1,0,1\}
$$

and $\Re_{0}$ is a subalgebra isomorphic to $\mathbb{R} \cdot \delta \oplus \operatorname{so}(n-1, \mathbb{R})=\mathbb{R} \cdot \delta \oplus \mathfrak{S}_{0}$. Here, $\delta$ is a vector that generates the center of $\mathfrak{P}_{0}$. The adjoint action of $\delta$ on $\mathfrak{G}$ satisfies

$$
\left.\operatorname{ad}(\delta)\right|_{\mathfrak{P}_{j}}=\mathrm{j} \cdot \mathrm{I}_{\mathfrak{P}_{j}}, \text { for } \mathrm{j} \in\{-1,0,1\}
$$

We can integrate $\delta$ to obtain a flow on $\hat{\mathrm{V}}$ as follows. Let $\mathrm{g}_{t}=\operatorname{Exp} \mathrm{t} \delta$ (Exp denotes the exponential map from $\mathfrak{F}$ to $G$ ) and consider the flow on $G$ defined by right 
multiplication by $\mathrm{g}_{t}$. Since $\mathrm{g}_{-t} \mathrm{Hg}_{t}=\mathrm{H}$ ( $\mathrm{H}$ being the analytic subgroup of $\mathrm{G}$ with Lie algebra $\operatorname{so}(n-1, \mathbb{R})), \mathrm{g}_{t}$ defines a flow on the quotient space - the geodesic flow of $\mathbb{R} H^{n}$ :

$$
\hat{\varphi}_{t}: \hat{\mathrm{V}}=\mathrm{G} / \mathrm{H} \rightarrow \mathrm{G} / \mathrm{H} \text {. }
$$

The space of orbits $\hat{P}$ can be given as $\hat{P}=G / H_{0}$, where

$$
\mathrm{H}_{0} \simeq\{\mathrm{s} \cdot \mathrm{g}: \mathrm{g} \in \mathrm{SO}(\mathrm{n}-1, \mathbb{R}), \mathrm{s}>0\}
$$

is the closed analytic subgroup of $G$ with Lie algebra $\Re_{0}$.

The linear subspaces $\mathbb{P}_{-1}$ and $\mathfrak{P}_{1}$, considered as G-invariant distributions of linear subspaces in TG, define integrable distributions in the quotient spaces $\hat{\mathrm{V}}$ and $\hat{\mathrm{P}}$. The corresponding invariant foliations are the Anosov foliations in $\hat{V}$ and its projections on $\hat{\mathrm{P}}$. We will continue to use for these objects the same notations as in the previous paragraphs except that we will add a " $"$ to indicate that the object is associated to the model (homogeneous) space. So, for example, $\hat{W}^{+0}$ denotes the strong unstable foliation in $\hat{\mathrm{V}}$.

The space of leaves of $\hat{W}^{\epsilon 0}, \hat{\mathrm{B}}=\hat{\mathrm{B}}^{\epsilon}=\hat{\mathrm{V}} / \hat{\mathrm{W}}^{\epsilon 0}(\epsilon=+,-)$ can also be given as a homogeneous space: $B \simeq G / \mathrm{H}^{+}$, where $\mathrm{H}^{+}$is the closed analytic subgroup of $\mathrm{G}$ with Lie algebra $\beta_{0}+\Re_{1}$ and we can identify $B$ with the $n-1$-sphere

$$
\mathrm{S}^{n-1}=\left\{\mathrm{x} \in \mathbb{R}^{n, 1}: \mathrm{x}_{n+1}=1, \mathrm{x}_{1}^{2}+\cdots+\mathrm{x}_{n}^{2}-\mathrm{x}_{n+1}^{2}=0\right\}
$$

The action of $G$ on $\hat{B}$ preserves the conformal structure of $S^{n-1}$ induced from the Minkowski space $\mathbb{R}^{n, 1}$.

$\hat{\mathbf{P}}$ is a symplectic manifold. The (G-invariant) symplectic form $\hat{\Omega}$ can be defined as follows. Let $\delta^{*}$ denote the dual form of $\delta$, so that $\delta^{*}(\delta)=1, \delta^{*}\left(\mathfrak{P}_{-1}+\mathfrak{S}_{0}+\mathfrak{P}_{1}\right)=0$. $\delta^{*}$ may be viewed as a $\mathrm{G}$-invariant 1 -form on $\mathrm{G}$. It can be shown that $\delta^{*}$ descends to the quotient $\hat{\mathrm{V}}$ and defines a contact form $\hat{\theta}$ there. The exterior derivative $\mathrm{d} \delta^{*}$ descends further down to $\hat{P}$ and defines the symplectic form $\hat{\Omega}$ there. 
The homogeneous space $\hat{\mathrm{P}}$ is, in fact, an affine symmetric space. The Ginvariant affine connection $\hat{\nabla}^{p}$ on $\hat{\mathrm{P}}$ is exactly the Kanai connection defined in the previous section. It is a complete torsion-free connection such that $\hat{\nabla}^{p} \hat{\mathrm{R}}^{p} \equiv 0\left(\hat{\mathrm{R}}^{p}\right.$ is the corresponding curvature tensor).

Let us now consider the geodesic flow $\varphi$ in the unit tangent bundle $V$ of a compact manifold $M$ of dimension $n \geq 3$ and negative curvature. Also suppose the Anosov foliations are $\mathrm{C}^{\infty}$ so that the affine connection $\nabla^{p}$ on $\mathrm{P}$ defined in the previous section is also $\mathrm{C}^{\infty}$. Assume that $\nabla^{p} \mathrm{R}^{p} \equiv 0$, i.e., $\left(\mathrm{P}, \nabla^{p}\right)$ is an affine locally-symmetric space.

The first step of Kanai's proof is to understand the structure of the group of affine transformations of $\nabla^{p}$. The Lie algebra of this group can be described as follows:

Let $x \in P$ and define $\Re_{\epsilon 1}=F^{\epsilon}(x), \epsilon=+$ or - . Define $\Re_{0}$ as the space of endomorphisms $\alpha$ of $\Re_{\beta}=\Re_{-1}+\Re_{1}$ such that $\alpha \Re_{\epsilon} \subset \Re_{\epsilon}(\epsilon=+$ and -$)$ and $\alpha \cdot R^{p} \equiv 0(\alpha$ acts on the tensor algebra of $\mathrm{T}_{x} \mathrm{P}$ as a derivation). It follows from $\nabla^{p} \mathrm{R}^{p} \equiv 0$ and the Bianchi identity for $\nabla^{p}$ that for all $\xi, \eta \in \mathrm{T}_{x} \mathrm{P}, \mathrm{R}^{p}(\xi, \eta) \in \Re_{0}$ and

$$
\mathfrak{G}=\mathfrak{P}_{-1}+\mathfrak{P}_{0}+\mathfrak{P}_{1}
$$

is a real finite dimensional Lie algebra with the brackets defined as: Given $\alpha, \beta \in \mathfrak{P}_{0}$ and $\xi, \eta \in \mathrm{T}_{x} \mathrm{P}$

$$
[\alpha, \beta]=\alpha \circ \beta-\beta \circ \alpha,[\alpha, \xi]=\alpha \xi,[\xi, \eta]=-\mathrm{R}^{p}(\xi, \eta)
$$

It is easy to show that $\mathfrak{G}$ satisfies the property $(*)$ and it contains an element $\delta$ satisfying (**). Moreover $\mathfrak{P}_{0}$ acts faithfully on $\mathfrak{P}_{\epsilon}$, i.e., if $\alpha \in \mathfrak{P}_{0}$ and $\left[\alpha, \mathfrak{\beta}_{\epsilon}\right]=0$, then $\alpha=0$. A Lie algebra satisfying these properties is called in $[\mathrm{K}]$ a bipolarized symmetric Lie algebra. 
Denote by $\hat{P}$ the homogeneous space $\hat{P}=G / H$, where $G$ is the analytic real Lie group with Lie algebra $\mathfrak{G}$ and $\mathrm{H}$ is the connected analytic subgroup of $\mathrm{G}$ whose Lie algebra is $\beta_{0} . \quad H$ is a closed subgroup since it is the set of fixed points of an involution of $\mathrm{G}$ that integrates the Lie algebra automorphism $\sigma: \mathfrak{G} \rightarrow \mathfrak{G}$, defined by $\sigma \xi=\xi$ for $\xi \in \mathfrak{P}_{0}$ and $\sigma \xi=-\xi$ for $\xi \in \Re$.

The linear subspaces $\Re_{-1}$ and $\Re_{1}$, considered as left-invariant distributions on $\hat{\mathrm{P}}$ are integrable and we denote the corresponding (G-invariant) foliations by $\hat{\mathfrak{F}}^{+}$and $\hat{\mathfrak{F}}^{-}$. Let $\hat{\nabla}$ denote the canonical G-invariant affine connection on $\hat{\mathrm{P}}$, which makes $\mathrm{P}$ and $\hat{\mathrm{P}}$ locally isomorphic as affine spaces (see $[\mathrm{KN}])$.

Next step in Kanai's argument is the classification of the Lie algebras $\mathfrak{5}$ that can occur. This is based on the classification of graded Lie algebras of the first kind (i.e., satisfying $(*)$ and $\Re_{k}=0$ for $|k| \geq 2$ ) obtained by Kobayashi and Nagano in [KNa], and on the following lemma.

Denote by $\mathrm{H}^{\epsilon}, \epsilon=+$, or - , the connected analytic subgroups of $\mathrm{G}$ corresponding to the Lie subalgebras $\mathfrak{S}^{\epsilon}=\mathfrak{B}_{0}+\mathfrak{P}_{\epsilon}$. It is shown in $[K]$ that $H^{\epsilon}$ are closed subgroups. Define $\hat{B}=G / H^{\epsilon}$. Then

Lemma ((3.3) and (3.6) in [K]). If $B^{+}$and $B^{-}$are compact, then $\mathfrak{G}$ is semisimple. If this is the case, the universal coverings of $B^{+}$and $B^{-}$are diffeomorphic to a certain compact Riemannian symmetric space $B_{0}$. Moreover, if $B_{0}$ is irreducible (i. e., if it does not admit a nontrivial decomposition $B_{0}=B_{1} \times \cdots \times B_{r}, r>1$ ), then $\mathfrak{f}$ is simple.

In section 4.1 of $[\mathrm{K}]$, it is shown that $\mathrm{B}_{0}$ is diffeomorphic to a sphere that is identified with the ideal boundary of $\tilde{\mathbf{M}}$. Therefore, 5 must be a simple Lie algebra and 
[KNa] yields that $\mathfrak{G} \simeq \operatorname{sl}(\mathrm{n}, \mathbb{R})$ or $\mathrm{so}(\mathrm{n}, 1)$. The wrong algebra $\mathrm{sl}(\mathrm{n}, \mathbb{R})$ can be discarded by using certain properties of the action of $\Gamma$ on the ideal boundary of $\tilde{M}([K] 4.1)$.

Therefore we have, as for the real hyperbolic space, $\hat{\mathrm{P}}=\mathrm{SO}_{0}(\mathrm{n}, 1) / \mathrm{H}^{0}$, where $\mathrm{H}^{0}$ is defined by $\mathrm{H}^{0} \simeq\{\mathrm{s} \cdot \mathrm{g}: \mathrm{g} \in \mathrm{SO}(\mathrm{n}-1, \mathbb{R}), \mathrm{s}>0\}$. Moreover one has $([\mathrm{K}], \mathrm{p}, 22)$ :

$$
\operatorname{Aut}(\hat{\mathrm{P}}):=\operatorname{Aut}\left(\hat{\mathrm{P}}, \hat{\Omega}, \hat{\mathfrak{F}}^{+}, \hat{\mathfrak{F}}^{-}\right)
$$

$:=$ group of diffeomorphisms of $\hat{\mathrm{P}}$ that preserve $\hat{\Omega}$ and the foliations $\mathcal{F}^{\epsilon}$

$\simeq$ group of isometries of $\mathbb{R H}{ }^{n}$

It is shown in section 4.2 of $[\mathrm{K}]$ that the developing map $\Psi: \mathrm{P} \rightarrow \hat{\mathrm{P}}$ (obtained from the fact that $\left(\mathrm{P}, \nabla^{p}\right)$ and $\left(\hat{\mathrm{P}}, \hat{\nabla}^{p}\right)$ are locally isomorphic) is a diffeomorphism that preserves the Anosov foliations.

The next crucial point in the proof is the observation that, under the affine diffeomorphism $\Psi$, the symplectic form $\Omega$ on $\mathrm{P}$ is pushed forward to a multiple of $\hat{\Omega}$. After a homothetic change of the metric on the hyperbolic space, we can assume that $\left(\Psi^{-1}\right)^{*} \Omega=\hat{\Omega}$.

The fact that $\Psi$ is now a symplectic diffeomorphism allows one to define a transitive action of $\mathrm{G}$ on $\tilde{\mathrm{V}}$ commuting with $\varphi$. This is explained in section 4.4 of [K].

From that, one obtains a diffeomorphism $\Phi: \tilde{V} \rightarrow \hat{V}$ such that $\Phi \circ \varphi_{t}=\hat{\varphi}_{\tau} \circ \Phi$. After deforming $\Phi$ by adding a global shift (i.e., after replacing $\Phi$ by $\hat{\varphi}_{r} \circ \Phi$ for some $\tau \in \mathbb{R}$ ), one obtains a diffeomorphism between $\tilde{V}$ and $\hat{V}$, which is $\Gamma$-equivariant $(\Gamma-$ the fundamental group of $M$ - acts on $\hat{V}$ since $\left.\Gamma \subset \operatorname{Aut}(P)=\operatorname{Aut}(\hat{P}) \simeq \operatorname{Isom}\left(\mathbb{R} H^{n}\right)\right)$. This lets us define the required diffeomorphism between the compact manifolds $V=\Gamma \backslash \tilde{V}$ and $\Phi \Gamma \Phi^{-1} \backslash \hat{\mathrm{V}}$ 


\section{Some Smooth Ergodic Theory}

Let $\mathrm{V}$ be a compact $\mathrm{C}^{\infty}$ Riemannian manifold and $\|\cdot\|$ the corresponding norm on TV. Let $\varphi_{t}: \mathrm{V} \rightarrow \mathrm{V}$. denote a $\mathrm{C}^{\infty}$ flow defined on $\mathrm{V}$. If $\mathrm{v} \in \mathrm{V}$ and $\xi \in \mathrm{T}_{v} \mathrm{~V}$, define

$$
\chi^{ \pm}(\mathrm{v}, \xi):=\limsup _{t \rightarrow \pm \infty} \frac{1}{\mathrm{t}} \log \left\|\left(\mathrm{D} \varphi_{t}\right)_{v} \xi\right\|
$$

For each $v \in V, \chi^{+}(v, \cdot)$ assumes finitely many values on $T_{v} V$, say

$$
\chi_{1}(\mathrm{v})<\chi_{2}(\mathrm{v})<\cdots<\chi_{s(v)}(\mathrm{v}), \mathrm{s}(\mathrm{v}) \leq \operatorname{dim} \mathrm{T}_{v} \mathrm{~V}
$$

Define

$$
\mathrm{F}_{i}(\mathrm{v})=\left\{\xi \in \mathrm{T}_{v} \mathrm{~V}: \chi^{+}(\mathrm{v}, \xi) \leq \chi_{i}(\mathrm{v})\right\}
$$

$F_{i}(v)$ is a linear subspace of $T_{v} V$, and we have the filtration:

$$
\{0\}=\mathrm{F}_{0}(\mathrm{v}) \subset \mathrm{F}_{1}(\mathrm{v}) \subset \cdots \subset \mathrm{F}_{s(v)}(\mathrm{v})=\mathrm{T} v \mathrm{~V} .
$$

The functions $\chi_{i}, \mathrm{~s}$, and the filtration $\left(\mathrm{F}_{i}\right)$ are $\varphi_{t}$-invariant and measurable, as functions of $v \in V$.

The following proposition is a reduced version, enough for our needs, of the Multiplicative Ergodic Theorem of Oseledec (see, e.g., [P] or [M]).

Let $\lambda$ be any $\varphi_{t}$-invariant Borel probability measure on $\mathrm{V}$. The set $\Lambda$ defined in the next proposition is called the set of Lyapunov regular points.

Proposition 9. Let $V$ be a compact $C^{\infty}$ Riemannian manifold, $\varphi_{t}: V \rightarrow V a$ smooth flow on $V$, and $\lambda$ a $\varphi$-invariant Borel probability measure on $V$. Then there exists a $\varphi$-invariant measurable set $\Lambda \subset V$ of full Lebesgue measure with the following property: For each $v \in \Lambda$, there are linear subspaces $E_{j}(v)$ of $T_{v} V$ such that

$$
T_{v} V=\underset{j=1}{\stackrel{s(v)}{\oplus}} E_{j}(v)
$$

and

$$
\chi^{ \pm}(v, \xi)=\lim _{t \rightarrow \pm \infty} \frac{1}{\bar{t}} \log \left\|\left(D \varphi_{t}\right)_{v} \xi\right\|=\chi_{j}(v)
$$


uniformily in $\xi \in E_{j}(v)$. The subspaces $E_{j}(v), j=1, \cdots, s(v)$, and the functions $s$, $\chi_{1}, \cdots, \chi_{s}$ depend measurably on $v$ and are $\varphi$-invariant.

Assume now that $\varphi$ is a contact Anosov flow. We have therefore the hyperbolic decomposition $E=E^{+} \oplus E^{-}$, where $E$ is the kernel of the contact form $\theta$ and $\Omega=\left.d \theta\right|_{E}$ defines a symplectic form. In this case we may also consider the filtration

$$
\{0\}=\mathrm{F}_{0}^{+} \subset \mathrm{F}_{1}^{+} \subset \cdots \subset \mathrm{F}_{r}^{+}=\mathrm{E}^{+}
$$

where $\mathrm{r}=(\mathrm{s}-1) / 2$ and $\mathrm{F}_{i+r+1}(\mathrm{v})=\mathrm{F}_{i}^{+}(\mathrm{v}) \oplus \mathrm{E}^{-}(\mathrm{v})$.

The following proposition says that the holonomy transport of vectors in $\mathrm{E}^{+}$ along $\mathrm{W}^{-0}$ preserves the filtration $\left(\mathrm{F}_{i}^{+}\right)$and the forward exponents $\chi_{i}^{+}$.

Proposition 10. ([FK2] Lemma 3). Assume the Anosov splitting $T V=E^{+} \oplus E^{-} \oplus E^{0}$ is differentiable of class $C^{r}, r \geq 1$. Let $v, w \in V$, with $v \in W^{-0}(w)$. Then

$$
s(v)=s(w), \chi_{i}^{+}(v)=\chi_{i}^{+}(w)
$$

for $i=1, \cdots, s$, and for each $i$

$$
H_{w v}(v) F_{i}^{+}(v)=F_{i}^{+}(w) .
$$

In particular, the filtration $\left(\mathrm{F}_{i}^{+}\right)$is $C^{r-1}$ along the leaves of $W^{-0}$.

Proof. It suffices to prove that, if $v \in \mathrm{W}^{-}(\mathrm{w}), \xi \in \mathrm{F}_{i}^{+}(\mathrm{v})$, and $\xi^{\prime}:=\mathrm{H}_{w v}(\mathrm{v}) \xi$, then $\chi^{+}\left(\mathrm{w}, \xi^{\prime}\right)=\chi^{+}(\mathrm{v}, \xi)$. This is in fact true since

$$
\begin{gathered}
\chi^{+}\left(\mathrm{w}, \xi^{\prime}\right)=\limsup _{t \rightarrow+\infty} \frac{1}{\mathrm{t}} \log \left\|\mathrm{D} \varphi_{t} \mathrm{H}_{w v}(\mathrm{v}) \xi\right\| \\
=\limsup _{t \rightarrow+\infty} \frac{1}{\mathrm{t}} \log \left\|\mathrm{H}_{\varphi_{t}(w) \varphi_{t}(v)} \mathrm{D} \varphi_{t} \xi\right\|
\end{gathered}
$$

But

$$
\limsup _{t \rightarrow+\infty} \operatorname{dist}\left(\varphi_{t}(\mathrm{w}), \varphi_{t}(\mathrm{v})\right)=0
$$

so that there are constants $0<\mathrm{c}, \mathrm{c}^{\prime}$ such that for every $\mathrm{t} \geq 0$ and $\eta \in \mathrm{E}^{+}\left(\varphi_{t}(\mathrm{v})\right)$,

$$
\mathrm{c}^{\prime}\|\eta\| \leq\left\|\mathrm{H}_{\varphi_{t}(w) \varphi_{t}(v)} \eta\right\| \leq \mathrm{c}\|\eta\| .
$$


Therefore

$$
\chi^{+}\left(\mathrm{w}, \xi^{\prime}\right)=\limsup _{t \rightarrow+\infty} \frac{1}{\mathrm{t}} \log \left\|\mathrm{D} \varphi_{t} \xi\right\|=\chi^{+}(\mathrm{v}, \xi)
$$

Invariant Tensor Fields and Resonance Relations for the Lyapunov Exponents. We continue to consider a $\mathrm{C}^{\infty}$ contact Anosov flow $\varphi_{t}: \mathrm{V} \rightarrow \mathrm{V}, \mathrm{t} \in \mathbb{R}$, on a compact $\mathrm{C}^{\infty}$ manifold V.

Define the set $\mathrm{C}_{i n v}^{l}\left(\stackrel{r}{\otimes} \mathrm{T}^{*} \mathrm{~V}\right)$ of all flow-invariant $\mathrm{C}^{r}$ tensor fields on $\mathrm{V}$ of type $(0, \mathrm{r})$. We will also need to consider the set $\mathrm{C}_{i n v}^{l}\left(\stackrel{r}{\otimes} \mathrm{E}^{*}\right)$ of tensor fields $\tau$ contained in $\mathrm{C}_{i n v}^{l}\left(\stackrel{\nabla}{*} \mathrm{~T}^{*} \mathrm{~V}\right)$, which vanish when contracted with vectors in $\mathrm{E}^{0}$; that is, if $\xi_{1}, \cdots, \xi_{r}$ are vectors in $\mathrm{T}_{v} \mathrm{~V}$, then

$$
\tau\left(\xi_{1}, \cdots, \xi_{r}\right)=0
$$

if at least one $\xi_{i}$ belongs to $\mathrm{E}^{0}(\mathrm{v})$.

We note that there is no loss of generality in considering only tensor fields of type $(0, r)$. This is because we have a duality between $T V$ and $T^{*} V$ given by means of the $\varphi$-invariant, nondegenerate bilinear form

$$
\theta \otimes \theta+\Omega
$$

Thereby we obtain a correspondence between $(1, m)$ and $(0,1+m)$-tensors preserving $\varphi$ invariance. Moreover, since the above bilinear form is parallel with respect to the invariant affine connection defined in the previous section, that correspondence also sends parallel tensors into parallel tensors.

The following proposition shows that for a nontrivial continuous tensor field on $\mathrm{V}$, invariant under the flow $\varphi$, the Lyapunov exponents of $\varphi$ must satisfy an arithmetic condition. We call this condition a resonance relation.

Proposition 11 ([FK1] Lemma 1). Let $\tau \in C_{i n v}^{0}\left(\stackrel{r}{\otimes} T^{*} V\right)$ and $v \in \Lambda$ - the set of 
Lyapunov regular points - be such that $\tau_{v} \neq 0$. Consider vectors $\xi_{i} \in E_{l_{i}}(v), i=1, \ldots, r$, such that

$$
\tau\left(\xi_{1}, \ldots, \xi_{r}\right) \neq 0
$$

Then

$$
\sum_{i=1}^{r} \chi_{l_{i}}=0
$$

Proof. We have

$$
0 \neq \tau_{v}\left(\xi_{1}, \ldots, \xi_{r}\right)=\left(\varphi_{t}^{*} \tau\right)_{v}\left(\xi_{1}, \ldots, \xi_{r}\right)=\tau_{\varphi_{t}(v)}\left(\left(\mathrm{D} \varphi_{t}\right)_{v} \xi_{1}, \ldots,\left(\mathrm{D} \varphi_{t}\right)_{v} \xi_{r}\right)
$$

But $\left\|\tau_{v}\right\|$ is bounded for all $\mathrm{v} \in \mathrm{V}$, so that

$$
0<\left|\tau_{v}\left(\xi_{1}, \ldots, \xi_{r}\right)\right| \leq \mathrm{c} \cdot\left\|\left(\mathrm{D} \varphi_{t}\right)_{v} \xi_{1}\right\| \ldots\left\|\left(\mathrm{D} \varphi_{t}\right)_{v} \xi_{r}\right\|
$$

for some constant $c>0$ and

$$
\frac{\log \left|\tau_{v}\left(\xi_{1}, \cdots, \xi_{r}\right)\right|}{|\mathrm{t}|} \leq \frac{\log \mathbf{c}}{|\mathrm{t}|}+\frac{\mathrm{t}}{|\mathrm{t}|} \sum_{i=1}^{r} \frac{\log \left\|\left(\mathrm{D} \varphi_{t}\right)_{v} \xi_{i}\right\|}{\mathrm{t}}
$$

Passing to the limit as $\mathrm{t} \rightarrow+\infty$, and $\mathrm{t} \rightarrow-\infty$, we obtain $0 \leq \pm \sum_{i=1}^{r} \chi_{l_{i}}$, so that $\sum_{i=1}^{r} \chi_{l_{i}}=0$

For the following lemma, let $\nabla$ denote any $\varphi$-invariant smooth affine connection on $\mathrm{V}$, where $\varphi_{t}: \mathrm{V} \rightarrow \mathrm{V}$ is a smooth Anosov flow on the compact smooth manifold $\mathrm{V}$, preserving a smooth probability measure $\lambda$. We require that $\nabla_{\dot{\varphi}}$ be the Lie derivative along the flow.

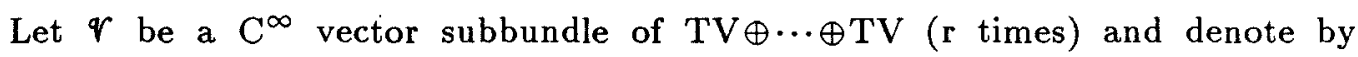
$\rho: \checkmark \rightarrow \mathrm{V}$ the base point projection. We will regard a tensor field $\tau \in \mathrm{C}_{i n v}^{\infty}\left({ }^{r} \mathrm{~T}^{*} \mathrm{~V}\right)$ as a real valued function on $\checkmark$ :

$$
\xi=\left(\xi_{1}, \cdots, \xi_{r}\right) \in q_{v}=\rho^{-1}(v) \mapsto \tau_{v}\left(\xi_{1}, \cdots, \xi_{r}\right) \in \mathbb{R}
$$

If $\xi_{0} \in \mathrm{T}_{v} \mathrm{~V}, \mathrm{v} \in \mathrm{V}$ and $\xi \in \rho^{-1}(\mathrm{v})$, let us define 


$$
\omega\left(\xi_{0}, \xi\right)=\left(\nabla_{\xi_{0}} \tau\right)(\xi)
$$

Given $\mathrm{X} \in \mathrm{T}_{\xi}{ }^{q}, \xi \in \mathbb{q}$, consider the directional derivative $(\mathrm{D} \tau)_{\xi} \mathrm{X}$ of $\tau$ along the vector $\mathrm{X}$.

Lemma 3. Let $\tau \in C_{i n v}^{\infty}\left(\stackrel{r}{\otimes} T^{*} V\right)$ and denote by $\Lambda^{\prime}$ the subset of $\Lambda$ consisting of points $v$ with the following property: There exist $\xi_{0} \in T_{v} V$ and $\xi=\left(\xi_{1}, \cdots, \xi_{r}\right) \in \rho^{-1}(v)$ such that

$$
\xi_{i} \in E_{l_{i}}(v), \text { for } i=1, \cdots, r
$$

and for every $X \in T_{v} q$ for which $(D \rho)_{\xi} X=\xi_{0}$ we have

$$
(D \tau)_{\xi} X=\omega_{v}\left(\xi_{0}, \xi\right) \neq 0 .
$$

Then $\Lambda^{\prime}$ is a set of measure zero.

Proof. If $\Lambda^{\prime}=\emptyset$ there is nothing to prove. Otherwise, pick $v \in \Lambda^{\prime}$ and $\xi_{0} \in T_{v} V$, $\xi=\left(\xi_{1}, \cdots, \xi_{r}\right) \in \rho^{-1}(\mathrm{v})$ as in the statement of the lemma.

We claim that $\tau_{v}(\xi)=0$. If not, it would follow by Proposition 11 that

$$
\sum_{i=1}^{r} \chi_{l_{i}}(v)=0
$$

But $\omega_{v}\left(\xi_{0}, \xi\right) \neq 0$, so by the same proposition

$$
\sum_{i=0}^{r} \chi_{l_{i}}(v)=0 \text {. }
$$

Hence $\chi_{l_{0}}(\mathrm{v})=0$. This implies that $\xi_{0}=\mathrm{a} \cdot \dot{\varphi}$. But then $\nabla_{\xi_{0}} \tau=\mathrm{a} \cdot £_{\dot{\varphi}} \tau=0$, since $\tau$ is $\varphi$ invariant ( $£$ denotes the Lie derivative). This contradiction proves the claim.

Consider now the level set of $\tau$

$$
\mathcal{N}=\{\eta \in \mathscr{V}: \tau(\eta)=0\}
$$

Since $(D \tau)_{\xi} \neq 0$ for the $\xi$ chosen above, the Implicit Function Theorem implies the existence of a neighborhood $\mathcal{U}_{\xi}$ of $\xi \in \mathbb{V}$ such that $\mathcal{N} \cap \mathcal{U}_{\xi}$ is a smooth (connected) submanifold embedded in $r$. 
We note that for every $\mathrm{Y} \in \mathrm{T}_{\xi^{\mathcal{N}}} \mathcal{N},(\mathrm{D} \tau)_{\xi} \mathrm{Y}=0$ so that there is no $\mathrm{Y} \in \mathrm{T}_{\xi} \mathcal{N}$ for which $(D \rho)_{\xi} \mathrm{Y}=\xi_{0}$. Therefore $\xi$ is a critical point of the smooth function $\left.\rho\right|_{\mathcal{N} \cap \mathcal{U}_{\xi}}$ (of course $\left.\operatorname{dim} \mathcal{N} \cap \mathcal{U}_{\xi}>\operatorname{dim} V\right)$.

Denote by $\mathcal{N}^{\prime}$ the smooth submanifold of $\mathscr{r}$ consisting of all $\xi$ in $\mathcal{N}$ for which there exists a neighborhood $\mathcal{U}$ of $\xi$ in $q$ such that $\mathcal{N} \cap \mathcal{U}$ is a smooth manifold embedded in $\mathscr{\gamma} . \mathcal{N}^{\prime}$ contains all the neighborhoods $\mathcal{N} \cap \mathcal{U}_{\xi}$ just constructed. Therefore the restriction $\left.\rho\right|_{\mathcal{N}^{\prime}}$ is a smooth function of which all the points of $\Lambda^{\prime}$ are critical values. By Sard's Theorem $\lambda\left(\Lambda^{\prime}\right)=0$.

Let $\Omega$ be a smooth invariant symplectic form defined on $\mathrm{E}^{+} \oplus \mathrm{E}^{-}$. Due to Proposition 11, applied to the invariant form $\Omega$, if $\chi$ is a Lyapunov exponent of $\varphi$, then so is $-\chi$. If $\mathrm{E}_{\chi}$ and $\mathrm{E}_{-\chi}$ denote the corresponding subspaces in the Oseledec decomposition, then it also follows that $\Omega$ is nondegenerate when restricted to $\mathrm{E}_{\chi} \oplus \mathrm{E}_{-\chi}$ and defines an isomorphism between $\mathrm{E}_{\epsilon \chi}$ and $\left(\mathrm{E}_{-\epsilon \chi}\right)^{*}$. The Oseledec decomposition (Proposition 9) can therefore be written as

$$
\mathrm{TV}=\left(\underset{i=1}{\oplus} \mathrm{E}_{i}^{-}\right) \oplus \mathrm{E}^{0} \oplus\left(\underset{i=1}{\oplus} \mathrm{E}_{i}^{+}\right),
$$

where $\operatorname{dim} E_{i}^{+}=\operatorname{dim} E_{i}^{-}$and the Lyapunov exponents are

$$
-\chi_{s}<\cdots<-\chi_{1}<0<\chi_{1}<\cdots<\chi_{s}
$$

Here, $E_{i}^{\epsilon}, \epsilon \chi_{i}$ and $s$ are measurable, $\varphi$-invariant functions on $\mathrm{V}$.

Given $v \in \Lambda$, denote by $\chi_{\max }(\mathrm{v})$ the maximum Lyapunov exponent at $\mathrm{v}$ and by $\mathrm{E}_{\max }^{\epsilon}(\mathrm{v}), \epsilon=+$, or - , the subspace in the Oseledec decomposition associated with $\epsilon \chi_{\max }(\mathrm{v})$. Analogously, define $\chi_{\min }(\mathrm{v})$ - the minimum positive Lyapunov exponent at $\mathrm{v}$ - and the associated linear space $\mathrm{E}_{m i n}^{\epsilon}(\mathrm{v})$.

Proposition 12. Assume the distributions $E^{+}$and $E^{-}$are $C^{\infty}$. Consider a $\varphi$ invariant smooth affine connection $\nabla$ on $V$ such that $\nabla E^{\epsilon} \subset E^{\epsilon}$, for $\epsilon=+$ or-. Given 
$\tau \in C_{i n v}^{\infty}\left({ }^{r} E^{*}\right)$, define the set $\Lambda_{\max }$ of points $v \in \Lambda$ at which $\nabla_{\xi_{0}} \tau \neq 0$ for some $\xi_{0} \in E_{\max }^{\epsilon}(v)$ and some $\epsilon \in\{+,-\}$. Then, we have $\lambda\left(\Lambda_{\max }\right)=0$.

Proof. By the multilinearity of $\tau$, it suffices to prove the lemma for the restrictions of $\tau$ to $\checkmark=\mathrm{E}^{\epsilon_{1}} \oplus \cdots \oplus \mathrm{E}^{\epsilon_{r}}, \epsilon_{i} \in\{+,-\}$. As before let $\rho: \checkmark \rightarrow \mathrm{V}$ be the base point projection. We may, of course, suppose $\Lambda_{\max } \neq \emptyset$.

Let $v \in \Lambda_{\max }$ and $\xi=\left(\xi_{1}, \cdots, \xi_{r}\right) \in \rho^{-1}(\mathrm{v})$ be a point in $\checkmark$ such that $\left(\nabla_{\xi_{0}} \tau\right)(\xi) \neq 0$ for some $\xi_{0} \in \mathrm{E}_{\max }^{\epsilon}(\mathrm{v})$. By linearity we may assume that $\xi_{i} \in \mathrm{E}_{l_{i}}^{\epsilon_{i}}(\mathrm{v}), \mathrm{i}=1, \cdots, \mathrm{r}$.

According to Proposition 11,

$$
\epsilon \chi \max (v)+\sum_{i=1}^{r} \epsilon_{i} \chi_{l_{i}}(v)=0 .
$$

Let $\mathrm{X} \in \mathrm{T}_{\xi} \checkmark$ be any tangent vector to $\mathscr{}$ at $\xi . \mathrm{X}$ can be given as

$$
\mathrm{X}=\left.\frac{\mathrm{d}}{\mathrm{d} \mathrm{t}}\right|_{\mathrm{t}=0} \tilde{\xi}(\mathrm{t})
$$

where $\mathrm{t} \mapsto \widetilde{\xi}(\mathrm{t})=\left(\widetilde{\xi}_{1}(\mathrm{t}), \cdots, \widetilde{\xi}_{r}(\mathrm{t})\right)$ is a smooth curve in $q$ such that

$$
\widetilde{\xi}(0)=\xi=\left(\xi_{1}, \cdots, \xi_{r}\right)
$$

and $\left.\frac{\mathrm{d}}{\mathrm{dt}}\right|_{\mathrm{t}=0} \rho \circ \widetilde{\xi}=\xi_{0}$.

We claim that

$$
(\mathrm{D} \tau)_{\xi} \mathrm{X}=\omega\left(\xi_{0}, \xi\right) \neq 0
$$

Note that once the claim is established, Lemma 3 yields $\lambda\left(\Lambda_{\max }\right)=0$.

We have

$$
\begin{gathered}
(\mathrm{D} \tau)_{\xi} \mathrm{X}=\left.\frac{\mathrm{d}}{\mathrm{dt}}\right|_{\mathrm{t}=0} \tau\left(\widetilde{\xi}_{1}(\mathrm{t}), \cdots, \widetilde{\xi}_{r}(\mathrm{t})\right) \\
=\left(\nabla_{\xi_{0}} \tau\right)(\xi)+\sum_{i=1}^{r} \tau\left(\xi_{1}, \cdots, \frac{\left.\nabla \widetilde{\xi}_{i}(0), \cdots, \xi_{r}\right) .}{\mathrm{dt}}(0),\right.
\end{gathered}
$$

If for some $\mathrm{i} \tau\left(\xi_{1}, \cdots, \frac{\nabla \widetilde{\xi}_{i}}{\mathrm{dt}}(0), \cdots, \xi_{r}\right)$ is not zero, there will be $\mu \in \mathrm{E}_{j}^{\epsilon_{i}}(\mathrm{v})$ such that

$$
\tau\left(\xi_{1}, \cdots, \xi_{i-1}, \mu, \xi_{i+1}, \cdots, \xi_{r}\right) \neq 0 .
$$


Again by Proposition 11 we obtain

$$
\left(\sum_{i=1}^{r} \epsilon_{i} \chi_{l_{i}}(\mathrm{v})\right)-\epsilon_{i} \chi_{l_{i}}(\mathrm{v})+\epsilon_{i} \chi_{j}(\mathrm{v})=0
$$

Combining this equation with $(*)$ we obtain

$$
\chi_{\max }(\mathrm{v})=\left|\chi_{l_{i}}(\mathrm{v})-\chi_{j}(\mathrm{v})\right|,
$$

a contradiction, since $\chi_{l_{i}}$ and $\chi_{j}$ are positive.

In [FK1] we used essentially the same proof as the one above to show that if the Lyapunov exponents of $\varphi$ satisfy

$$
\chi_{\max }<2 \cdot \chi_{\min }
$$

almost everywhere, then for every $\tau \in \mathrm{C}_{i n v}^{\infty}\left(\stackrel{r}{\otimes} \mathrm{E}^{*}\right), \nabla \tau \equiv 0$. We give below a slight generalization of this fact.

Consider the following situation. Suppose the distributions $\mathrm{E}^{\epsilon}$, for $\epsilon=+$ or - , decompose into $\varphi$-invariant smooth distributions $\mathrm{L}_{i}^{\epsilon}$ :

$$
\mathrm{E}^{\epsilon}=\stackrel{\oplus=1}{\oplus}_{i}^{\epsilon} \text {. }
$$

Let $\nabla^{\prime}$ be an invariant affine connection on $\mathrm{V}$ that is smooth and adapted to that decomposition, i.e., $\nabla^{\prime} \mathrm{L}_{i}^{\epsilon} \subset \mathrm{L}_{i}^{\epsilon}$, for all $\mathrm{i}$ and $\epsilon$.

At $v \in \Lambda$, define:

$$
\begin{gathered}
\underline{\chi}_{i}(\mathrm{v})=\inf \left\{\chi(\mathrm{v}, \xi): \xi \in \mathrm{L}_{i}^{+}(\mathrm{v})\right\} \\
\bar{\chi}_{i}(\mathrm{v})=\sup \left\{\chi(\mathrm{v}, \xi): \xi \in \mathrm{L}_{i}^{+}(\mathrm{v})\right\}
\end{gathered}
$$

Proposition 13. Assume that $E^{\epsilon}=\oplus L_{i}^{\epsilon}$ as above and let $\nabla^{\prime}$ be an invariant $C^{\infty}$ affine connection adapted to that decomposition. Assume moreover that

$$
\bar{\chi}_{i}<\underline{\chi}_{i}+\chi_{\min }
$$

almost everywhere and for all $i$. Then if $\tau \in C_{i n v}^{\infty}\left(\stackrel{r}{\otimes} E^{*}\right)$, we have

$$
\nabla^{\prime} \tau \equiv 0 \text {. }
$$


Proof. Assume $\nabla^{\prime} \tau \neq 0$. Let $\mathrm{v} \in \Lambda$ be such that $\left(\nabla^{\prime} \tau\right)_{v} \neq 0$ and assume that the condition on the exponents holds (since $\nabla^{\prime}$ and $\tau$ are flow invariant and the flow is ergodic with respect to the smooth measure $\lambda$, the set of such $v$ has full measure).

For $v \in \Lambda \cap\left\{\mathrm{v}:\left(\nabla^{\prime} \tau\right)_{v} \neq 0\right\}$, we can find $\xi_{i} \in \mathrm{E}_{l_{i}}^{\epsilon_{i}}(\mathrm{v})$ such that

$$
\left(\nabla_{\xi_{0}}^{\prime} \tau\right)\left(\xi_{1}, \cdots, \xi_{r}\right) \neq 0
$$

so that

$$
\sum_{i=1}^{r} \epsilon_{i} \chi_{l_{i}}(v)=0
$$

Let $\widetilde{\xi}_{i} \in \mathrm{L}_{k_{i}}^{\epsilon_{i}}$ be a smooth vector field defined near $\mathrm{v}$ that extends $\xi_{i}$. If

$$
\tau\left(\xi_{1}, \cdots, \nabla_{\xi_{0}}^{\prime} \widetilde{\xi}_{i}, \cdots, \xi_{r}\right) \neq 0
$$

for some $i$, then (since $\nabla^{\prime} L_{k_{i}}^{\epsilon} \subset L_{k_{i}}^{\epsilon}$ )

$$
\left(\sum_{i=1}^{r} \epsilon_{i} \chi_{l_{i}}(\mathrm{v})\right)-\epsilon_{i} \chi_{l_{i}}(\mathrm{v})+\epsilon_{i} \chi(\mathrm{v})=0
$$

for some $\chi$ such that

$$
\left|\chi_{i_{i}}-\chi\right| \leq \bar{\chi}_{k_{i}}-\underline{\chi}_{k_{i}}
$$

Therefore

$$
\epsilon_{0} \chi_{l_{0}}-\epsilon_{i} \chi_{l_{i}}+\epsilon_{i} \chi=0
$$

so that

$$
\chi_{\min } \leq \chi_{l_{0}}=\left|\chi_{l_{i}}-\chi\right| \leq \bar{\chi}_{k_{i}}-\underline{\chi}_{k_{i}},
$$

a contradiction. It then follows that

$$
0 \neq\left(\nabla_{\xi_{0}}^{\prime} \tau\right)\left(\xi_{1}, \cdots, \xi_{r}\right)=\xi_{0} \tau\left(\widetilde{\xi}_{1}, \cdots, \widetilde{\xi}_{r}\right),
$$

for an arbitrary smooth extension $\widetilde{\xi}_{i} \in \mathrm{L}_{k_{i}}^{\epsilon_{i}}$ of $\xi_{i} \in \mathrm{E}_{l_{i}}^{\epsilon_{i}}(\mathrm{v})$.

Now consider the smooth vector bundle

$$
\rho: \vartheta=\mathrm{L}_{k_{1}}^{\epsilon_{1}} \oplus \cdots \oplus \mathrm{L}_{k_{r}}^{\epsilon_{r}} \rightarrow \mathrm{V}
$$

We have shown that for all $\mathrm{v} \in \mathcal{A}=\left\{\mathrm{v}:\left(\nabla^{\prime} \tau\right)_{v} \neq 0\right\} \cap \Lambda$, there exists $\xi_{0} \in \mathrm{T}_{v} \mathrm{~V}$ and $\xi=\left(\xi_{1}, \cdots, \xi_{r}\right)$ in $\rho^{-1}(v)$ satisfying the properties of Lemma 3. But $\mathcal{A}$ has full measure, 
which contradicts the conclusion of that lemma.

Invariant and Smooth Subbundles of $\mathbf{E}^{\epsilon}$. Next we want to show how, from the existence of smooth invariant tensor fields that are not parallel with respect to some invariant and smooth affine connection $\nabla$, one obtains invariant smooth distributions properly contained in $\mathrm{E}^{\epsilon}$, for $\epsilon=+$ or - .

Proposition 14. Assume the same hypothesis of Proposition 12. Let $\tau \in C_{i n v}^{\infty}\left(\stackrel{r}{\otimes} E^{*}\right)$ be such that $\nabla_{\xi_{0}} \tau \neq 0$ for some $\xi_{0} \in E^{\epsilon}(v), v \in V$, and $\epsilon \in\{+,-\}$. Consider

$$
K^{\epsilon}(v)=\left\{\eta \in E^{\epsilon}(v): \nabla_{\xi_{0}} \tau \equiv 0\right\}
$$

Then the following hold:

(a) $v \mapsto K^{\epsilon}(v)$ is flow invariant and for almost every $v \in V, E_{\max }^{\epsilon}(v) \subset K^{\epsilon}(v)$. Moreover there exists a nonempty invariant open set where $K^{\epsilon}$ is $C^{\infty}$.

(b) Define $\left(K^{\epsilon}\right)^{\perp}(v)=\left\{\xi \in E^{-\epsilon}(v): \Omega\left(\xi, K^{\epsilon}(v)\right)=0\right\}$. Then $L^{-\epsilon}=h \operatorname{span}\left(K^{\epsilon}\right)^{\perp}$ is an invariant $C^{\infty}$ distribution of linear subspaces of $E^{-\epsilon}$ defined everywhere on $V$ and such that $L^{-\epsilon} \neq 0, E^{-\epsilon}$. Moreover $L^{-\epsilon}$ is invariant under the holonomy transport along $W^{* 0}$.

(c) Assume the following special situation: $E^{\epsilon}$ decomposes into the direct sum of two $\varphi-$ invariant $C^{\infty}$ subbundles $E^{\epsilon}=L_{1}^{\epsilon} \oplus L_{2}^{\epsilon}$. Let us suppose that $L_{1}^{\epsilon}$ and $L_{2}^{-\epsilon}$ are skeworthogonal with respect to $\Omega$, i.e., $\Omega\left(L_{1}^{\epsilon}, L_{2}^{-\epsilon}\right)=0$, and that for almost every $v \in V, L_{2}^{\epsilon}$ is the factor of the Oseledec decomposition of $T V$ associated to the exponent $\epsilon \chi_{\max }$. Let $\nabla^{\prime}$ be an invariant $C^{\infty}$ affine connection adapted to that splitting. If $\nabla^{\prime} \tau \neq 0$ for some $\tau \in C_{i n v}^{\infty}\left({ }^{r} E^{*}\right)$, then $(a)$ and $(b)$ still hold after replacing $E^{\epsilon}$ by $L_{1}^{\epsilon}$. In other words, $L_{1}^{\epsilon}$ contains a nontrivial invariant sub-bundle, which is $C^{\infty}$.

Proof. $\mathrm{K}^{\epsilon}$ can be viewed as the solution set of a system of linear equations smoothly parametrized by $\mathrm{v} \in \mathrm{V}$. Since $\nabla \tau$ is flow invariant, there exists a nontrivial 
invariant open set $\mathcal{A}^{\prime} \subset V$ on which $\mathrm{K}^{\epsilon}$ defines a smooth distribution. By ergodicity of $\lambda$, $\mathcal{A}^{\prime}$ has full measure.

On the other hand, the set

$$
\mathcal{A}^{\prime \prime}=\left\{\mathrm{v} \in \mathrm{V}:\left(\nabla_{\xi_{0}} \tau\right)_{v} \neq 0 \text { for some } \xi_{0} \in \mathrm{E}^{\epsilon}(\mathrm{v})\right\}
$$

is, by hypothesis, non-empty and clearly invariant, hence it also has full measure.

Let $\Lambda_{\max }$ be as given in Proposition 12. For every $\mathrm{v} \in \mathrm{S}=\mathcal{A}^{\prime} \cap \mathcal{A}^{\prime \prime} \cap\left(\mathrm{V}-\Lambda_{\max }\right)$,

$$
\mathrm{E}_{\max }^{\epsilon}(\mathrm{v}) \subset \mathrm{K}^{\epsilon}(\mathrm{v}) \neq \mathrm{E}^{\epsilon}(\mathrm{v})
$$

(The first inclusion and the fact that $\mathrm{S}$ has full measure is due to Proposition 12 and the second proper inclusion holds since $v \in \mathcal{A}^{\prime \prime}$.) This proves (a).

For regular points we have the Oseledec decomposition (O), where $E_{r}^{\epsilon}=E_{\max }^{\epsilon}$.

Define

$$
F^{\epsilon}=\stackrel{r=1}{\oplus}_{i=1}^{-1} E_{i}^{\varepsilon}=\left(\mathrm{E}_{\max }^{-\epsilon}\right)^{\perp}
$$

Then

$$
\mathrm{v} \in \mathcal{A}^{\prime} \mapsto\left(\mathrm{K}^{\epsilon}\right)^{\perp}(\mathrm{v}) \subset \mathrm{E}^{-\epsilon}(\mathrm{v})
$$

is a smooth distribution such that

$$
0 \neq\left(K^{\epsilon}\right)^{\perp}(v) \subset F^{-\epsilon}(v)
$$

for all $v \in S$. Therefore (b) follows from Propositions 6 and 10 .

In order to show $(\mathrm{c})$, let $\xi_{i} \in \mathrm{E}_{l_{i}}^{\epsilon_{i}}(\mathrm{v})$, for $\mathrm{i}=1, \cdots, \mathrm{r}(\mathrm{v} \in \Lambda)$ be vectors associated to the exponents $\epsilon_{i} \chi_{i_{i}}(\mathrm{v})$ and let $\xi_{0} \in \mathrm{E}_{r-1}^{\epsilon_{0}}(\mathrm{v})$, associated to $\epsilon_{0} \chi_{r-1}(\mathrm{v})$, so that

$$
\left(\nabla_{\xi_{0}}^{\prime} \tau\right)\left(\xi_{1}, \cdots, \xi_{1}\right) \neq 0
$$

By Proposition 11

$$
\epsilon_{0} \chi_{r-1}(v)+\sum_{i=1}^{r} \epsilon_{i} \chi_{l_{i}}(v)=0
$$

Under our assumptions, 


$$
\mathrm{L}_{1}^{\epsilon}=\stackrel{\oplus}{\oplus=1}_{i=1}^{\epsilon} \mathrm{E}_{i}^{\epsilon}, \mathrm{L}_{2}^{\epsilon}=\mathrm{E}_{r}^{\epsilon}
$$

almost everywhere, so we can assume this to be the case for the point $\mathrm{v}$ in $(*)$. We also have for the above $\xi$ 's that $\xi_{i} \in \mathrm{L}_{k_{i}}^{\epsilon_{i}}(\mathrm{v})$, for $\mathrm{k}_{i} \in\{1,2\}$ and each i. Let $\widetilde{\xi}_{i}$ be a smooth vector field extending $\xi_{i}$ in a neighborhood of $v$ such that $\widetilde{\xi}_{i} \in \mathrm{L}_{k_{i}}^{\epsilon_{i}}$ for each $\mathrm{i}$. We claim that

$$
\tau_{v}\left(\xi_{1}, \cdots, \nabla_{\xi_{0}}^{\prime} \widetilde{\xi}_{i}, \cdots, \xi_{r}\right)=0
$$

for all $i$.

If that is not the case, and since $\nabla_{\xi_{0}}^{\prime} \widetilde{\xi}_{i} \in \mathrm{L}_{k_{i}}^{\epsilon_{i}}(\mathrm{v})$, there exists $\eta \in \mathrm{L}_{k_{i}}^{\epsilon_{i}}(\mathrm{v})$ associated to the exponent $\epsilon_{i} \chi(\mathrm{v})$ such that

$$
\tau_{v}\left(\xi_{1}, \cdots, \eta, \cdots, \xi_{r}\right) \neq 0
$$

Again by Proposition 11,

$$
\left(\sum_{i=1}^{r} \epsilon_{i} \chi_{l_{i}}(v)\right)-\epsilon_{i} \chi_{l_{i}}(\mathrm{v})+\epsilon_{i} \chi(\mathrm{v})=0 .
$$

Combining this identity with $(*)$ we obtain

$$
\chi_{r-1}(v)=\left|\chi_{l_{i}}(v)-\chi(v)\right| .
$$

If $k_{i}=1$, there would be a vector in $\mathbf{L}_{1}^{\epsilon_{i}}$ whose exponent (either $\chi_{l_{i}}(\mathrm{v})$ or $\chi(\mathrm{v})$ ) was greater than $\chi_{r-1}(\mathrm{v})$, an impossibility. If $\mathrm{k}_{i}=2, \chi_{l_{i}}(\mathrm{v})=\chi(\mathrm{v})$, hence $\chi_{r-1}(\mathrm{v})=0$, which is also impossible. Therefore the claim must hold and it follows that

$$
0 \neq\left(\nabla_{\xi_{0}}^{\prime} \tau\right)\left(\xi_{1}, \cdots, \xi_{r}\right)=\xi_{0} \tau\left(\widetilde{\xi}_{1}, \cdots, \widetilde{\xi}_{r}\right)
$$

Exactly as in the end of the proof of Proposition 13, we conclude that the set of $v \in \Lambda$ such that $\nabla_{\xi_{0}}^{\prime} \tau \neq 0$ for some $\xi_{0} \in \mathrm{E}_{r-1}^{\epsilon_{0}}(\mathrm{v})$ has measure zero. On the other hand, we already know that $\nabla_{L_{2}^{\epsilon}}^{\prime} \tau \equiv 0$ so that $\nabla_{L_{1}^{\epsilon}}^{\prime} \tau \neq 0$. Therefore we can proceed as in parts (a) and (b), after replacing $\mathrm{E}^{\epsilon}$ by $\mathrm{L}_{1}^{\epsilon}$ in the definition of $\mathrm{K}^{\epsilon}$.

The Proof of Theorem 1. The following topological fact will be crucial in the proof of Theorem 1 . 
Proposition 15 ([S] Theorem 27.18). The m-sphere does not admit a continuous field of $k$-planes if $m$ is even and $1 \leq k \leq m-1$, or if $m \equiv 1(\bmod 4)$ and $2 \leq k \leq m-2$.

We return now to the setting of geodesic flows on manifolds of negative curvature. Recall the invariant affine connection $\nabla$ defined in section III. As explained before, the Theorem will be established after we prove the following.

Proposition 16. Let $M$ be a closed $C^{\infty}$ Riemannian manifold of dimension greater than two. Assume the Anosov foliations of the geodesic flow $\varphi_{t}: V \rightarrow V$ on the unit tangent bundle $V$ of $M$ are $C^{\infty}$. Assume moreover either $(a)-4<K \leq-1$ or else (b) the dimension of $M$ is odd. Let $\tau \in C_{i n v}^{\infty}\left(\stackrel{r}{\otimes} E^{*}\right)$. Then we must have $\nabla \tau \equiv 0$.

Proof. Under the assumption (a), the proposition follows from Proposition 13 in the particular case of $\chi_{\max }<2 \cdot \chi_{\min }$. Note that this condition on the exponents follows from the curvature assumption, according to Proposition 1.

Assume now that $M$ has odd dimension $n$, so that the ideal boundary $B$ of $\tilde{M}$ is a smooth sphere of even dimension. In particular, it does not admit a nontrivial field of $k$-planes for $1 \leq k \leq n-2$, due to Proposition 15 .

On the other hand, if we had $\tau \in C_{i n v}^{\infty}\left(\stackrel{r}{\otimes} E^{*}\right)$ with $\nabla \tau \neq 0$, Proposition 14 would yield a nontrivial smooth field of $k$-planes denoted there by $\mathrm{L}^{-\epsilon}$, invariant under the holonomy transport along $\mathrm{W}^{\epsilon 0}$. Therefore we obtain a continuous field of k-planes on $\mathrm{B} \sim \widetilde{\mathrm{V}} / \mathrm{W}^{\epsilon 0}$. But this is a contradiction.

The reason why we could define the $k$-plane field on $B$ was that $L^{-\epsilon}$ is invariant under the holonomy transport along $\mathrm{W}^{\epsilon 0}$. However, as is shown next, this property of $\mathrm{L}^{-\epsilon}$ is not necessary to establish Proposition 16 (although we preferred the given proof for it exhibits a structure on $B$ that may exist for even $n$. We will elaborate on this 
point in the next section). The following easy lemma gives a simpler way of obtaining the contradiction in the proof of the above proposition.

Lemma 4. Let $v \in \widetilde{V} \mapsto L(v) \subset E^{\epsilon}(v)$ be a continuous distribution of $k$-dimensional planes defined everywhere on $\widetilde{V}$. Let $n$ be the dimension of $M$. Then $L$ projects onto a continuous field of $k$-planes on an $(n-1)$-dimensional sphere.

Proof. Let $\mathrm{S}$ be a closed submanifold of $\tilde{\mathrm{M}}$ diffeomorphic to a sphere of dimension $n-1$. Let $\pi: \widetilde{V} \rightarrow \widetilde{M}$ denote the base point map and for each $x \in S$ denote by $\nu(\mathrm{x})$ the, say, inward unit normal vector to $\mathrm{S}$ at $\mathrm{x}$. For each $\mathrm{v} \in \widetilde{\mathrm{V}},(\mathrm{D} \pi)_{v}: \mathrm{E}^{\epsilon}(\mathrm{v}) \rightarrow \mathrm{v}^{\perp}$ is a linear isomorphism, where $\mathrm{v}^{\perp}$ is the orthogonal complement to $\mathrm{v}$ in $\mathrm{T}_{x} \mathrm{M}, \mathrm{x}=\pi(\mathrm{v})$. Therefore

$$
\mathrm{x} \in \mathrm{S} \mapsto(\mathrm{D} \pi)_{\nu(x)} \mathrm{L}(\nu(\mathrm{x})) \subset \mathrm{T}_{x} \mathrm{~S}
$$

define the desired field of $k$-planes on $S$. 


\section{Appendix}

In this final section we collect some scattered results, which point to a possible extension of the previous discussion to the case of $\operatorname{dim} M \equiv 2(\bmod 4)$.

The Theorem proven in the previous sections is a special case of the following problem.

Conjecture. Let $M$ be a closed $C^{\infty}$ Riemannian manifold of negative sectional curvature and dimension greater than two. Assume the Anosov foliations of the geodesic flow $\varphi_{t}: V \rightarrow V$ on the unit tangent bundle $V$ of $M$ are $C^{\infty}$. Then the geodesic flow of $M$ is $C^{\infty}$-isomorphic to the geodesic flow on a closed locally symmetricRiemannian manifold of negative curvature.

In fact, it is believed that a much stronger statement should hold, namely that it is enough for the conclusion of the conjecture to assume $\mathrm{C}^{2}$ foliations and, moreover, in the conclusion the manifold $M$ would be isometric to a Riemannian locally symmetric space of negative curvature. It is even expected that a similar rigidity phenomenon should hold for more general Anosov flows (and diffeomorphisms; see [FlK]) than geodesic flows on manifolds of negative curvature.

The natural next step after the Theorem proven here (which establishes the conjecture for $\operatorname{dim} M \equiv 1$ or $3(\bmod 4))$, is to consider the case $\operatorname{dim} M \equiv 2(\bmod 4)$. In these dimensions, a Riemannian locally-symmetric space of negative curvature is locally isometric to either $\mathbb{R H}^{2 m}$ or $\mathrm{CH}^{m}$ only.

If the invariant affine structure given by the Kanai connection $\nabla$ is locally symmetric, we already know from [K] that the conclusion of the conjecture holds. Therefore, in the following discussion, we assume $\nabla R \neq 0$ and that $\operatorname{dim} M \equiv 2(\bmod 4)$. 
Note that $\operatorname{dim} B \equiv 1(\bmod 4)$. Hence Propositions 14 and 15 imply the existence of smooth subbundles $E_{1}^{\epsilon}$ and $E_{2}^{\epsilon}$ of $E^{\epsilon}$, for $\epsilon=+$ and - , which are defined everywhere on $\mathrm{V}$ and

(1) $E_{1}^{\epsilon}$ is invariant under the holonomy transport along $W^{-\epsilon 0}$

(2) $\mathrm{E}_{2}^{\epsilon}=\left(\mathrm{E}_{1}^{-\epsilon}\right)^{\perp}=\Omega$-skew complement of $\mathrm{E}_{1}^{-\epsilon}$ in $\mathrm{E}$ and $\mathrm{E}_{\max }^{\epsilon} \subset \mathrm{E}_{2}^{\epsilon}$

(3) $\operatorname{dim} \mathrm{E}_{i}^{\epsilon}=1$, for $\mathrm{i}=1$ or 2 and $(\mathrm{DJ})_{v} \mathrm{E}_{i}^{\epsilon}(\mathrm{v})=\mathrm{E}_{i}^{-\epsilon}(\mathrm{J}(\mathrm{v}))$.

(4) On an open invariant set $\mathcal{A}$ (of full measure) $E_{1}^{\epsilon}$ and $E_{2}^{\epsilon}$ are transverse, i.e., $\mathrm{E}^{\epsilon}=\mathrm{E}_{1}^{\epsilon} \oplus \mathrm{E}_{2}^{\epsilon}$ on $\mathcal{A}$.

It is not clear, however, whether the decomposition in (4) holds everywhere on V. If this can be shown to be the case, then more is true. First, it would be possible to define another invariant affine connection $\nabla^{\prime}$ on $\mathrm{V}$ and its counterpart $\nabla^{\prime p}$ on $\mathrm{P}$, which are adapted to that finer splitting (we will give the details in the sequel).

Here are some consequences of assuming that (a) the decomposition in (4) holds on every point and (b) the sectional curvature of $M$ satisfies the following condition: $-9<\mathrm{K} \leq-1$.

Claim (1). $\mathrm{E}_{2}^{\epsilon}$ is a 1-dimensional smooth distribution, which coincides almost everywhere with $\mathrm{E}_{\max }^{\epsilon}$. Moreover, $\mathrm{E}_{1}^{\epsilon}$ is nonintegrable in the following strong sense. Let $\pi_{2}^{\epsilon}: \mathrm{TV} \rightarrow \mathrm{E}_{2}^{\epsilon}$ denote the natural projection and define a tensor field $\alpha \in \mathrm{C}_{i n v}^{\infty}\left(\mathrm{E}_{1}^{\epsilon *} \otimes \mathrm{E}_{1}^{\epsilon *} \otimes \mathrm{E}_{2}^{\epsilon}\right)$ such that for all $\mathrm{v} \in \mathrm{V}$ and all $\xi$ and $\eta \in \mathrm{E}_{1}^{\epsilon}(\mathrm{v})$

$$
\alpha(\xi, \eta)=\pi_{2}^{\epsilon}[\tilde{\xi}, \tilde{\eta}](\mathrm{v})
$$

where $\tilde{\xi}$ and $\tilde{\eta}$ are smooth vector fields in $\mathrm{E}_{1}^{\epsilon}$ extending $\xi$ and $\eta$ in a neighborhood of $\mathrm{v}$, which are, otherwise, arbitrary. Then, $\alpha$ is everywhere nonzero and, for every $\mathrm{v}$ in $\mathrm{V}$ we have: For all nonzero $\xi \in \mathrm{E}_{1}^{\epsilon}(\mathrm{v})$ there exists $\eta \in \mathrm{E}_{1}^{\epsilon}(\mathrm{v})$ such that $\alpha(\xi, \eta) \neq 0$. It follows that, for every positive Lyapunov exponent $\chi<\chi_{\max }$, there is another exponent $\chi^{\prime}$ such 
that $\chi+\chi^{\prime}=\chi_{\max }($ almost everywhere).

Claim (2). $\nabla^{\prime p}$ is a locally homogeneous affine connection, i.e., $\nabla^{\prime p} \mathrm{R}^{\prime p} \equiv 0$ and $\nabla^{\prime p} \mathrm{~T}^{\prime p} \equiv 0$ where $\mathrm{R}^{\prime p}$ and $\mathrm{T}^{\prime p}$ are the curvature and torsion of $\nabla^{\prime p}$. It is also a complete connection (this is due to an argument of L. Flaminio, which we do not reproduce here. See $[\mathrm{FlK}])$.

Claim (3). The group $G$ of affine transformations of $\nabla^{\prime p}$ preserving the symplectic form $\Omega$ and the distributions $\mathrm{F}_{i}^{\epsilon}$ on $\mathrm{P}$ acts transitively on $\mathrm{P}$ and its Lie algebra $\mathfrak{G}$ is a simple graded real Lie algebra of second kind (we will explain the terminology later). Disregarding the exceptional algebras, it follows from a theorem of Cheng $[\mathrm{C}]$ (see also $[\mathrm{KA}]$ ) that there are five families of possible candidates for $\mathfrak{B}$, among which is $\mathrm{su}(1, \mathrm{n})$ - the Lie algebra associated to the isometry group of the complex hyperbolic space.

In what follows, we would like to give the proofs of the claims stated above.

The Action of $\Gamma$ on $B$. The fundamental group $\pi_{1}(M)$ acts freely on $\tilde{M}$ as a group of isometries. Therefore it also acts on the unit tangent bundle $\tilde{V}$, permuting the orbits of $\varphi$ and the leaves of $W^{\epsilon 0}$. It follows that $\Gamma$ acts on $P$ and on $\tilde{V} / W^{\epsilon 0} \simeq B=$ the ideal boundary of $\tilde{M}$, as a group of homomorphisms. One property of this action on B is that for each element $\gamma \in \Gamma$ there are two distinct fixed points $b^{+}$and $b^{-} \in B$ such that under the iteration of $\gamma$ points of $\mathrm{B}$ other than $\mathrm{b}^{+}$or $\mathrm{b}^{-}$are attracted to $\mathrm{b}^{+}$and repelled by $\mathrm{b}^{-}$(see [EO]), i.e.,

$$
\bigcap_{n=1}^{\infty} \gamma^{n \epsilon}\left(B-\left\{b^{-\epsilon}\right\}\right)=\left\{b^{\epsilon}\right\}, \text { for } \epsilon=+ \text { and }- \text {. }
$$




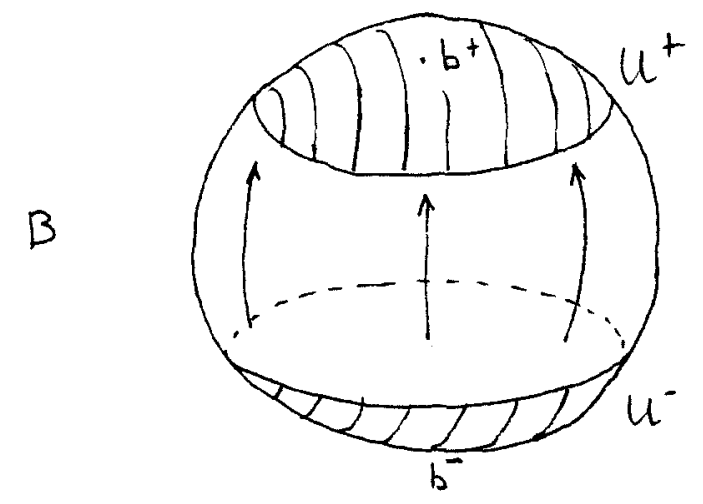

The following useful remark is due to M. Kanai. We thank him for explaining it to us.

Proposition 17. Let $B$ be a topological manifold of dimension $m$. Assume that $\gamma: B \rightarrow B$ is a homeomorphism with two fixed points $b^{+}$and $b^{-}$such that

$$
\bigcap_{n=1}^{\infty} \gamma^{n \epsilon}\left(B-\left\{b^{-\epsilon}\right\}\right)=\left\{b^{\epsilon}\right\}, \text { for } \epsilon=+ \text { and }- \text {. }
$$

Then $B$ does not admit a continuous $\gamma$-invariant foliation of dimension $l$ such that $2 \leq l \leq m-1$

Proof. Assume that such a foliation exists and call it $\mathscr{F}$. Let $\mathcal{U}^{+}$and $\mathcal{U}^{-}$be small neighborhoods of $\mathrm{b}^{+}$and $\mathrm{b}^{-}$, which are foliated domains for $\mathscr{F}$ (i.e., they are domains of foliated charts for $\mathscr{F}$ ) and such that $\mathcal{U}^{+}$does not contain $b^{-}$. Since $\mathscr{F}$ is $\gamma$ invariant, $\gamma^{k}\left(\mathcal{U}^{\epsilon}\right), \epsilon=+$ and,$- k \in \mathbb{Z}$, also are foliated domains for $\mathcal{F}$.

Let $k$ be a large enough integer so that the boundary of $\gamma^{k}\left(\mathrm{Ql}^{-}\right)$is contained in $\mathrm{Q}^{+}$and consider a connected leaf $\mathcal{L}^{-}$of $\mathcal{F}$ in $\gamma^{k}\left(\mathcal{L}^{-}\right)$, which does not contain $\mathrm{b}^{-}\left(\mathcal{L}^{-}\right.$is homeomorphic to a l-dimensional disc). The boundary of $L^{-}$is a connected set (since the dimension of the leaf is greater than 2) entirely contained in $\mathcal{U}^{+}$. Therefore this boundary is also the boundary of a connected leaf $\mathcal{L}^{+}$of $\mathscr{F}$ in $\mathcal{U}^{+}$(which is also 
homeomorphic to a l-dimensional disc). In this way, we have obtained a closed leaf of $f$ given by $\mathscr{L}=\mathcal{L}^{-} \cup \mathcal{L}^{+}$, which does not contain $\mathbf{b}^{-}$. By applying $\gamma$ several times to $\mathcal{L}$ we obtain a closed leaf of $\mathscr{F}$ contained in $\mathrm{U}^{+}$, a contradiction since $\mathrm{QL}^{+}$is a foliated domain of $\mathscr{F}$.

Corollary. There exists no foliation $\mathscr{F}$ of $V$ such that $F=T \mathscr{F} \subset E^{\epsilon}$ has dimension greater than 2 and $F$ is invariant under the holonomy transport along $W^{-\epsilon 0}$.

Proof. Such an $F$ would induce a $\Gamma$-invariant foliation on $B$.

Affine Connections Adapted to Smooth Decompositions of $\mathrm{E}^{\epsilon}$. Assume that $\mathrm{E}^{\epsilon}$ $(\epsilon=+$ or -$)$ decomposes as a direct sum of smooth $\varphi$-invariant subbundles $E_{i}^{\epsilon}, i=1, \cdots$, $\mathrm{k}$,

$$
\mathrm{E}^{\epsilon}=\mathrm{E}_{1}^{\epsilon} \oplus \cdots \oplus \mathrm{E}_{k}^{\epsilon}
$$

We assume that the decomposition is skew-orthogonal, i.e., $\Omega\left(\mathrm{E}_{i}^{\epsilon}, \mathrm{E}_{j}^{\delta}\right)=0$ if $\epsilon \neq \delta$ or $\mathrm{i} \neq \mathrm{j}$. Denote by

$$
\pi_{i}^{\epsilon}: \mathrm{TV} \rightarrow \mathrm{E}_{i}^{\epsilon}
$$

the linear projections and recall the invariant affine connection $\nabla\left(\nabla^{p}\right)$ on $\mathrm{V}(\mathrm{P})$ defined in section III. Define

$$
\nabla^{\prime}=\sum_{i, \epsilon} \pi_{i}^{\epsilon} \nabla \pi_{i}^{\epsilon}, \epsilon \in\{0,-,+\} \text { and } i \in\{1, \cdots, \mathrm{k}\} .
$$

Similarly we can consider $\nabla^{\prime p}$ on $\mathrm{P}$, adapted to the splitting of TP into the sub-bundles $F_{i}^{\epsilon}$, which are the projections of $E_{i}^{\epsilon}$ to $T P$.

Proposition 18. $\nabla^{\prime}\left(\nabla^{\prime p}\right)$ defines a $\varphi(\Gamma)$-invariant smooth affine connection on $V(P)$ such that (we omit the corresponding properties for $\nabla^{\prime p}$ )

$$
\begin{gathered}
\nabla^{\prime} E_{i}^{\epsilon} \subset E_{i}^{\epsilon}, \epsilon \in\{+,-, 0\} \text { and } i \in\{1, \cdots, k\} \\
\nabla^{\prime} \dot{\varphi} \equiv 0, \nabla^{\prime} \theta \equiv 0, \nabla^{\prime} d \theta \equiv 0 \\
\nabla_{\dot{\varphi}}^{\prime}=£_{\dot{\varphi}} .
\end{gathered}
$$


Proof. These are straighforward consequences of the properties of $\nabla$.

Lemma 5. Assume that $-9<K \leq-1$ and let $\tau \in C_{i n v}^{1}\left(\stackrel{3}{\otimes} E^{*}\right)$. Then, either $\tau \equiv 0$ or $\tau_{v} \neq 0$ for every $v \in V$.

Proof. Let $\|\cdot\|$ denote a Riemannian norm on TV. We use the same symbol $\|\cdot\|$ to denote the norm on the tensor algebra of TV. Suppose that $\tau \neq 0$ but there is a $v \in V$ where $\tau_{v}=0$. Let $\mathrm{u}$ be a point in $\mathrm{W}^{-}(\mathrm{v})$ such that $\tau_{u} \neq 0$. This is possible since $\mathrm{W}^{-}$is dense in V. Consider $\xi_{i} \in \mathrm{E}^{\epsilon_{i}}(\mathrm{u}), \epsilon_{i} \in\{+,-\}, \mathrm{i} \in\{1,2,3\}$, such that $\tau_{u}\left(\xi_{1}, \xi_{2}, \xi_{3}\right) \neq 0$. We can assume that the set $\left\{\epsilon_{1}, \epsilon_{2}, \epsilon_{3}\right\}$ contains more -'s than +'s (we could have considered $\mathrm{J}^{*} \tau$ instead of $\tau$, if that were not the case). Then, as

$$
\left\|\tau_{\varphi_{t}(u)}\right\| \leq \text { const } \cdot \mathrm{d}\left(\varphi_{t}(\mathrm{u}), \varphi_{t}(\mathrm{v})\right) \leq \mathrm{const} \cdot \mathrm{e}^{-t}, \quad 1 \leq(-\mathrm{K})^{1 / 2} \leq 3-\delta
$$

we have, for all positive $t$,

$$
\begin{aligned}
& 0<\left|\tau_{u}\left(\xi_{1}, \xi_{2}, \xi_{3}\right)\right|=\left|\left(\varphi_{t}^{*} \tau\right)_{u}\left(\xi_{1}, \xi_{2}, \xi_{3}\right)\right| \\
& =\left|\tau_{\varphi_{t}(u)}\left(\mathrm{D} \varphi_{t} \xi_{1}, \mathrm{D} \varphi_{t} \xi_{2}, \mathrm{D} \varphi_{t} \xi_{3}\right)\right| \\
& \leq\left\|\tau_{\varphi_{t}(u)}||\left|\mathrm{D} \varphi_{t} \xi_{1}\right|||\left|\mathrm{D} \varphi_{t} \xi_{2}\right| \mid\right\| \mathrm{D} \varphi_{t} \xi_{3} \| \\
& \leq \text { const } \cdot \mathrm{e}^{-t} \mathrm{e}^{-t} \mathrm{e}^{-t} \mathrm{e}^{(3-\delta) t} \\
& =\text { const } \cdot \mathrm{e}^{-\delta t} .
\end{aligned}
$$

But this is a contradiction.

We now proceed to proving the Claim (1). First, we show that $E_{2}^{\epsilon}$ is 1dimensional.

Let us suppose this is not the case, hence $E_{1}^{\epsilon}$ is 1-dimensional. Let $\pi_{1}^{-\epsilon}: T V \rightarrow E_{1}^{-\epsilon}$ denote the natural projection and consider the invariant tensor field $\tau \in \mathrm{C}_{i n v}^{\infty}\left(\stackrel{3}{\otimes} \mathrm{E}^{*}\right)$ on $\mathrm{V}$ given as follows:

$$
\tau=\nabla \beta, \text { where } \beta(\cdot, \cdot)=\Omega\left(\pi_{1}^{-\epsilon} \cdot, \cdot\right) .
$$

We have 
(*) If $\tau \neq 0$, then either $\tau\left(E_{1}^{\epsilon}, E_{2}^{-\epsilon}, E_{1}^{\epsilon}\right) \neq 0$ or $\tau\left(E_{1}^{\epsilon}, E_{1}^{\epsilon}, E_{2}^{-\epsilon}\right) \neq 0$ for $\epsilon=+$ or - .

Proof of $(*)$. Let $\mathrm{L}^{-\epsilon}$ be the distribution obtained in Proposition 14 (b). If the dimension of $\mathrm{L}^{-\epsilon}$ is greater or equal to 2 then, by Proposition 15 and Lemma $4, \mathrm{~L}^{-\epsilon}$ has in fact codimension 1 in $E^{-\epsilon}$ and $N^{\prime \epsilon}=\left(L^{-\epsilon}\right)^{\perp}$ - the (1-dimensional) skewcomplement of $\mathrm{L}^{-\epsilon}$ - coincides almost everywhere with $\mathrm{E}_{\max }^{\epsilon}$, which is almost everywhere contained in $\mathrm{E}_{2}^{\epsilon}$. Therefore $\mathrm{N}^{\prime \epsilon}$ is contained in $\mathrm{E}_{2}^{\epsilon}$. Again, by Lemma 4, we obtain a codimension 1 continuous distribution $N\left(\right.$ the projection of $E_{2}^{\epsilon}$ ) on a sphere of dimension $\mathrm{m} \equiv 1(\bmod 4)$ and, contained in that distribution, a continuous line field $\mathrm{N}^{\prime}$. But the orthogonal complement of $\mathrm{N}^{\prime}$ in $\mathrm{N}$ is a continuous distribution of codimension 2 on that sphere, contradicting Proposition 15. Therefore the dimension of $\mathrm{L}^{-\epsilon}$ is less than or equal to 1 .

If $\mathrm{L}^{-\epsilon}=0$, we have ( by the definition of $\mathrm{L}^{-\epsilon}$ ) that $\mathrm{K}^{\epsilon}=\mathrm{E}^{\epsilon}$, hence $\tau \equiv 0$, which is not the case. Otherwise, if the dimension of $\mathrm{L}^{-\epsilon}$ is 1 , then $\mathrm{K}^{\epsilon}$ has codimension 1 in $\mathrm{E}^{\epsilon}$. Now, if

$$
\tau\left(\mathrm{E}_{1}^{\epsilon}, \cdot, \cdot\right) \equiv 0
$$

we would have (by the definition of $K^{\epsilon}$ ) $E_{1}^{\epsilon} \subset K^{\epsilon}$. Again, this gives a 1-dimensional continuous distribution contained in a codimension 1 continuous distribution in $E^{\epsilon}$. But this was just shown to be impossible. Hence $\tau\left(E_{1}^{\epsilon}, \cdot, \cdot\right) \neq 0$.

It is clear, from $\Omega\left(E^{\epsilon}, E^{\epsilon}\right)=0$, that

$$
\tau\left(\cdot, \mathrm{E}^{\epsilon}, \mathrm{E}^{\epsilon}\right)=0
$$

so that $\epsilon_{2}=-\epsilon_{3}$. Also

$$
\tau\left(\cdot, \mathrm{E}_{2}^{-\epsilon}, \mathrm{E}_{2}^{\epsilon}\right)=0,
$$

since $\beta\left(\mathrm{E}_{2}^{\delta}, \cdot\right)=\beta\left(\cdot, \mathrm{E}_{2}^{\delta}\right)=0$ for $\delta=+$ or - , and

$$
\tau\left(\mathrm{E}, \mathrm{E}_{1}^{-\epsilon}, \mathrm{E}_{1}^{\epsilon}\right)=\mathrm{E} \beta\left(\mathrm{E}_{1}^{-\epsilon}, \mathrm{E}_{1}^{\epsilon}\right)-\beta\left(\nabla_{\mathrm{E}} \mathrm{E}_{1}^{-\epsilon}, \mathrm{E}_{1}^{\epsilon}\right)-\beta\left(\mathrm{E}_{1}^{-\epsilon}, \nabla_{\mathrm{E}} \mathrm{E}_{1}^{\epsilon}\right)
$$




$$
\begin{gathered}
=E \Omega\left(E_{1}^{-\epsilon}, E_{1}^{\epsilon}\right)-\Omega\left(\nabla_{E^{2}} E_{1}^{-\epsilon}, E_{1}^{\epsilon}\right)-\Omega\left(E_{1}^{-\epsilon}, \nabla_{E} E_{1}^{\epsilon}\right) \\
\left(\nabla_{E} \Omega\right)\left(E_{1}^{-\epsilon}, E_{1}^{\epsilon}\right)=0 .
\end{gathered}
$$

Hence

$$
\begin{gathered}
\tau\left(\mathrm{E}, \mathrm{E}_{i_{2}}^{-\epsilon}, \mathrm{E}_{i_{3}}^{\epsilon}\right)=0, \text { if } \mathrm{i}_{2}=\mathrm{i}_{3} \text { and } \epsilon=+ \text { and }- \\
\text { If } \mathrm{i}_{2}=2 \text { and } \epsilon_{1}=\epsilon_{2} \text {, we have } \\
\begin{aligned}
\tau\left(\mathrm{E}^{\epsilon}, \mathrm{E}_{2}^{\epsilon}, \mathrm{E}_{1}^{-\epsilon}\right)=\mathrm{E}^{\epsilon} \beta\left(\mathrm{E}_{2}^{\epsilon}, \mathrm{E}_{1}^{-\epsilon}\right)-\beta\left(\nabla_{\mathrm{E}^{\epsilon}} \mathrm{E}_{2}^{\epsilon}, \mathrm{E}_{1}^{-\epsilon}\right)-\beta\left(\mathrm{E}_{2}^{\epsilon}, \nabla_{\mathrm{E}^{\epsilon}} \mathrm{E}_{1}^{-\epsilon}\right) \\
=-\Omega\left(\nabla_{\mathrm{E}^{\epsilon}} \mathrm{E}_{2}^{\epsilon}, \mathrm{E}_{1}^{-\epsilon}\right) \\
=\Omega\left(\mathrm{E}_{2}^{\epsilon}, \nabla_{\mathrm{E}^{\epsilon}} \mathrm{E}_{1}^{-\epsilon}\right) \\
=0,
\end{aligned}
\end{gathered}
$$

where the last step is due to the fact that

$$
\nabla_{\mathrm{E}^{\epsilon}} \mathrm{E}_{1}^{-\epsilon} \subset \mathrm{E}_{1}^{-\epsilon}
$$

(Recall that $\mathrm{E}_{1}^{-\epsilon}$ is invariant under the holonomy transport along $W^{\epsilon 0}$ and that holonomy transport corresponds to parallel transport, according to Proposition 7.)

Analogously,

$$
\tau\left(\mathrm{E}^{\epsilon}, \mathrm{E}_{1}^{-\epsilon}, \mathrm{E}_{2}^{\epsilon}\right)=0
$$

Therefore we have $(*)$.

Next, we show that $\tau \neq 0$. Otherwise,

$$
\begin{gathered}
0=\tau\left(\mathrm{E}^{\epsilon}, \mathrm{E}_{2}^{-\epsilon}, \mathrm{E}_{1}^{\epsilon}\right) \\
=\mathrm{E}^{\epsilon} \beta\left(\mathrm{E}_{2}^{-\epsilon}, \mathrm{E}_{1}^{\epsilon}\right)-\beta\left(\nabla_{\mathrm{E}^{\epsilon}} \mathrm{E}_{2}^{-\epsilon}, \mathrm{E}_{1}^{\epsilon}\right)-\beta\left(\mathrm{E}_{2}^{-\epsilon}, \nabla_{\mathrm{E}^{\epsilon}} \mathrm{E}_{1}^{\epsilon}\right) \\
=-\Omega\left(\nabla_{\mathrm{E}^{\epsilon}} \mathrm{E}_{2}^{-\epsilon}, \mathrm{E}_{1}^{\epsilon}\right), \text { since } \Omega\left(\mathrm{E}_{2}^{-\epsilon}, \mathrm{E}_{1}^{\epsilon}\right)=0
\end{gathered}
$$

Hence $\nabla_{\mathrm{E}^{\epsilon}} \mathrm{E}_{2}^{-\epsilon} \subset \mathrm{E}_{2}^{-\epsilon}$, so that (according to Proposition 7) $\mathrm{E}_{2}^{-\epsilon}$ is invariant under the holonomy transport along $\mathrm{W}^{\epsilon 0}$. Moreover, since the same is true for $\mathrm{E}_{1}^{\epsilon}$, i.e.,

$$
\nabla_{\mathrm{E}^{-\epsilon}} \mathrm{E}_{1}^{\epsilon} \subset \mathrm{E}_{1}^{\epsilon}
$$

we have 


$$
\begin{aligned}
& 0=\Omega\left(\nabla_{\mathrm{E}^{-\epsilon}} \mathrm{E}_{1}^{\epsilon}, \mathrm{E}_{2}^{-\epsilon}\right) \\
& =-\Omega\left(\mathrm{E}_{1}^{\epsilon}, \nabla_{\mathrm{E}^{-\epsilon}} \mathrm{E}_{2}^{-\epsilon}\right),
\end{aligned}
$$

so that

$$
\nabla_{\mathrm{E}^{-\epsilon}} \mathrm{E}_{2}^{-\epsilon} \subset \mathrm{E}_{2}^{-\epsilon}
$$

But the torsion $\mathrm{T}$ of $\nabla$ has only component along the flow, so that

$$
\left[\mathrm{E}_{2}^{-\epsilon}, \mathrm{E}_{2}^{-\epsilon}\right] \subset \nabla_{\mathrm{E}_{2}^{-\epsilon}} \mathrm{E}_{2}^{-\epsilon} \subset \mathrm{E}_{2}^{-\epsilon} .
$$

Therefore $\mathrm{E}_{2}^{-\epsilon}$ is an integrable distribution. But this contradicts the Corollary to Proposition 18. Therefore $\tau \neq 0$.

According to what was just proven, at least one of the following holds

$$
\begin{aligned}
& \tau\left(\mathrm{E}_{1}^{\epsilon}, \mathrm{E}_{2}^{-\epsilon}, \mathrm{E}_{1}^{\epsilon}\right) \neq 0 \text { for } \epsilon=+ \text { or }- \\
& \tau\left(\mathrm{E}_{1}^{\epsilon}, \mathrm{E}_{1}^{\epsilon}, \mathrm{E}_{2}^{-\epsilon}\right) \neq 0 \text { for } \epsilon=+ \text { or }-
\end{aligned}
$$

The following analysis refers to case (1). The discussion for case (2) is totally analogous. Consider

$$
\tau^{\prime}(\cdot, \cdot, \cdot)=\tau\left(\pi_{1}^{\epsilon} \cdot, \pi_{2}^{-\epsilon} \cdot, \pi_{1}^{\epsilon} \cdot\right) .
$$

$\tau^{\prime}$ is a nonzero element of $\mathrm{C}_{i n v}^{\infty}\left(\stackrel{3}{\otimes} \mathrm{E}^{*}\right)$. By Lemma 4 and by what was proven above, $\tau^{\prime}$ is nowhere vanishing. Define, for $v \in V$

$$
\mathrm{N}(\mathrm{v})=\left\{\xi \in \mathrm{E}_{2}^{-\epsilon}: \tau^{\prime}\left(\mathrm{E}_{1}^{\epsilon}, \xi, \mathrm{E}_{1}^{\epsilon}\right)=0\right\} .
$$

Since $E_{1}^{\epsilon}$ is 1 -dimensional, $v \mapsto N(v)$ defines a codimension 1 distribution in $E_{2}^{-\epsilon}$, hence a codimension 2 distribution in $\mathrm{E}^{-\epsilon}$. Again, due to Lemma 4, this violates Proposition 15. This contradiction establishes that $E_{2}^{\epsilon}$ is 1-dimensional for $\epsilon=+$ and - and, in particular, $E_{2}^{\epsilon}=E_{\max }^{\epsilon}$ almost everywhere.

Now, we proceed to proving the nonintegrability property of $E_{1}^{\epsilon}$ claimed in (1). It is clear, from the fact that $\Omega\left(E_{1}^{\epsilon}, E_{2}^{-\epsilon}\right)=0$, that it will be sufficient to show the following distribution is identically zero: 


$$
L^{\epsilon}(v)=\left\{\xi \in E_{1}^{\epsilon}(v): \Omega_{v}\left(E_{2}^{-\epsilon},\left[\xi, E_{1}^{\epsilon}\right]\right)=0\right\}
$$

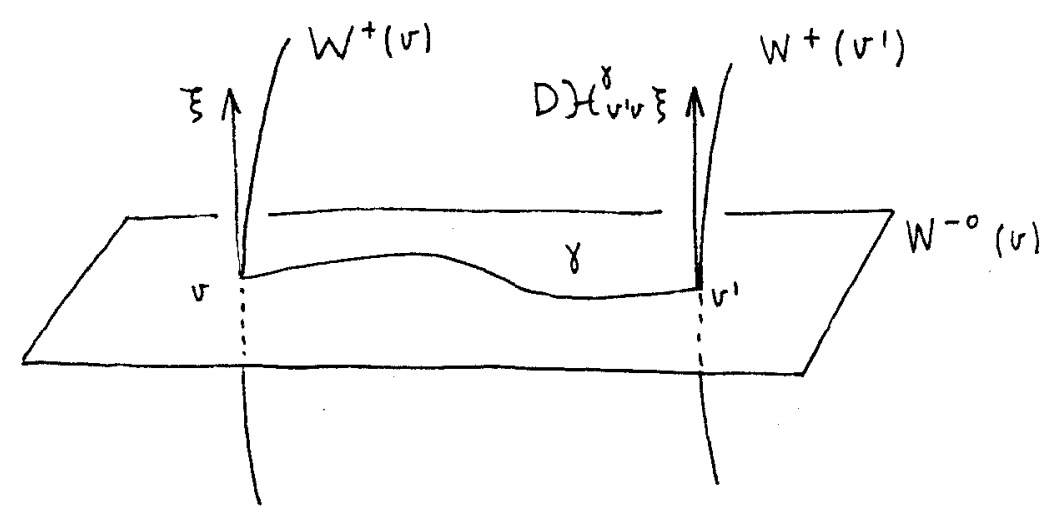

Recall the notation of section II (in the paragraph on holonomy). Let $\mathcal{U}$ be the domain of a foliated chart for $W^{\epsilon}$ and $W^{-\epsilon 0}$ and $v, v^{\prime}$ be any two points in $\mathcal{U}$ such that $v^{\prime} \in W_{q}^{-\epsilon 0}(v)$. Consider the holonomy map

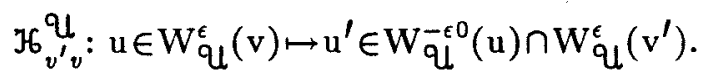

Since $\nVdash_{v^{\prime} v}^{\mathcal{U}}$ is a diffeomorphism, if $\xi$ and $\eta$ are vector fields on $\mathrm{W}_{\mathrm{U}^{\epsilon}}^{\epsilon}(\mathrm{v})$ (tangent to this leaf), we have

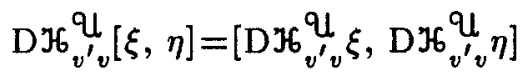

so that, if $\xi$ and $\eta$ are holonomy invariant (i.e., DJf ${ }_{v^{\prime}}^{q} \xi=\xi$, etc.) then so is $[\xi, \eta]$.

We already know that

$$
\mathrm{E}_{1}^{\epsilon} \circ J_{v^{\prime} v}^{\mathcal{U}}=\mathrm{D} J_{v^{\prime} v}^{\mathcal{U}} \mathrm{E}_{1}^{\epsilon} \text {. }
$$

Also note the following property of $\mathrm{E}_{2}^{-\epsilon}$. If $\gamma$ is any smooth curve in $\mathrm{W}_{\mathfrak{U}}^{-\epsilon 0}(\mathrm{u})$ joining the points $\mathrm{u}$ and $\mathrm{u}^{\prime}$, then $\mathrm{E}_{2}^{-\epsilon}$ is invariant under the parallel transport along $\gamma$, i.e.,

$$
\mathrm{P}^{\gamma} \mathrm{E}_{2}^{-\epsilon}=\mathrm{E}_{2}^{-\epsilon} \text {. }
$$

In fact, since $\Omega$ is a parallel tensor, we have 


$$
\begin{gathered}
\quad 0=\Omega_{u}\left(\mathrm{E}_{2}^{-\epsilon}(\mathrm{u}), \mathrm{E}_{1}^{\epsilon}(\mathrm{u})\right) \\
=\Omega_{u^{\prime}}\left(\mathrm{P}^{\gamma} \mathrm{E}_{2}^{-\epsilon}(\mathrm{u}), \mathrm{P}^{\gamma} \mathrm{E}_{1}^{\epsilon}(\mathrm{u})\right) \\
=\Omega_{u^{\prime}}\left(\mathrm{P}^{\gamma} \mathrm{E}_{2}^{-\epsilon}(\mathrm{u}), \mathrm{D}^{\jmath}{ }_{u^{\prime} u}^{\mathcal{u}} \mathrm{E}_{1}^{\epsilon}(\mathrm{u})\right) \\
=\Omega_{u^{\prime}}\left(\mathrm{P}^{\gamma} \mathrm{E}_{2}^{-\epsilon}(\mathrm{u}), \mathrm{E}_{1}^{\epsilon}\left(\mathrm{u}^{\prime}\right)\right)
\end{gathered}
$$

Therefore

$$
\mathrm{P}^{\gamma} \mathrm{E}_{2}^{-\epsilon}(\mathrm{u})=\text { the skew-complement of } \mathrm{E}_{1}^{\epsilon}\left(\mathrm{u}^{\prime}\right) \text { in } \mathrm{E}^{-\epsilon}\left(\mathrm{u}^{\prime}\right)=\mathrm{E}_{2}^{-\epsilon}\left(\mathrm{u}^{\prime}\right)
$$

Now, for $\mathrm{u}^{\prime}=36_{v^{\prime} v}^{\mathrm{q}}(\mathrm{u})$,

$$
\begin{gathered}
\mathrm{L}^{\epsilon}\left(\mathrm{u}^{\prime}\right)=\left\{\zeta \in \mathrm{E}_{1}^{\epsilon}\left(\mathrm{u}^{\prime}\right): \Omega_{u^{\prime}}\left(\mathrm{E}_{2}^{-\epsilon}\left(\mathrm{u}^{\prime}\right),\left[\tilde{\zeta}, \mathrm{E}_{1}^{\epsilon}\right]\left(\mathrm{u}^{\prime}\right)\right)=0\right\} \\
=\left\{\left(\mathrm{D} \xi_{v^{\prime} v}^{\mathrm{U}}\right){ }_{u} \zeta^{\prime}: \zeta^{\prime} \in \mathrm{E}_{1}^{\epsilon}(\mathrm{u}) \text { and } \Omega_{u^{\prime}}\left(\mathrm{E}_{2}^{-\epsilon}\left(\mathrm{u}^{\prime}\right),\left(\mathrm{D} \mathcal{F}_{v^{\prime} v}^{\mathrm{q}}\right)_{u}\left[\tilde{\zeta}^{\prime}, \mathrm{E}_{1}^{\epsilon}\right](\mathrm{u})\right)=0\right\} \\
=\left(\mathrm{D} \xi_{v^{\prime} v}^{q}\right)_{u}\left\{\zeta^{\prime} \in \mathrm{E}_{1}^{\epsilon}(\mathrm{u}): \Omega_{u^{\prime}}\left(\mathrm{P}^{\gamma} \mathrm{E}_{2}^{-\epsilon}(\mathrm{u}), \mathrm{P}^{\gamma}\left[\tilde{\zeta}^{\prime}, \mathrm{E}_{1}^{\epsilon}\right](\mathrm{u})\right)=0\right\}
\end{gathered}
$$

where $\gamma$ is any smooth curve in $W_{q}^{-\epsilon 0}(u)$ joining $u$ and $u^{\prime}$. Here, we are using the fact that $\mathrm{P}^{\gamma} \mu=\mathrm{D}^{\mathcal{H}}{ }_{v^{\prime} v^{\prime}} \mu$ for all $\mu \in \mathrm{E}^{\epsilon}$ and $\mathrm{P}^{\gamma} \mathrm{E}_{2}^{-\epsilon}=\mathrm{E}_{2}^{-\epsilon}$. But $\Omega$ is parallel, so

$$
\begin{aligned}
& L^{\epsilon}\left(u^{\prime}\right)=\left(D^{\prime} \mathcal{F}_{v^{\prime} v}^{q u}\right)_{u}\left\{\zeta^{\prime} \in E_{1}^{\epsilon}(u): \Omega_{u}\left(E_{2}^{-\epsilon}(u),\left[\tilde{\zeta}^{\prime}, E_{1}^{\epsilon}\right](u)\right)=0\right\} \\
& =\left(\mathrm{D} J \boldsymbol{G}_{v^{\prime} v}^{\mathrm{q}}\right)_{u} \mathrm{~L}^{\epsilon}(\mathbf{u}) \text {, }
\end{aligned}
$$

hence $L^{\epsilon}$ is invariant under the holonomy map.

It now follows from Proposition 6 that $L^{\epsilon}$ can be extended to a smooth distribution contained in $E_{1}^{\epsilon}$ and defined everywhere on $V$ (the extension being hspan $L^{\epsilon}$ ). Therefore by Proposition 15 and Lemma 4 , we must have $L^{\epsilon}=0$ or $E_{1}^{\epsilon}$. In the latter case (by the definition of $\mathrm{L}^{\epsilon}$ ), $\mathrm{E}_{1}^{\epsilon}$ would be integrable (in the usual sense). But this is impossible by the Corollary of Proposition 18. Therefore $L^{\epsilon}=0$.

The relation among the exponents, in Claim (1), follows now by applying Proposition 11 to the tensor field $\check{\alpha}=\Omega(\alpha(\cdot, \cdot), \cdot)$.

Now, we show that $\nabla^{\prime p}$ is a locally homogeneous connection. 
Let $\mathrm{R}^{\prime}$ and $\mathrm{T}^{\prime}$ denote the curvature and torsion of the $\varphi$-invariant connection $\nabla^{\prime}$, on V. Define

$$
\check{\mathrm{R}}^{\prime}=\Omega\left(\mathrm{R}^{\prime}(\cdot, \cdot) \cdot, \cdot\right) \text { and } \check{\mathrm{T}}^{\prime}=\Omega\left(\mathrm{T}^{\prime}(\cdot, \cdot) \cdot, \cdot\right) .
$$

It is not difficult to verify that $\check{\mathrm{R}}^{\prime} \in \mathrm{C}_{i n v}^{\infty}\left(\stackrel{4}{\otimes} \mathrm{E}^{*}\right)$ and $\check{\mathrm{T}}^{\prime} \in \mathrm{C}_{i n v}^{\infty}\left(\stackrel{3}{\otimes} \mathrm{E}^{*}\right)$ (the point here is to show that these tensors vanish when contracted with $\dot{\varphi}$ ).

From Proposition 14 (c) and the fact that $\mathrm{E}_{1}^{\epsilon}$ cannot contain a nontrivial continuous distribution of linear subspaces, it follows that

$$
\nabla^{\prime} \check{\mathrm{R}}^{\prime} \equiv 0 \text { and } \nabla^{\prime} \check{\mathrm{T}}^{\prime} \equiv 0 \text {. }
$$

But this implies that

$$
\nabla^{\prime p} \mathrm{R}^{\prime p} \equiv 0 \text { and } \nabla^{\prime p} \mathrm{~T}^{\prime p} \equiv 0
$$

A different way of establishing this fact is to use Proposition 13 and the following observation. Let $\chi_{1 \max }$ and $\chi_{\min }$ denote the maximum and minimum Lyapunov exponent for the restriction of $\mathrm{D} \varphi_{t}$ to $\mathrm{E}_{1}^{+}$. By Claim (1), we must have

$$
\chi_{\max }=\chi_{1 \max }+\chi_{\min }
$$

On the other hand, if $\chi_{1 \max } \geq 2 \cdot \chi_{\min }$, we would have $\chi_{\max } \geq 3 \cdot \chi_{\min }$, contradicting the assumption that $-9<\mathrm{K} \leq-1$. Therefore $\chi_{1 \max }<2 \cdot \chi_{\min }$, and we can apply Proposition 13.

The Group of Affine Transformations of $\nabla^{\prime p}$. Choose a point $x \in P$ and define

$$
\mathfrak{P}_{i}^{\epsilon}=\mathrm{F}_{i}^{\epsilon}(\mathrm{x}) \text {, for } \mathrm{i} \in\{1,2\} \text { and } \epsilon \in\{+,-\} \text {. }
$$

(Recall that $F_{i}^{\epsilon}$ is the projection of $E_{i}^{\epsilon}$ to TP.)

We regard a linear endomorphism $\alpha: \mathrm{T}_{x} \mathrm{P} \rightarrow \mathrm{T}_{x} \mathrm{P}$ as a derivation of the tensor algebra of $\mathrm{T}_{x} \mathrm{P}$. Hence, e.g.,

$$
\left(\alpha \cdot \mathrm{R}^{\prime p}\right)\left(\xi_{1}, \xi_{2}\right) \xi_{3}=\alpha \mathrm{R}^{\prime p}\left(\xi_{1}, \xi_{2}\right) \xi_{3}-\mathrm{R}^{\prime p}\left(\alpha \xi_{1}, \xi_{2}\right) \xi_{3}-\mathrm{R}^{\prime p}\left(\xi_{1}, \alpha \xi_{2}\right) \xi_{3}-\mathrm{R}^{\prime p}\left(\xi_{1}, \xi_{2}\right) \alpha \xi_{3} .
$$


Define

$$
\Re_{0}=\left\{\alpha \in \operatorname{End}\left(\mathrm{T}_{x} \mathrm{P}\right): \alpha \mathrm{F}_{i}^{\epsilon}(\mathrm{x}) \subset \mathrm{F}_{i}^{\epsilon}(\mathrm{x}), \alpha \cdot \mathrm{T}_{x}^{\prime p} \equiv 0, \alpha \cdot \mathrm{R}_{x}^{\prime p} \equiv 0, \alpha \cdot \Omega_{x} \equiv 0\right\}
$$

and

$$
\mathfrak{G}=\mathfrak{P}_{-2}+\mathfrak{B}_{-1}+\mathfrak{B}_{0}+\mathfrak{P}_{1}+\mathfrak{P}_{2} \text {. }
$$

Also define $\mathfrak{\beta}^{\epsilon}=\boldsymbol{P}_{\epsilon 2}+\mathfrak{P}_{\epsilon 1}$ and $\mathfrak{\beta}=\mathfrak{\beta}^{-}+\boldsymbol{\beta}^{+}$. The Lie brackets on $\mathcal{G}$ are defined as follows: For all $\xi, \eta \in \Re$ and $\alpha, \beta \in \Re_{0}$,

$$
\begin{gathered}
{[\alpha, \beta]=\alpha \circ \beta-\beta \circ \alpha} \\
{[\alpha, \xi]=\alpha \xi} \\
{[\xi, \eta]=-\mathrm{R}^{\prime p}(\xi, \eta)-\mathrm{T}^{\prime p}(\xi, \eta) .}
\end{gathered}
$$

G, equipped with these brackets, form a finite dimensional Lie algebra. This is an easy consequence of Bianchi's identities (see [KN]).

An essential feature of $\mathcal{G}$ is that it contains an element $\delta \in \mathbb{P}_{0}$ such that

$$
\left.\operatorname{ad}(\delta)\right|_{\Re_{i}}=\mathrm{iI}_{\Re_{i}}, \text { for } \mathrm{i} \in\{-2,-1,0,1,2\}
$$

Proof. Define $\delta \in \operatorname{End}\left(\mathrm{T}_{x} \mathrm{P}\right)$ by $(*)$. We need to show that $\delta \in \mathfrak{P}_{0}$. It is clear that $\delta \mathrm{F}_{i}^{\epsilon}(\mathrm{x}) \subset \mathrm{F}_{i}^{\epsilon}(\mathrm{x})$. Also

$$
\begin{gathered}
\left(\delta \Omega_{x}\right)\left(\mathrm{F}_{i}^{\epsilon}(\mathrm{x}), \mathrm{F}_{j}^{\epsilon^{\prime}}(\mathrm{x})\right)=\Omega_{x}\left(\delta \mathrm{F}_{i}^{\epsilon}(\mathrm{x}), \mathrm{F}_{j}^{\epsilon^{\prime}}(\mathrm{x})\right)+\Omega_{x}\left(\mathrm{~F}_{i}^{\epsilon}(\mathrm{x}), \delta \mathrm{F}_{j}^{\epsilon^{\prime}}(\mathrm{x})\right) \\
=\left(\epsilon \mathrm{i}+\epsilon^{\prime} \mathrm{j}\right) \Omega_{x}\left(\mathrm{~F}_{i}^{\epsilon}(\mathrm{x}), \mathrm{F}_{j}^{\epsilon^{\prime}}(\mathrm{x})\right) .
\end{gathered}
$$

But $\Omega_{x}\left(F_{i}^{\epsilon}(x), F_{j}^{\epsilon^{\prime}}(x)\right)=0$ unless $\mathrm{i}=\mathrm{j}$ and $\epsilon^{\prime}=-\epsilon$, in which case $\epsilon \mathrm{i}+\epsilon^{\prime} \mathrm{j}=0$, so that $\delta \cdot \Omega_{x}=\mathbf{0}$.

Now, consider $\delta \cdot \mathrm{T}^{\prime p}$. To show this is zero, suffices to prove that for all $\xi, \eta$ in $\mathrm{T}_{x} \mathrm{P}$

$$
\delta \mathrm{T}^{\prime p}(\xi, \eta)-\mathrm{T}^{\prime p}(\delta \xi, \eta)-\mathrm{T}^{\prime p}(\xi, \delta \eta)=0
$$

For that, it is clearly enough to show 


$$
\begin{gathered}
\mathrm{T}^{\prime p}\left(\mathrm{~F}_{1}^{\epsilon}(\mathrm{x}), \mathrm{F}_{2}^{\epsilon}(\mathrm{x})\right)=0, \mathrm{~T}^{\prime p}\left(\mathrm{~F}_{2}^{\epsilon}(\mathrm{x}), \mathrm{F}_{2}^{\epsilon}(\mathrm{x})\right)=0, \mathrm{~T}^{\prime p}\left(\mathrm{~F}_{i}^{-}(\mathrm{x}), \mathrm{F}_{i}^{+}(\mathrm{x})\right)=0 \\
\mathrm{~T}^{\prime p}\left(\mathrm{~F}_{1}^{\epsilon}(\mathrm{x}), \mathrm{F}_{1}^{\epsilon}(\mathrm{x})\right) \subset \mathrm{F}_{2}^{\epsilon}(\mathrm{x}), \mathrm{T}^{\prime p}\left(\mathrm{~F}_{1}^{-\epsilon}(\mathrm{x}), \mathrm{F}_{2}^{\epsilon}(\mathrm{x})\right) \subset \mathrm{F}_{1}^{\epsilon}(\mathrm{x})
\end{gathered}
$$

These relations, in turn, follow from the similar relations of $\mathrm{T}^{\prime}$, which are proven below. Let $v \in \tilde{V}$ be a pre-image of $x$.

$$
\begin{gathered}
\mathrm{T}^{\prime}\left(\mathrm{E}_{1}^{\epsilon}(\mathrm{v}), \mathrm{E}_{2}^{\epsilon}(\mathrm{v})\right)=0, \mathrm{~T}^{\prime}\left(\mathrm{E}_{2}^{\epsilon}(\mathrm{v}), \mathrm{E}_{2}^{\epsilon}(\mathrm{v})\right)=0, \mathrm{~T}^{\prime}\left(\mathrm{E}_{i}^{-}(\mathrm{v}), \mathrm{E}_{i}^{+}(\mathrm{v})\right) \subset \mathrm{E}^{0}(\mathrm{v}) \\
\mathrm{T}^{\prime}\left(\mathrm{E}_{1}^{\epsilon}(\mathrm{v}), \mathrm{E}_{1}^{\epsilon}(\mathrm{v})\right) \subset \mathrm{E}_{2}^{\epsilon}(\mathrm{v}), \mathrm{T}^{\prime}\left(\mathrm{E}_{1}^{-\epsilon}(\mathrm{v}), \mathrm{E}_{2}^{\epsilon}(\mathrm{v})\right) \subset \mathrm{E}_{1}^{\epsilon}(\mathrm{v}) .
\end{gathered}
$$

The proofs are all similar and we show only $\mathrm{T}^{\prime}\left(\mathrm{E}_{1}^{-}(\mathrm{v}), \mathrm{E}_{1}^{+}(\mathrm{v})\right) \subset \mathrm{E}^{0}(\mathrm{v})$, as an example. Define $\tau \in \mathrm{C}_{i n v}^{\infty}\left(\stackrel{3}{\otimes} \mathrm{E}^{*}\right)$ by

$$
\tau(\cdot, \cdot, \cdot)=\Omega\left(\mathrm{T}^{\prime}\left(\pi_{1}^{+} \cdot, \pi^{-} \cdot\right), \cdot\right),
$$

and, if $\tau \neq 0$, apply to it Proposition 11. There will be positive Lyapunov exponents $\chi<\chi_{\max }, \chi^{\prime}<\chi_{\max }, \chi^{\prime \prime}$ and $\epsilon \in\{+,-\}$ such that

$$
\chi-\chi^{\prime}+\epsilon \cdot \chi^{\prime \prime}=0 .
$$

If $\epsilon=+$, we have $\chi^{\prime}=\chi+\chi^{\prime \prime}$. If $\epsilon=-, \chi=\chi^{\prime}+\chi^{\prime \prime}$. There is no loss of generality in assuming that the latter case holds. From Claim (1), there exists a positive Lyapunov exponent $\chi^{\prime \prime \prime}$ such that

$$
\chi_{\max }=\chi+\chi^{\prime \prime \prime}=\chi^{\prime}+\chi^{\prime \prime}+\chi^{\prime \prime \prime} \geq 3 \cdot \chi_{\min } .
$$

But this is impossible, since the condition $-9<\mathrm{K} \leq-1$ implies $\chi_{\max }<3 \cdot \chi_{\min }$. Therefore $\tau \equiv 0$, as we wanted.

Now, we show $\delta \cdot \mathrm{R}^{\prime p} \equiv 0$. Since $\nabla^{\prime p} \mathrm{~F}_{i}^{\epsilon} \subset \mathrm{F}_{i}^{\epsilon}$, we have $\mathrm{R}^{\prime p}(\cdot, \cdot) \mathrm{F}_{i}^{\epsilon} \subset \mathrm{F}_{i}^{\epsilon}$, so that $\delta \circ \mathrm{R}^{\prime p}(\cdot, \cdot)=\mathrm{R}^{\prime p}(\cdot, \cdot) \circ \delta$. Hence, we only need to show that for all $\xi, \eta \in \mathrm{T}_{x} \mathrm{P}$

$$
\mathrm{R}^{\prime p}(\delta \xi, \eta)+\mathrm{R}^{\prime p}(\xi, \delta \eta)=0 .
$$

This property is a consequence of

$$
\mathrm{R}^{\prime}\left(\mathrm{E}_{i}^{\epsilon}, \mathrm{E}_{j}^{\epsilon^{\prime}}\right)=0 \text {, if } \mathrm{i} \neq \mathrm{i} \text { or } \epsilon^{\prime} \neq-\epsilon .
$$

We show only one instance of $(* *)$, say $\mathrm{R}^{\prime}\left(\mathrm{E}_{1}^{+}, \mathrm{E}_{2}^{-}\right) \mathrm{E}_{1}^{+}=0$, to illustrate the technique. 
Define, as we did for $\mathrm{T}^{\prime}$, the tensor field $\tau \in \mathrm{C}_{i n v}^{\infty}\left(\stackrel{4}{\otimes \mathrm{E}^{*}}\right)$ given by

$$
\tau(\cdot, \cdot, \cdot, \cdot)=\Omega\left(\mathrm{R}^{\prime}\left(\pi_{1}^{+} \cdot, \pi_{2}^{-} \cdot\right) \circ \pi_{1}^{+} \cdot, \pi_{2}^{-} \cdot\right) .
$$

If $\tau \neq 0$, Proposition 11 yields: there exist positive Lyapunov exponents $\chi, \chi^{\prime}, \chi^{\prime \prime}<\chi_{\max }$ such that

$$
\chi-\chi_{\max }+\chi^{\prime}-\chi^{\prime \prime}=0 .
$$

But by Claim (1), there exists Lyapunov exponents $\mu, \mu^{\prime}<\chi_{\max }$ such that

$$
\chi_{\max }=\chi+\mu=\chi^{\prime}+\mu^{\prime},
$$

so that

$$
\chi_{\max }=\chi^{\prime \prime}+\mu+\mu^{\prime} \geq 3 \cdot \chi_{\min } .
$$

This is a contradiction, proving $\tau \equiv 0$ and the existence of $\delta$.

Using the action of $\operatorname{ad}(\delta)$ on $\mathfrak{F}$, one readily obtains that $\mathfrak{P}^{\epsilon}, \epsilon=+$ and - , are nilpotent subalgebras of $\mathfrak{5}$ and

$$
\left[\Re_{i}, \Re_{j}\right] \subset \Re_{i+j} \quad\left(\Re_{k}=0 \text { if } \mathrm{k} \notin\{-2,-1,0,1,2\}\right),
$$

i.e., $\mathfrak{G}$ is a graded Lie algebra of 2 nd. kind.

Before continuing, we need to fix a few more definitions. From here on, we closely follow the steps of M. Kanai (see [K]).

Let $\mathrm{G}$ be a connected, analytic group with Lie algebra $\mathfrak{G}$. The element $\delta$ of $\mathfrak{G}$ defined above gives rise to the 1-parameter group of automorphisms $\phi$ of $\mathfrak{5}$

$$
\phi_{t}=\operatorname{Exp} t \cdot \operatorname{ad}(\delta): \mathfrak{G} \rightarrow \mathfrak{G},
$$

satisfying, for $\xi \in \Re_{j}, \mathrm{j} \in\{-2,-1,0,1,2\}$,

$$
\phi_{t} \xi=\mathrm{e}^{j \cdot t} \xi
$$

$\phi$ induces a 1-parameter group $\Phi$ of automorphisms of $G$ and the closed subgroup $H$ of $G$ consisting of fixed points of $\Phi$ is a Lie group with Lie algebra $\mathfrak{P}_{0}$.

Define the quotient $\hat{\mathrm{P}}=\mathrm{G} / \mathrm{H}$. Then $\hat{\mathrm{P}}$ is a reductive homogeneous G-space and 
we have the following facts. The proofs are analogous to the corresponding statements in $[\mathrm{K}]$ (one should pay attention to the fact that in $[\mathrm{K}] \hat{\mathrm{P}}$ is an affine symmetric space). (a) The subspaces $\aleph_{i}$, for $i \neq 0$, (which are $\operatorname{ad}\left(\$_{0}\right)$-invariant) define G-invariant distributions $\hat{\mathrm{F}}_{|j|}^{\epsilon}$ on $\hat{\mathrm{P}}$, where $\epsilon$ is the sign of $\mathrm{j} . \hat{\mathrm{F}}^{\epsilon}=\hat{\mathrm{F}}_{1}^{\epsilon} \oplus \hat{\mathrm{F}}_{2}^{\epsilon}$, for $\epsilon=+$ and - , are integrable distributions and we denote their foliations by $\hat{\boldsymbol{F}} \epsilon$.

(b) Identify $\Re \simeq \mathrm{T}_{0} \hat{\mathrm{P}}, \mathrm{o}=\mathrm{H} \in \mathrm{G} / \mathrm{H}$. Let $\hat{\nabla}$ denote the canonical $\mathrm{G}$-invariant connection on the reductive homogeneous space $\hat{\mathrm{P}}$ (see $[\mathrm{KN}]$ ). Let $\hat{\mathrm{T}}$ and $\hat{\mathrm{R}}$ be its torsion and curvature tensor. Then, at o we have: for $\xi, \eta, \zeta \in \Re$

$$
\begin{gathered}
\hat{\mathrm{T}}(\xi, \eta)_{o}=-[\xi, \eta]_{\Re}=\Re \text {-component of }-[\xi, \eta] \\
(\hat{\mathrm{R}}(\xi, \eta) \zeta)_{\circ}=-\left[[\xi, \eta]_{\mathfrak{\beta}_{0}}, \zeta\right] .
\end{gathered}
$$

Moreover $\hat{\nabla} \hat{T}=0, \quad \hat{\nabla} \hat{R}=0$ and $\hat{\nabla}$ is geodesically complete.

(c) The 1-parameter group of automorphisms $\Phi_{t}: G \rightarrow G$, (which commutes with the right multiplication by $\mathrm{H})$ descends to a 1-parameter group of automorphisms of $(\hat{\mathrm{P}}, \hat{\nabla}$, $\left.\hat{\mathscr{F}}^{+}, \hat{\mathfrak{F}}^{-}\right)$, which fixes $\mathrm{o}=\mathrm{H} \in \hat{\mathrm{P}} . \Phi_{t}$, for $\mathrm{t} \geq 0$, contracts the leaves of $\hat{\mathfrak{F}}^{-}$and expands the leaves of $\hat{\mathcal{F}}^{+} . \hat{\mathcal{F}}^{+}$and $\hat{\mathcal{F}}^{-}$are transverse, G-invariant foliations of $\hat{\mathrm{P}}$ of complementary dimensions.

(d) If $\mathcal{L}^{\epsilon}$ is a leaf of $\hat{\mathfrak{g}}^{\epsilon}$ for $\epsilon=+$ and - , then $\mathcal{L}^{+} \cap \mathcal{L}^{-}$contains at most one point ([K] Lemma 3.5)

(e) Define $\Re^{0 \epsilon}=\$_{0}+\$^{\epsilon}$ and let $\mathrm{H}^{\epsilon}$ denote the analytic subgroup of $\mathrm{G}$ with Lie algebra $\mathfrak{P}^{0 \epsilon}$. Then $\mathrm{H}^{\epsilon}, \epsilon=+$ and - , are closed subgroups of $\mathrm{G}([\mathrm{K}]$ Lemma 3.5). Therefore we can construct the homogeneous spaces $\hat{B}^{\epsilon}=G / H$.

(f) If $\mathfrak{U}$ is an ideal of $\mathfrak{G}$, then $\mathfrak{A}=\sum_{i} \mathfrak{A} \cap \mathfrak{P}_{i}, i \in\{-2,-1,0,1,2\}$ (similar to $[\mathrm{K}] 3.2$ ).

(g) If $\hat{\mathrm{B}}^{\epsilon}$ are compact, then $\mathfrak{G}$ is semisimple ([K], Lemma 3.6).

(h) There exists a developing mapping $\Psi: \mathrm{P} \rightarrow \hat{\mathrm{P}}$, which is an affine map with respect to the connections $\nabla^{\prime p}$ and $\hat{\nabla}$ and preserves the foliations (i.e., $\left.\Psi\left(\mathcal{F}^{\epsilon}(\mathrm{x})\right)=\hat{\sigma}^{\epsilon}(\Psi(\mathrm{x}))\right) . \Psi$ 
factors through $B^{\epsilon}$ and defines a covering map $\Psi^{\epsilon}: \hat{B}^{\epsilon}=\tilde{V} / W^{\epsilon 0} \rightarrow \hat{B} \quad(\epsilon=+$ and -$)$. Hence (since $\mathrm{B}^{\epsilon}$ is diffeomorphic to a sphere of dimension $\geq 2$ ), $\hat{\mathrm{B}}^{\epsilon}$ are compact. $\mathrm{By}(\mathrm{g})$ it follows that $\mathfrak{G}$ is semisimple (see [KN] vol $\mathrm{I}$, chapter IV and the discussion in [K] 4.1).

(i) $\mathfrak{G}$ is simple. (Kanai's proof of this fact does not seem to apply in our case, so we give a proof of it below).

Proof of (i). We already know that $\mathfrak{G}$ is semisimple. Let $\mathfrak{G}=\mathfrak{G}_{1} \oplus \cdots \oplus \mathfrak{G}_{k}$ be the decomposition of $\mathfrak{G}$ into its simple ideals. By (f), $\mathfrak{G}_{i}=\sum_{j} \mathfrak{G}_{i} \cap \Re_{j}$. Therefore, as $\mathfrak{P}_{2}^{+}$is 1 dimensional, there exists an i such that $\mathfrak{P}_{2}^{+} \subset \mathfrak{F}_{i}$. Let $\kappa$ denote the Killing form of $\mathfrak{F}(\kappa$ is nondegenerate).

By Lemma B.2 of $[C], \kappa\left(\Re_{2}, \beta_{-2}\right) \neq 0$. Hence

$$
0 \neq \kappa\left(\left[\delta, \Re_{2}\right], \Re_{-2}\right)=-\kappa\left(\delta,\left[\Re_{2}, \Re_{-2}\right]\right),
$$

so that $\left[\mathfrak{P}_{2}, \mathfrak{ß}_{-2}\right] \neq 0$. But $\mathfrak{\beta}_{-2} \subset \mathfrak{G}_{j}$ for some $j$ and $\left[\mathfrak{G}_{i}, \mathfrak{G}_{j}\right]=0$ unless $i=j$. Thus $\mathfrak{B}_{-2} \subset \mathfrak{G}_{i}$.

We claim that for all $0 \neq \xi \in \Re_{\epsilon 1}$, there exists $\eta \in \Re_{\epsilon 1}$ such that $[\xi, \eta] \in \mathbb{P}_{\epsilon 2}-\{0\}$ (we leave the proof of this fact to the end of the paragraph). Then for all $\xi \in \mathbb{P}_{1}-\{0\}$, there exists $\eta \in \Re_{1}$ such that

$$
0 \neq \kappa\left([\xi, \eta], \mathfrak{P}_{-2}\right)=-\kappa\left(\eta,\left[\xi, \mathfrak{P}_{-2}\right]\right) .
$$

Thus for all $\xi \in \mathbb{P}_{1}-\{0\},\left[\xi_{1}, \Re_{-2}\right] \neq 0$. Therefore the linear map

$$
\xi \in \Re_{1} \mapsto\left[\xi, \xi_{-2}\right] \in \Re_{-1}
$$

is an isomorphism, where $\xi_{-2}$ is a nonzero vector in $\mathfrak{P}_{-2}$ and we recall that $\mathfrak{P}_{1}$ and $\mathfrak{P}_{-1}$ have same dimension. But $\mathfrak{\beta}_{-2}$ is contained in the ideal $\mathfrak{G}_{i}$, so that $\mathfrak{\beta}_{-1}=\left[\mathfrak{P}_{1}, \mathfrak{\beta}_{-2}\right] \subset \mathfrak{G}_{i}$. Analogously, $\mathfrak{P}_{1} \subset \mathfrak{G}_{i}$, so that $\mathfrak{\beta \subset \mathfrak { G } _ { i }}$.

Also note that

$$
\left[\Re_{0} \cap \mathfrak{G}_{j}, \Re\right] \subset \mathfrak{G}_{j} \cap \Re \subset \mathfrak{G}_{j} \cap \mathfrak{G}_{i}=0 \text { if } i \neq j .
$$


But if $\alpha \in \Re_{0}$ is such that $[\alpha, \xi]=0$ for all $\xi \in \Re$, then $\alpha=0$ since by definition $[\alpha, \cdot]$ $\in \operatorname{End}(\mathfrak{P})$. Therefore $\mathfrak{P}_{0} \cap \mathfrak{G}_{j}=0$ for all $j \neq i$. It follows that

$$
\mathfrak{G}_{j}=\sum_{k} \mathfrak{P}_{k} \cap \mathfrak{G}_{j}=0 \text { if } \mathbf{j} \neq \mathrm{i}
$$

so that $\mathfrak{G}=\mathfrak{G}_{i}$.

It remains to show that

$$
\left[\xi, \mathfrak{P}_{\epsilon 1}\right]=\mathbb{P}_{\epsilon 2} \text { for all } 0 \neq \xi \in \mathbb{P}_{\epsilon 1} \text {. }
$$

Observe that

$$
\begin{aligned}
& {\left[\mathfrak{P}_{\epsilon 1}, \mathfrak{P}_{\epsilon 1}\right]=-\hat{\mathrm{R}}^{\prime p}\left(\mathfrak{P}_{\epsilon 1}, \mathfrak{P}_{\epsilon 1}\right)-\hat{\mathrm{T}}^{\prime p}\left(\mathfrak{P}_{\epsilon 1}, \mathfrak{P}_{\epsilon 1}\right)} \\
& =-\hat{\mathrm{T}}^{\prime p}\left(\Re_{\epsilon 1}, \Re_{\epsilon 1}\right)
\end{aligned}
$$

(recall that $\left.\hat{\mathrm{R}}^{\prime p}\left(\Re_{\epsilon 1}, \Re_{\epsilon 1}\right)=\mathrm{R}^{\prime p}\left(\mathrm{~F}_{1}^{\epsilon}(\mathrm{x}), \mathrm{F}_{1}^{\epsilon}(\mathrm{x})\right)=0\right)$. On the other hand, if $\xi, \eta \in F^{\epsilon 1}(\mathrm{x})$, and $\tilde{\xi}, \tilde{\eta}$ are extensions of $\xi$ and $\eta$ along $\mathrm{F}_{1}^{\epsilon}$, then

$$
\begin{aligned}
& \mathrm{T}^{\prime p}(\xi, \eta)=\nabla_{\xi}^{\prime p} \tilde{\eta}-\nabla_{\eta}^{\prime p} \tilde{\xi}-[\tilde{\xi}, \tilde{\eta}](\mathrm{x}) \\
&=\pi_{1}^{\epsilon} \nabla_{\xi}^{p} \tilde{\eta}-\pi_{1}^{\epsilon} \nabla_{\eta}^{p} \tilde{\xi}-\nabla_{\xi}^{p} \tilde{\eta}+\nabla_{\eta}^{p} \tilde{\xi} \\
&=\pi_{2}^{\epsilon} \nabla_{\xi}^{p} \tilde{\eta}-\pi_{2}^{\epsilon} \nabla_{\eta}^{p} \tilde{\xi} \\
&=\pi_{2}^{\epsilon}\left(\nabla_{\xi}^{p} \tilde{\eta}-\nabla_{\eta}^{p} \tilde{\xi}\right) \\
&=\pi_{2}^{\epsilon}[\tilde{\eta}, \tilde{\xi}](\mathrm{x}) \\
&=-\alpha(\xi, \eta) .
\end{aligned}
$$

Therefore (*) follows from Claim (1). 


\section{REFERENCES}

[A] D. V. Anosov, Geodesic Flows on Closed Riemann Manifolds with Negative Curvature. Proceedings of the Steklov Institute of Mathematics, n.90 (1967).

[C] J.-H. Cheng, Graded Lie Algebras of the Second Kind. Transactions of the American Mathematical Society, 302, n.2 (1987), 467-488.

[EO] P. Eberlein and B. O'Neill, Visibility Manifolds. Pacific J. Math. 46 (1973), 45109 .

[FK1] R. Feres and A. Katok, Invariant Tensor Fields of Dynamical Systems With Pinched Lyapunov Exponents and Rigidity of Geodesic Flows. To appear in Ergod. Th. Dynam. Systems

[FK2] R. Feres and A. Katok, Anosov Flows with Smooth Foliations and Rigidity of Geodesic Flows in Three-Dimensional Manifolds of Negative Curvature. Preprint.

[Fl-K] L. Flaminio and A. Katok , Rigidity of Symplectic Anosov Diffeomorphisms, Preprint.

[G] E. Ghys, Flots d'Anosov dont les Feuilletages Stables sont Differentiables. Preprint.

[H] B. Hasselblatt, Regularity of the Anosov Splitting and A New Description of the Margulis Measure. Ph.D. Thesis, California Institute of Technology (1989).

[He] S. Helgason, Differential Geometry, Lie Groups, and Symmetric Spaces. Academic Press, 1978.

[HK] S. Hurder and A. Katok, Differentiability, Rigidity and Godbillon-Vey Classes for Anosov Flows. Preprint.

[Ht] E. Heintze , On Homogeneous Manifolds of Negative Curvature. Math. Ann. 211, 23-34 (1974).

[K] M. Kanai , Geodesic Flows of Negatively Curved Manifolds with Smooth Stable and 
Unstable Foliations. Ergod. Th. Dynam. Systems, 8 , n. 2 (1988), p.215-240.

[KA] S. Kaneyuki and H. Asano, Graded Lie Algebras and Generalized Jordan Triple Systems. Preprint.

[Kl] W. Klingenberg , Riemannian Geometry, de Gruyter Studies in Mathematics, 1982.

[KN] S. Kobayashi and K. Nomizu , Foundations of Differential Geometry. v. I and II, John Wiley \& Sons, 1963.

[KNa] S. Kobayashi and T. Nagano, On Filtered Lie Algebras and Geometric Structures, I. J. Math. Mech. 13 (1964), 875-908; II, ibid. 14 (1965), 513-522.

[M] R. Mañe, Ergodic Theory and Differentiable Dynamics. Springer-Verlag, 1987.

[P] Ya. B. Pesin , Characteristic Lyapunov Exponents and Smooth Ergodic Theory, Russian Math. Surveys 32, 4 (1977), 55-114.

[S] N. Steenrod, The Topology of Fiber Bundles. Princeton University Press, 1951. 